This item was submitted to Loughborough's Research Repository by the author.

Items in Figshare are protected by copyright, with all rights reserved, unless otherwise indicated.

\title{
Inverse boundary spectral problem for Riemannian polyhedra
}

PLEASE CITE THE PUBLISHED VERSION

PUBLISHER

(C) Anna Kirpichnikova

\section{PUBLISHER STATEMENT}

This work is made available according to the conditions of the Creative Commons Attribution-NonCommercialNoDerivatives 4.0 International (CC BY-NC-ND 4.0) licence. Full details of this licence are available at: https://creativecommons.org/licenses/by-nc-nd/4.0/

\section{LICENCE}

CC BY-NC-ND 4.0

\section{REPOSITORY RECORD}

Kirpichnikova, Anna. 2019. "Inverse Boundary Spectral Problem for Riemannian Polyhedra". figshare. https://hdl.handle.net/2134/35982. 


\section{University Library}

\section{Loughborough \\ University}

Author/Filing Title ........KIRPICHNIKoVA....A.

Class Mark

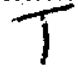

Please note that fines are charged on ALL overdue items.

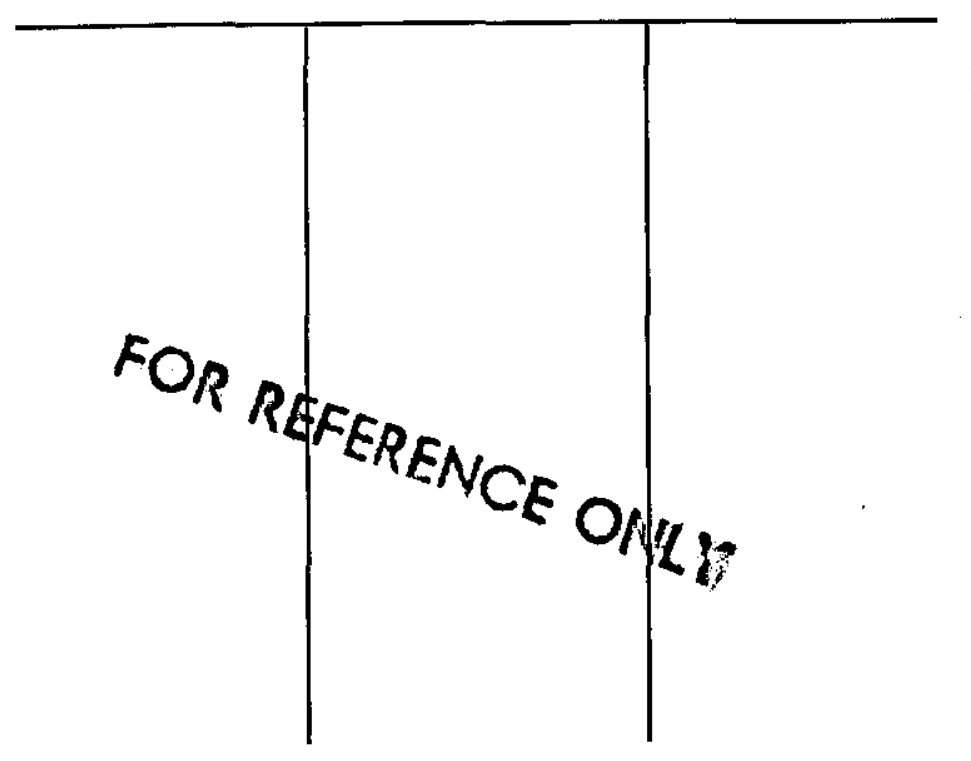




\section{Inverse Boundary Spectral Problem For Riemannian Polyhedra}
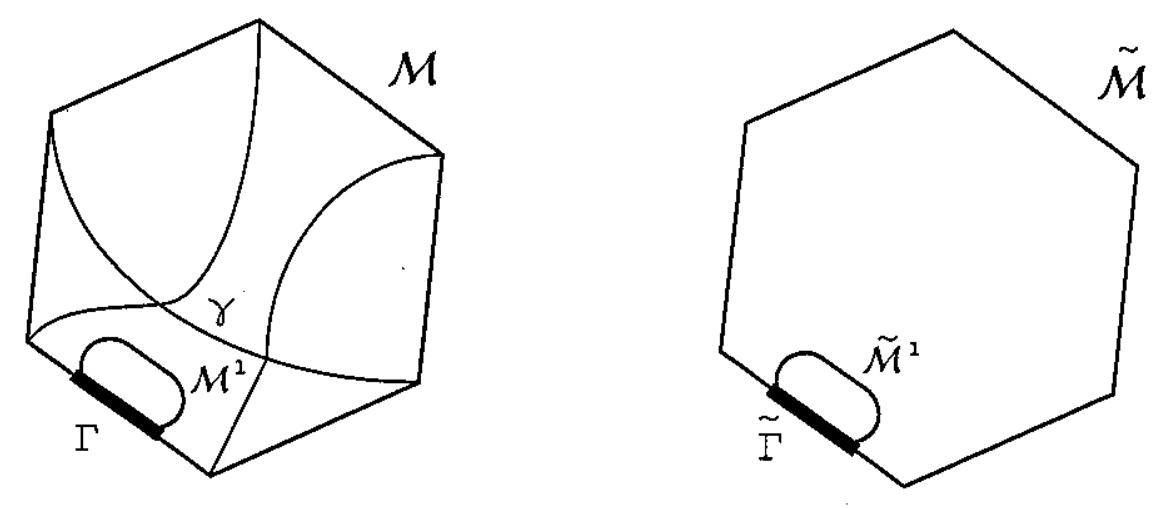

Anna Kirpichnikova

Department of Mathematical Sciences

Loughborough University - 2006 


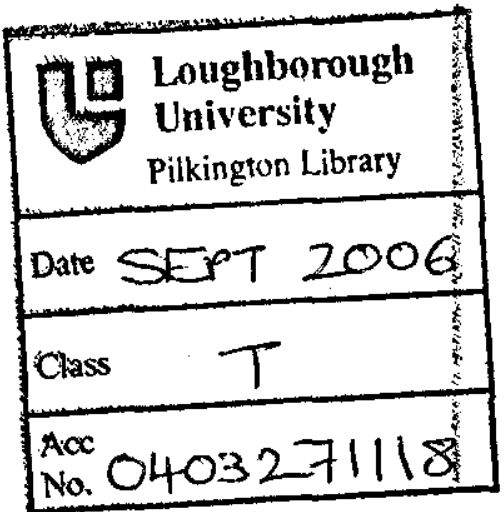




\title{
Inverse Boundary Spectral Problem for Riemannian Polyhedra
}

\author{
by
}

Anna Kirpichnikova

A Doctoral Thesis
Submitted in partial fulfillment of the requirements

for the award of Doctor of Philosophy in Mathematics of Loughborough University

March 2006

(C) by Anna Kirpichnikova 2006 


\section{Keywords:}

Piecewise smooth Riemannian metric

Riemannian Polyhedron

Inverse boundary spectral problem

Laplace-Beltrami operator

Gaussian beams
Abbreviations:
IBSP - inverse boundary spectral problem
IBVP - inverse boundary value problem
IP - inverse problem
BSD - boundary spectral data
BC-method - boundary control method
DBC - Dirichlet boundary conditions
ICC - interface continuity conditions
ARP - admissible Riemannian polyhedron 


\section{Abstract}

The object under consideration is an admissible Riemannian polyhedron $\mathcal{M}$ with a piece-wise smooth boundary $\partial \mathcal{M}$. This is a finite n-dimensional simplicial complex equipped with a family of Riemannian metrics smooth inside each simplex. We introduce an anisotropic Dirichlet Laplace operator in a weak sense for the admissible Riemannian polyhedron and define a set of boundary spectral data $\Gamma,\left\{\lambda_{k},\left.\partial_{\nu} \varphi_{k}\right|_{\Gamma}\right\}_{k=1}^{\infty}$ on a open part $\Gamma \subset \partial \mathcal{M}$, where $\lambda_{k}$ are the eigenvalues on $\Gamma$ and $\partial_{\nu} \varphi_{k} \mid \Gamma$ are the traces of normal derivatives of eigenfunctions of the Laplacian. The main result of the work is: if two admissible Riemannian polyhedra $\mathcal{M}$ and $\widetilde{\mathcal{M}}$ have open diffeomorphic parts of the boundaries $\Gamma \subset \mathcal{M}$ and $\tilde{\Gamma} \subset \widetilde{\mathcal{M}}$ such that the set of boundary spectral data on $\Gamma$ coincides with the set of boundary spectral data on $\tilde{\Gamma}$, then there is one-to-one correspondence between $\mathcal{M}$ and $\widetilde{\mathcal{M}}$ as simplicial complexes and they are also isometric as metric spaces. A new technique was developed to tackle the problem. That technique incorporated two methods: BC-method generalized and adjusted for the admissible Riemannian polyhedra and the technique of Gaussian beams extended for anisotropic piecewise smooth media. 


\section{Acknowledgements}

I would like to express my gratitude to the following people for their support and assistance during my work on this thesis:

My supervisor Prof. Yaroslav Kurylev, who provided me with excellent and enthusiastic supervision together with continuing motivation and encouragement. I could not have imagined having a better advisor and mentor for my $\mathrm{PhD}$. Thank you for all of the inspiration and the guidance you gave me during this work.

I also thank the following: The British Inverse Problems Society for giving me the chance to have several talks, and especially Prof. William Lionheart for his advise on the applications of the multi-component bodies. The Finnish Inverse Problems Society, especially Prof. Matti Lassas and Prof. Erkki Somersalo for their hospitality. Prof: Alexander Katchalov of the Steklov's Mathematical Institute, for the useful discussions on the asymptotical aspects of Gaussian beams.

All the rest of the academic and support staff of the Mathematical Sciences Department at Loughborough University for their kind assistance in many different ways. My research was financially supported by Loughborough University and ORS Award Scheme.

My parents and partner, for their understanding and encouragement when it was most required. 


\section{List of Figures}

2.1 Admissibility restrictions . . . . . . . . . . . . . . . 15

2.2 Two types of panels: interfaces and parts of the boundary . . . . . 15

2.3 Various notations of chambers . . . . . . . . . . . 20

3.1 Incident Gaussian beam and the interface . . . . . . . . . . . 39

3.2 Incidence, reflection, transmission Gaussian beams . . . . . . . . . . 44

3.3 Incidence, reflection, transmission coplanes and angles near the interface 59

4.1 We can reach any point on the manifold by path, which does not go into the collar neighborhood of the boundary. . . . . . . . . 88

5.1 Two admissible Riemannian polyhedra with equal BSD . . . . . . . 101

5.2 First subset identical on both polyhedra . . . . . . . . . . 102 
5.3 First chamber on both manifolds. We choose open domains inside first chambers. . . . . . . . . . . . . . . . . 103

5.4 Increasing subsets identical on both polyhedra . . . . . . . . . . 104

5.5 We assumed that the maximal subset is "less" than the first chamber. 105

5.6 Interface point on the second polyhedron. . . . . . . . . 106

5.7 Wedge point on the second polyhedron. . . . . . . . . . . 107

5.8 Various types of Gaussian beams reflections. . . . . . . . . . . 108

5.9 Crossing the interface on both polyhedra. . . . . . . . . . . . 110

5.10 Two different paths. . . . . . . . . . . . . . . . 112 


\section{Contents}

List of Figures

1 Introduction 1

1.1 Inverse problems $\ldots \ldots \ldots \ldots \ldots \ldots$

1.2 Background . . . . . . . . . . . . . . . . . 4

1.3 Outline of the thesis $\ldots \ldots \ldots \ldots \ldots$

2 Definitions and Geometric Description of the Problem 9

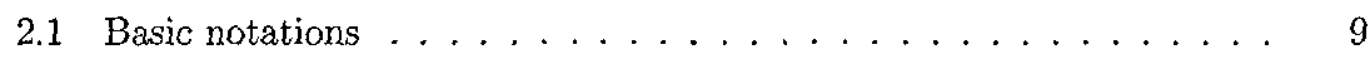

2.1 .1 Admissible polyhedron . . . . . . . . . . . . . . 12

2.2 Admissible Riemannian polyhedron . . . . . . . . . . . . . 17

2.2 .1 Metric structure . . . . . . . . . . . . 17 
2.2 .2 Coordinates . . . . . . . . . . . . . . 21

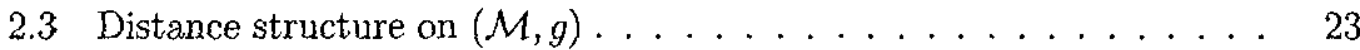

2.3 .1 Intrinsic distance $d_{X} \ldots \ldots \ldots 23$

2.4 Laplace-Beltrami operator . . . . . . . . . . . . . . . . . . . 31

2.5 Spectral problem $\ldots \ldots \ldots \ldots \ldots \ldots \ldots \ldots \ldots \ldots$

3 Gaussian Beams near the Interface 38

3.1. Gaussian beams - "quasiphotons". Definitions . . . . . . . . . . 38

3.1 .1 Solution form . . . . . . . . . . . . . 43

3.1 .2 Main results . . . . . . . . . . . . . . 45

3.1 .3 Formal series . . . . . . . . . . . . . . . 46

3.2 Phase functions $\ldots \ldots \ldots \ldots \ldots$

3.2 .1 Main equations . . . . . . . . . . . . . . . 47

3.2 .2 Required preparations $\ldots \ldots \ldots \ldots . \ldots . \ldots 50$

3.2.3 Impulses $\mathbf{p}^{r e f}, \mathrm{p}^{t r}$ construction $\ldots \ldots \ldots \ldots 1$

3.2 .4 Quadratic forms . . . . . . . . . . . . . . . . 53

3.2.5 Phase functions $\Theta_{N}^{r e f}(t, \mathbf{q}, \sigma), \Theta_{N}^{t r}(t, \mathbf{q}, \sigma) \ldots \ldots \ldots 57$

3.3 Reflection and transmission laws . . . . . . . . . . . 58 
3.4 Amplitudes . . . . . . . . . . . . . . . . . 62

3.4.1 Amplitude values on the interface ............ 62

3.4 .2 Amplitude equations . . . . . . . . . . . . . . 64

3.4 .3 Initial values $\ldots \ldots \ldots \ldots \ldots \ldots$

3.5 Exact and Approximate Solutions Estimates . . . . . . . . . . 66

3.5 .1 Convergence..................... 68

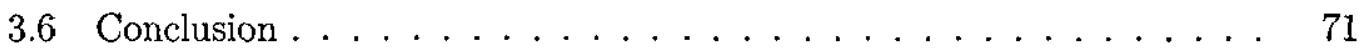

4 IBSP for a Smooth Riemannian Manifold 72

4.1 Reconstruction from BSD given on the boundary . . . . . . . 72

4.1.1 Formulation of the smooth problem . . . . . . . . 72

4.1.2 Reconstruction of the Fourier coefficients of the waves ....................... 73

4.1.3 Domains of influence. Complete system of functions.

Tataru's theorems. Wave basis.

Orthogonal projectors $\ldots \ldots \ldots \ldots \ldots$

4.1.4 On the role of Gaussian beams and boundary distance functions 80

4.2 IP with data given on a part of the boundary . . . . . . . . 84

4.2.1 First submanifold reconstruction . . . . . . . . . 84 
4.2.2 Recalculation of the boundary spectral data of $\left(\Delta_{\mathcal{D}}, \partial \mathcal{D}\right) \ldots 86$

4.2 .3 Reconstruction of $\mathcal{M}^{2} \ldots \ldots \ldots . \ldots . \ldots 87$

4.2.4 Iterating procedure; $\mathcal{M}^{m}=\mathcal{M}^{\text {int }} \ldots \ldots \ldots 88$

5 Uniqueness Problem for the Polyhedron 90

5.1 Formulation of the uniqueness problem . . . . . . . . . . 90 90

5.2 The Holmgren-John uniqueness theorem . . . . . . . . . . . 92

5.3 Uniqueness inverse problem . . . . . . . . . . . . . . . 101

5.4 Meeting the interface . . . . . . . . . . . . 105

5.5 Crossing the interface . . . . . . . . . . . . . . . 109

5.6 Polyhedra isometry . . . . . . . . . . . . . . 111

6 Conclusions and Outlook

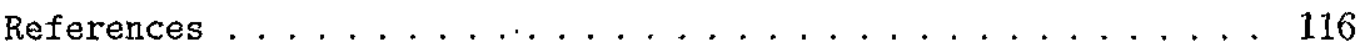




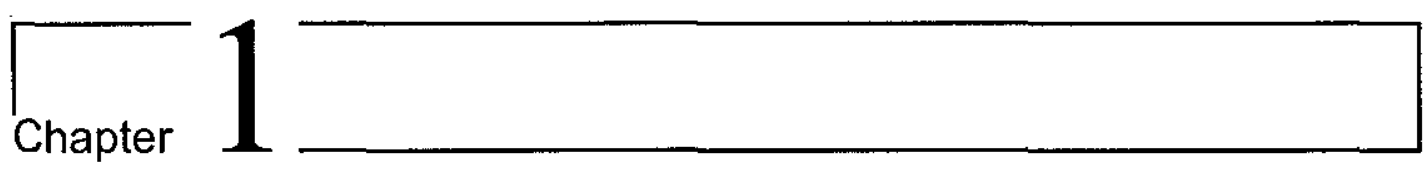

\section{Introduction}

\subsection{Inverse problems}

The general nature of an inverse problem is to deduce a cause from an effect. Consider a physical system, depending on a collection of parameters, in which one can speak of inputs to the system and outputs from the system. If all of the parameters were known perfectly then for a given input we could predict the output. It may happen, however, that some of the parameters characterizing the system are not known, being inaccessible to direct measurement. If it is important to know what these parameters are, in order that the system be understood as completely as possible, we might try to infer them by observing the outputs from the system corresponding to special inputs. Thus we seek the cause (the system parameters) given the effect (the output of the system for a given input). An important example is the inverse problem of geophysics, in which we seek to investigate the structure of the interior of the earth. Elastic waves may propagate through the earth in a manner which depends on the material properties of the earth. A concentrated source of energy at the earth surface causes waves to penetrate into the earth which are then partially reflected back to 
the surface. If the material properties of the earth's interior were known completely then we could predict the nature of the reflected wave from knowledge of the source. Since in fact we cannot measure these properties directly we seek to infer them by observing the reflected waves in response to a collection of known sources.

In formulating such problems mathematically, we typically find that the problem amounts to that of determining one or more coefficients in a differential equation, or system of differential equations, given partial knowledge of certain special solutions of the equation(s). In the seismology problem just discussed, the propagation of waves in the earth is governed by the equations of elasticity, a system of partial differential equations in which the material properties of the earth manifest themselves as coefficient functions in the equations. The measurements we can make amount to the knowledge of special solutions of the equations at special points, e.g. points on the surface of the earth in this example.

Inverse problems for differential equations have this general character. One has a certain definite kind of differential equation (or system of equations) containing one or more unknown (or partially known) coefficient functions. From some limited knowledge about certain special solutions of the equations we seek to determine the unknown coefficient functions. Problems of this type arise in a variety of important applications areas, such as geophysics, optics, quantum mechanics, astronomy, medical imaging and materials testing.

It is natural to consider problems with piece-wise smooth or even non-smooth coefficients (for instance the function of density has a jump), because the material is non-homogeneous. For example if we solve medical inverse problem, we consider bones, muscles, malesious tissue. If it is a geological problem, the earth is nonhomogeneous too: rocks, oils and so on. Mathematically we can consider the inverse problem with piece-wise smooth coefficients, i.e. functions with jumps. This goes out of the boundaries of the operators with smooth coefficients. The subject of this the- 
sis is an admissible anisotropic Riemannian polyhedron. This naturally arises from physics and real world structure - the multi-component body, each part (component) of which has its own geometrical and physical anisotropic properties. Mathematically these properties can be considered as an anisotropic metric structure and one can think about some differential operator given by this piece-wise smooth bounded metric. The problem is to determine the polyhedron structure, metric and the operator from given boundary spectral data. The problem formulated is of a great mathematical interest as it increases dramatically the class of functions for the inverse problems. This inverse problem has a great number of applications areas to be interested to as there are plenty of materials (for which we can measure only some boundary data) which have an anisotropic and multi-component structure.

Problems of this type may arise in a variety of important applications areas, such as geophysics, optics, quantum mechanics, astronomy, medical imaging and materials testing as anisotropy is widespread. An important example of such applications is the inverse problem of geophysics, in which one seeks to investigate the structure of the interior of the earth. The waves may propagate through the earth in a manner which depends on the material parameters of the earth. Consider other important examples of anisotropy applications: deformation, e.g. permittivity is anisotropic in a strained medium, and compressed soil can be anisotropic; crystalline or liquid crystal structure. Thus LC displays, biological thin films, colloids perhaps are the examples of considered structures together with fibrous or layered structures with anisotropic properties in the homogenization limit, layers of rock (thus the importance for geophysics), muscle (thus the importance for medicine), composite materials like fiberglass. Layers in the different density: air in the atmosphere, or water in the ocean. Alignment of particles in a flow, e.g. red blood cells of platelets of china clay in suspension are also such an example. The alignment of the particles gives rise to anisotropy in the homogenization limit. 


\subsection{Background}

Inverse spectral problem was firstly tackled by I. Gelfand, V. Marchenko, M. Krein, and B. Levitan in 1950's for the inverse problem of a string oscillations equation in a series of well-known publications (see e.g. [31], [44]). The method was based on reducing the problem to solving integral equation (see also [15]).

The method for reconstructing the density of non-homogeneous string in the multidimensional case appeared as boundary control method in the paper by M. Belishev [10]. M. Belishev considered both spectral and dynamical formulations of the problem. The possibility to control the system gives us an ability to obtain information about its structure due to the correspondence "control-respond". The boundary control method gives a procedure of density reconstruction for the bounded domain with a boundary. By its nature the boundary control method is a synthetical one. It uses asymptotical methods (discontinuities propagation, geometrical optics formulae), control theory, some elements of operator theory. As some inverse problems under consideration are over-determined one should assume that given data belong to some functional class, thus boundary control method uses the latest achievements of functional analysis.

Later the analytical ideas of boundary control method were combined with geometrical approach by M. Belishev, Ya. Kurylev [13], Ya. Kurylev [45]. The main idea of this approach was that any general elliptic second-order differential operator gives rise to a Riemannian metric in the corresponding domain, see also [9], [10], [12].

Some fundamental work on the inverse boundary spectral problem for a Riemannian manifold was obtained by A. Kachalov, Ya. Kurylev, M. Lassas (KKL) in 2001, see [38]. They produced a dynamical approach based on consideration of corresponding wave equations using various techniques to study an initial-boundary value problem for the anisotropic wave equation. 
The boundary control method developed by M. Belishev, A. Kachalov, Ya. Kurylev, M. Lassas for the case of a second-order differential operator in [38] works well for smooth coefficients. But when we try to consider the operator with non-smooth, or piece-wise smooth coefficients the method does not apply immediately. Geometrically piece-wise smooth manifolds can be described in terms of $n$ dimensional Riemannian polyhedrons. These polyhedrons can be joined by $(n-1)$-dimensional sub-manifolds, for instance wages, conic points. The anisotropic inverse problem has not been solved yet in the general case and it is very interesting and important to tackle it. Also in the case of multi-component body direct using of the boundary control method seems not to be possible, because of the complex geodesics behavior. For instance, if we have a cylinder, which consists of four regions with different velocity of wave propagation. These regions are formed by two lines. Assume that the velocity is similar and equal to one in two regions, corresponding to the vertical angles, formed by the lines. Let the velocity in the rest two regions is low, comparing to one. So, geodesics would try to go from the region with higher velocity to the other region with the same velocity without going through the low-velocity regions. So some geodesics will cohere in the vicinity of the intersection of the lines point. The function $\mathcal{R}(\mathcal{M})$ (it ascribe to each point on $\mathcal{M}$ the set of distances to the points of the boundary $\partial \mathcal{M}$ ) which is the main tool of boundary control method (see [38]) fails to be homeomorphic. To overcome these mathematical difficulties, the boundary control method should be essentially extended. Our idea here is to use boundary control method locally.

The complete solution to the unique continuation problem for the wave equation with time-analytic coefficients that involve a number of important new ideas was given by D. Tataru (see [56], [57], [58]).

The natural generalization of inverse boundary spectral problem to a multi-component body appeared in my investigations jointly with Ya. Kurylev. This work covers the proof of the uniqueness result for the pure (without potential) Laplace-Beltrami oper- 
ator of an admissible Riemannian polyhedra (the metric tensor is piece-wise smooth). The admissibility means that we have some restrictions on the type of simplicial complex, which is in the base of the admissible Riemannian polyhedron and that we have some restrictions on the smoothness class of the metric tensor corresponding to the polyhedron. This complicated object was investigated geometrically by J. Eells, B. Fuglede in 2001, see [25]. They introduced length space on it, they showed that there exists a shortest curve connecting two points on an admissible Riemannian polyhedron, such that it has no intersection with wedges and goes through the interfaces transversally.

I should also mention some investigations for the discontinuities of medium properties for the isotropic case. L. Päivärinta and K. Astala [4] reconstructed the conductivity operator when the conductivity is from $L^{\infty}$. Some smoother cases were done by $\mathrm{V}$. Isakov, G. Alessandrini, L. Päivärinta and A. Kirsch, see e.g. [2], [35], [42]. There are also some works on anisotropic conductivity, say paper by K. Astala, M. Lassas, L. Päivärinta [4]. 


\subsection{Outline of the thesis}

The purpose of this thesis is to prove the uniqueness theorem for the admissible Riemannian polyhedron determined by its boundary spectral data (BSD). Chapter 2 contains the definitions of the main subject, which is admissible Riemannian polyhedron. We refer to the book of J. Eells and B. Fuglede [25], (see also [29] and [30], H. Federer, [28]) to introduce all necessary information about its geometrical structure such as procedure of forming the length space. These procedures require accuracy as we cannot consider the admissible Riemannian polyhedron as a differentiable manifold, see the paper by M. Kervaire, [40]. In this Chapter we will sometimes use notations from papers of W. Ballman, [8]. This chapter also contains an important lemma from the paper by Ya. Kurylev, [45], stating that any two points on an admissible Riemannian polyhedron can be joined by a shortest curve which passes transversally the interfaces of the polyhedron in finite number of points and does not touch any other singular points of the polyhedron. We also refer to the books on differential geometry such as books by D. Burago, S. Burago, S. Ivanov, [17]; D. Burago, V. Zalgaller, [18]; A. Connel, [23].

The second part of Chapter 2 contains the information about the Laplace-Beltrami operator which can be introduced on the admissible Riemannian polyhedron in a weak sense, see the book of O. Ladyzhenskaya, [46], L. Evans, [27]. Chapter 3 contains the development of the Gaussian beams methods (see V. Babich, V. Ulin [6], V. Babich, V. Buldyrev, I. Molotkov, [7], A. Kachalov, [36], [37], M. Popov, [47], J. Ralston, [48]) to the case of the multidimensional anisotropic domain with an interface. The chapter contains all required definitions and the description of techniques used to tackle the problem. The necessary and sufficient for the next uniqueness problem asymptotics are found for the reflected from the interface field. It is shown that all approximate solutions are in the proper smoothness class and that for any small parameter $\varepsilon$ there exists an approximate solution to the wave equation which does not differ from the 
exact solution by more than some prescribed value. The results of Chapter 3 are published in [41].

Chapter 4 is totally descriptive. It contains a formulation of the smooth inverse problem for a smooth Riemannian manifold and brief description of the boundary control method taken from the book by A. Kachalov, Ya. Kurylev, M. Lassas, [38]. This Chapter covers the reconstruction procedure of the smooth Riemannian manifold $\mathcal{M}$ and a Laplace-Beltrami operator corresponding to it, i.e. reconstruction of the metric $g$ from a set of boundary spectral data (BSD) given on an open part of the boundary $\Gamma \subset \partial \mathcal{M}$. This Chapter also includes the required uniqueness theorems of Tataru's type (Local unique continuation result, Tataru's Theorem, the uniqueness Holmgren-John Theorem), see H. Koch, D. Tataru, [43], D. Tataru, [56], A. Kachalov, Ya. Kurylev, M. Lassas, [38], [5], Ya. Kurylev, [45].

The main part of this thesis is Chapter 5. This chapter contains the formulation and the proof of the inverse uniqueness theorem, which is the new and most valuable result of the thesis. Together with the main result Chapter 5 contains the proof of Holmgren-John uniqueness theorem for the piece-wise smooth structure, see [45]. 


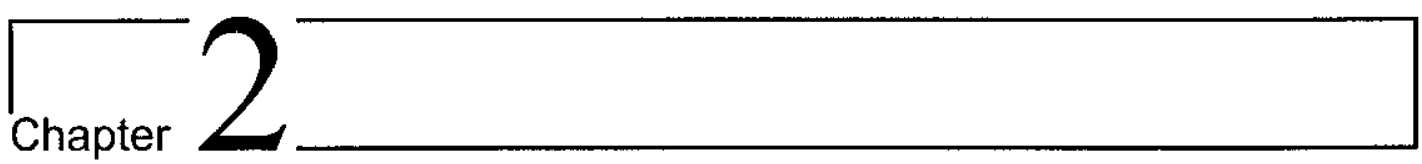

\title{
Definitions and Geometric Description of
}

\author{
the Problem
}

\subsection{Basic notations}

This section contains all basic definitions that will allow us to define the admissible polyhedron. We basically used the ideas of the book by J. Eells and B. Fuglede [25] and the well-known book on polyhedra by H. Seifert and W. Threlfall [51] to introduce this subject.

\section{Definition 2.1 (Length)}

Let $\left(Y, d_{Y}\right)$ be a metric space and $\eta: I=[a, b] \rightarrow Y$ a path. Its length is

$$
L(\eta)=\sup _{\pi}\left\{\sum_{i=1}^{r} d_{Y}\left(\eta\left(t_{i-1}\right), \eta\left(t_{i}\right)\right)\right\},
$$

where the supremum is taken over all partitions $\pi: a=t_{0}<\ldots<t_{r}=b$ of $I$. Say that $\eta$ is rectifiable if $L(\eta)<\infty$. 


\section{Definition 2.2 (Length space)}

A length space is a metric space $\left(Y, d_{Y}\right)$ such that for any pair of points $y_{0}, y_{1} \in Y$,

$$
d_{Y}\left(y_{0}, y_{1}\right)=\inf \left\{L(\eta): \eta \text { is a rectifiable path joining } y_{0} \text { to } y_{1}\right\}
$$

Such a metric is said to be intrinsic (or inner).

Every length space $\left(Y, d_{Y}\right)$ is locally connected. Indeed, every open ball $U=\{y \in$ $\left.Y: d_{Y}(a, y)<r\right\}$ is connected (even path connected). There is in fact a rectifiable path $\eta$ joining $a$ and $y \in U$ such that $L(\eta)<r$, and so $\eta$ has range in $U$ because, for any $z \in \eta$,

$$
d_{Y}(a, y) \leq d_{Y}(a, z)+d_{Y}(z, y) \leq L(\eta)<r .
$$

\section{Definition 2.3 (Geodesic space)}

A geodesic space is a length space $\left(Y, d_{Y}\right)$ for which any $y_{0}, y_{1} \in Y$ can be joined by a rectifiable path $\eta$ with $d_{Y}\left(y_{0}, y_{1}\right)=L(\eta)$.

\section{Definition 2.4 (Globally Lipschitz map)}

For metric spaces $\left(X, d_{X}\right)$ and $\left(Y, d_{Y}\right)$, class Lip $(X, Y)$ denotes the class of all globally Lipschitz maps $X \rightarrow Y$, i.e., maps $f$ for which there is a Lipschitz constant $c>0$ such that

$$
d_{Y}\left(f(x), f\left(x^{\prime}\right)\right) \leq c d_{X}\left(x, x^{\prime}\right), \quad x, x^{\prime} \in X .
$$

\section{Definition 2.5 (Lipschitz continuous)}

Map $f$ is said to be Lip continuous if it is locally Lipschitz. Lip loc $(X, Y)$ denotes the class of all Lipschitz continuous, i.e., locally Lipschitz, maps $f: X \rightarrow Y$. Thus every point of $X$ shall have a neighborhood $U$ such that $\left.f\right|_{U} \in \operatorname{Lip}(U, Y)$.

\section{Definition 2.6 (Lip homeomorphism)}

$f$ is said to be a Lip homeomorphism if $f$ is bijective and if $f$ and $f^{-1}$ are both Lip continuous. Similarly, $f$ is said to be Hölder continuous if $f$ satisfies a local Hölder condition. 
Remark 2.1.1 Every metric space $\left(X, d_{X}\right)$ is paracompact. This result can be found in [25].

\section{Definition 2.7 (Geodesic segment, Geodesic)}

$A$ (minimizing) geodesic segment of $\left(Y, d_{Y}\right)$, parameterized by path length, is a rectifiable path $\eta: I \rightarrow Y$ for which $d_{Y}(\eta(s), \eta(t))=|s-t|$ for all $s, t \in I$.

A geodesic of $\left(Y, d_{Y}\right)$ is a path $\eta: J \rightarrow Y$ whose restriction to every sufficiently small compact subinterval $I$ of $J=[0, a]$ is a geodesic segment. In other words, for any $x$ on $\eta$ there exists $I$ such that $x \in I$, and $\eta: J \cap I \rightarrow Y$ is a geodesic segment.

Statement 2.1.0.1 (Hopf-Rinow Theorem) For locally compact length space $Y$ the following conditions are equivalent:

1. Every half-open minimizing geodesic from a given point extends to a closed interval,

2. Every half-open geodesic extends to a closed interval,

3. Closed bounded subsets of $Y$ are compact,

4. $\left(Y, d_{Y}\right)$ is complete.

Any of these implies that $\left(Y, d_{Y}\right)$ is a geodesic space.

This theorem 2.1.0.1 is due to Cohn-Vossen, see e.g. [28]. 


\subsubsection{Admissible polyhedron}

Consider a finite simplicial complex $K$ of dimension $n$ see for example, [51], or, [25]. We recall that $K$ consists of a finite set $\{v\}$ of vertices, and a set $\{s\}$ of finite non-void subsets of vertices, called simplexes; $q$-simplex is a simplex with exactly $q+1$ vertices. Also

- any set consisting of exactly one vertex is a simplex.

- Any non-void subset of a simplex is a simplex.

Definition $2.8($ lin $K)$

A linear space of all formal finite linear combinations of vertices of $K$ is denoted by lin $K$.

\section{Definition 2.9 (Closed simplicial complex)}

We call complex $K$ closed (i.e. connected, homogeneous, finite) if the following two conditions hold:

- Every $(n-k)$-simplex of $K$ is contained in at least one $n$-simplex (dimensional homogeneity), $k=1,2, \ldots, n$.

- Every $(n-1)$-simplex of $K$ is adjacent to two $n$-simplices (in this case it is called interface boundary) or to one n-simplex and is then a part of the boundary $\partial K$.

Notation 2.1 ( $q$-skeleton of $K$ ) The $q$-skeleton $K^{q}$ of $K$ is the complex consisting of all its simplexes of dimension $\leq q$. We denote by $\mathcal{S}^{(q)}(K)$ the collection of all $q$-simplexes of $K$; and $\mathcal{S}(K):=\bigcup_{q} \mathcal{S}^{(q)}(K)$.

Definition 2.10 (The space $|K|$ of $K$; Barycentric coordinates)

The space $|K|$ of $K$ is the set of all finite linear combinations

$$
\alpha=\sum_{v \in K} \alpha(v) v
$$


of vertices of $K$ such that $0 \leq \alpha \leq 1, \sum_{v \in K} \alpha(v)=1$, and $\{v: \alpha(v)>0\}$ is a simplex of $K, \alpha(v)$ are barycentric coordinates of $\alpha \in|K|$.

Then $|K|$ is made into a metric space with barycentric distance $d(\alpha, \beta)$ between two points $\alpha=\sum \alpha(v) v$ and $\beta=\sum \beta(v) v$ of $|K|$ given by the finite sum

$$
d(\alpha, \beta)^{2}=\sum_{v \in K}(\alpha(v)-\beta(v))^{2}
$$

Then $K$ being finite, $|K|$ is separable and compact.

Remark 2.1.2 The space $|s|$ of a simplex $s$ of $K$ is convex as a subset of lin $K$.

We will from now on follow the notation from the work "Harmonic maps between Riemannian Polyhedra" by B. Fuglede and J. Eells [25], and partially the notations of W. Ballmann [8].

Notation 2.2 (Polyhedron) We shall use the term polyhedron to mean a connected compact separable Hausdorff space $X$ for which there exists a closed simplicial complex $K$ and a homeomorphism $\theta$ of $|K|$ onto $X$.

\section{Definition 2.11 (Lip Polyhedron)}

$X$ is called a (Lip) polyhedron when it is a connected compact separable Hausdorff space such that there exists a simplicial complex $K$ and a Lip homeomorphism $\theta$ of $|K|$ onto $X$, i.e. if a metric space $X$ is the image of metric space $|K|$ of some complex $K$ under a Lip homeomorphism $\theta:|K| \rightarrow X$.

\section{Definition 2.12 (Triangulation)}

Any pair $T=(K, \theta)$ is called a Lip triangulation of $K$.

We consider $K$ to be closed, $X$ to be compact, path connected and local contractible, the dimension of $X$ is equal to the dimension of $K$ (the dimension is independent on triangulation). The Lip Polyhedron $X$ has a metric corresponding via $\theta$ to the barycentric metric $d$ on $|K|$. 
Notation 2.3 (Vertices and Simplices of $X$ ) When $X$ is a polyhedron with a specified triangulation $T=(K, \theta)$, we shall speak of vertices of simplices of $(X, T)$ as the images under $\theta$ of vertices of simplices $|s|$ (we can simply write $s$ ) of $K$. Thus our "new" simplices become compact subsets of $X$. The interior of $k$-simplex $s$ of $(X, T)$ (i.e. the points of $s$ not in any $(k-1)$-simplex of $s$ ) is denoted by $\stackrel{\circ}{s}$. A 0 -simplex is called a vertex, in this case $\stackrel{\circ}{s}=s$.

Notation 2.4 (Set of all Wedge Points) Denote by $W P^{k}(X)=W P^{k}(X, T)$ the collection of $k$-simplices of $(X, T)$; by $W P(X)=W P(X, T)$ the collection of all simplices of $(X, T)$; by $\mathcal{W}(X)=\mathcal{W}(X, T)$ the collection of all $n$-simplices.

Notation 2.5 (Subpolyhedron) A subpolyhedron of a polyhedron $X$ with a specific triangulation $T=(K, \theta)$ is a polyhedron $X^{\prime} \subset X$ having as a triangulation $T^{\prime}=\left(K^{\prime},\left.\theta\right|_{\left|K^{\prime}\right|}\right)$ where $K^{\prime}$ is a subcomplex of $K$.

\section{Definition 2.13 ( $k$-skeleton)}

For $0 \leq k \leq \operatorname{dim} X$ the $k-$ skeleton $X^{k}$ of $X$ (the union of all simplices $|s|$ of dimension $\leq k$ ) is a sub-polyhedron of $X$, independent of the triangulation of $X$ and closed in $X$.

\section{Definition 2.14 (Admissible Polyhedron)}

Polyhedron $X$ is called admissible if in some (hence in any, see [25], see also Figure 2.1) triangulation

1. Polyhedron $X$ is dimensionally homogeneous, i.e. every $(n-k)$-simplex is contained in at least one n-simplex, see the left hand side of Figure 2.1, $k=$ $1,2, \ldots, n, n$ is a dimension of $X$. The $n$-simplices are called chambers.

2. The boundary $\partial X$ is a union of all $(n-k)$-simplices, $k=1,2, \ldots, n$ contained in only one chamber, $\partial X$ is a closed subset of $X$ and a subpolyhedron of $(X, T)$, independent of the triangulation of $X$. 

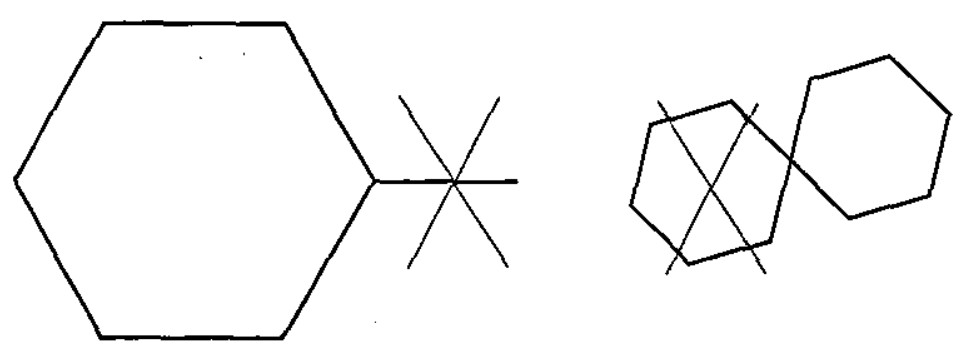

Figure 2.1: Admissibility restrictions
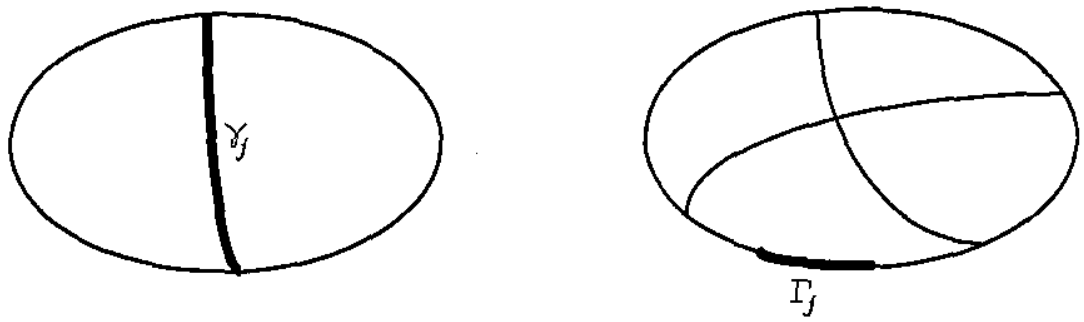

Figure 2.2: Two types of panels: interfaces and parts of the boundary

3. Every $(n-1)$-simplex of $X$ is called panel and is adjacent to either two chambers (and is called interface boundary in this case) or adjacent to only one chamber and then is a part of the boundary $\partial X$ (see Figure 2.2).

4. Polyhedron $X$ is $(n-1)$-chainable, i.e. any two chambers can be joined by a chain of continuous panels and chambers, see the right hand side of Figure 2.1.

\section{Definition 2.15 (Star of $X$, Carrier of a point)}

The (open) star of an open simplex $\stackrel{\circ}{s}$ of $X=(X, T)$ is defined as

$$
s t(\stackrel{\circ}{s})=s t_{X}(\stackrel{\circ}{s})=\bigcup\{\stackrel{\circ}{t}: t \in W P(X) \text { with } t \supset s\} .
$$

The star st(a) of a point $a \in X$ is defined as a star of its carrier, the unique open simplex $s$ containing a. Every star is a path connected open subset of $X$ and contains the star of its points. 


\section{Definition 2.16 (Singular Set)}

Denote by $\Sigma=\Sigma(X)$ the singular set of $X$, i.e. the complement of the set of all points of $X$ having a neighbourhood which is a topological $n$-manifold possibly with boundary.

We should notice that $\Sigma$ is a closed triangulable subspace of $X$ of codim $\geq 2$ and $X \backslash \Sigma$ is a topological $n$-manifold with boundary dense in $X$.

\section{Definition 2.17 (Normal Circuit (from [24]))}

An admissible polyhedron $X$ is called a normal circuit if its singular set $\Sigma(X)$ does not locally separate $X$ at any point, i.e. each point of $\Sigma$ has a base of neighborhoods $U$, such that $U \backslash \Sigma$ is (necessarily path) connected.

The admissible polyhedron defined in 2.14 is a normal circuit, see [25] for details.

\section{Definition 2.18 (Null set)}

By a null set on a Lip polyhedron $X$ we understand a set $Z \subset X$ such that $Z$ meets every chamber $\Omega$ (relative to some, and hence any triagulation $T=(K, \theta)$ of $X$ ) in a set whose pre-image under $\theta$ has $n$-dimensional Lebesgue measure. Denote by $\mathcal{Z}$ the collection of all null-sets $Z \subset X$.

Our admissible polyhedron $X$ is a normal circuit, it is connected as a topological space, see [25]. 


\subsection{Admissible Riemannian polyhedron}

Consider once again the structures introduced by B. Fuglede and J. Eells, [25]:

Notation 2.6 (Riemannian polyhedron) The Lip polyhedron $X$ becomes a Riemannian polyhedron $(\mathcal{M}, g)$ when endowed with a covariant bounded measurable Riemannian tensor $g_{\Omega}$, defined on each chamber $\Omega$ of $X$, satisfying the ellipticity condition (see below).

Following [29], the admissible polyhedron $X(\mathcal{M})$ becomes an admissible Riemannian polyhedron $(\mathcal{M}, g)$ when endowed with a piece(simplex)-wise smooth Riemannian metric $g$.

\subsubsection{Metric structure}

Let $T=(K, \theta)$ be a specific (Lip) triangulation of a Lip polyhedron $X$. We shall view $|K|$ as embedded in a Euclidean space $V$ via an affine Lip homeomorphism (we refer to the Lemma 4.1 from [25], one can find the proof there):

Lemma 2.1 Let $K$ be a countable finite simplicial complex of finite dimension $n$, and $V$ a Euclidean space of dimension $2 n+1$. There exists an affine Lip homeomorphism $f$ of $|K|$ onto a closed subset of $V$.

Suppose that $X$ is admissible of dimension $n$. Choose a measurable Riemannian metric $g_{\Omega}$ on the open Euclidean interior of the chamber $\theta^{-1}(\stackrel{\circ}{\Omega})$ of $|K| \subset V$, i.e. in terms of Euclidean coordinates $x^{1}, . ., x^{n}$ of points $x=\theta^{-1}(p) \in \theta^{-1}(\stackrel{\circ}{\Omega}), g_{\Omega}$ thus assigns to almost every point $p \in \stackrel{\circ}{\Omega}$ or to $x \in \theta^{-1}(\stackrel{\circ}{\Omega})$, an $n \times n$ symmetric positive matrix

$$
g_{\Omega}(x)=\left(g_{i j}^{\Omega}(x)\right)_{i, j=1, . ., n}
$$


with measurable real entries; and there is a constant $\Lambda_{\Omega} \geq 1$ such that

$$
\frac{1}{\Lambda_{\Omega}^{2}} \sum_{i=1}^{n}\left(\xi^{i}\right)^{2} \leq g_{i j}^{\Omega}(x) \xi^{i} \xi^{j} \leq \Lambda_{\Omega}^{2} \sum_{i=1}^{n}\left(\xi^{i}\right)^{2}
$$

for a.e. $x \in \theta^{-1}(\stackrel{\circ}{\Omega})$ and every $\xi=\left(\xi^{1}, . ., \xi^{n}\right) \in \mathbb{R}^{n}$.

Notation 2.7 (Uniform ellipticity) The condition (2.8) is independent on the choice of triangulation (see [25], to this end we have to define, for any, other Lip triangulation $T^{\prime}=\left(K^{\prime}, \theta^{\prime}\right.$ of $X$, the Riemannian metric $g_{\Omega^{\prime}}$ a.e. on $\theta^{\prime-1}\left(\stackrel{\Omega^{\prime}}{\Omega}\right)$ for each chamber $\Omega^{\prime}$ of $T^{\prime}$ by covariance). Condition 2.8 is independent on the choice of Euclidean frame on $\theta^{-1}(\stackrel{\circ}{\Omega})$. The second inequality in (2.8) amounts to the components of $g_{\Omega}$ being bounded. Relative to a fixed triangulation $T$ (choose such a triangulation $T$ ) of a Riemannian polyhedron $X$,

$$
\Lambda:=\sup \left\{\Lambda_{\Omega}: \Omega \in \mathcal{W}(X, T)\right\}<\infty .
$$

This is a condition of uniform ellipticity and uniform boundedness. The smallest constant $\Lambda$ in (2.9) will be called ellipticity constant of $X=(X, T, g)$.

\section{Definition 2.19 (Admissible Riemannian Polyhedron)}

A Lip admissible polyhedron $X$ (see Definition 2.14) endowed with a $C^{\infty}$-smooth covariant measurable Riemannian metric tensor $g_{i}=g\left(\Omega_{i}\right)$ on each chamber $\Omega_{i}$ satisfying the ellipticity condition (2.8) is called an Admissible Riemannian polyhedron $\mathcal{M}=(\mathcal{M}, g)$. We also assume that

$$
\left.g_{k l}\left(\Omega_{i}\right)\right|_{x \in \gamma} \neq\left. g_{k l}\left(\Omega_{j}\right)\right|_{x \in \gamma}, \quad i \neq j
$$

for each point $\boldsymbol{x} \in \gamma$, where $\gamma$ is a common interface boundary between $\Omega_{i}$ and $\Omega_{j}$.

\section{Definition 2.20 (Polyhedral Metric)}

The above covariantly defined map

$$
g: \Omega \mapsto g_{\Omega}
$$

on the set $\mathcal{W}(X, T)$ of all chambers $\Omega$ of $T$, is called the Riemannian (polyhedral) structure, or metric, on $X$. 
Remark 2.2.1 Not every admissible Riemannian polyhedron $(\mathcal{M}, g)$ can be isometrically embedded in a Euclidean space. G. De Cecco and G. Palmieri in 1993 (see [22]) constructed triangulable Riemannian Lipschitz manifold (which is an admissible Riemannian polyhedron) that does not admit any differentiale structure.

Remark 2.2.2 (Kervaire's example) The problem is that we can not consider the whole polyhedron $\mathcal{M}$ as a Riemannian manifold because of the $M$. Kervaire's results of 1960 (see his paper [40]), An example of a triangulable closed manifold of dimension 10 was constructed. That manifold does not admit any differentiable structure, actually, it does not have the homotopy type of any differentiable manifold.

Every connected open subset $U$ of a Riemannian polyhedron $\mathcal{M}$ can be considered as an interior of a Riemannian polyhedron with piece-wise smooth boundary and the induced Riemannian structure.

Notation 2.8 We consider closed, compact admissible Riemannian polyhedron $\mathcal{M}$, with piece-wise $C^{\infty}$-smooth metric $g$ on it, its boundary $\partial \mathcal{M}=\bigcup \Gamma_{i}, i=1, \ldots, N_{1}$, where $\Gamma_{i}$ are $(n-1)$-simplices that belong just to one chamber $\Omega_{m}, m=1, \ldots, M$. We denote interface boundaries by $\gamma_{i}$, or $\gamma$. Denote the set of all interface boundaries of the manifold $\mathcal{M}$ by

$$
I B(\mathcal{M}):=\bigcup_{j=1}^{N_{2}} \gamma_{j}=W P^{n-1} \backslash \partial \mathcal{M}, \quad N_{2}<\infty .
$$

We call the union of all $k$-simplices $W P^{k}, k=0, \ldots n-2$, as well we can call them wedge points of $\mathcal{M}$. We call chambers $(n$-simplices of $\mathcal{M}) \Omega_{j}, j=1, M$, the interior of chambers will be denoted by $\stackrel{\circ}{\Omega}$, or, equivalently, $\Omega^{i n t}$. We call the $W P^{0}$ the union of all conical points of $\mathcal{M}$, as before, $\mathcal{W}=\bigcup_{i} \Omega_{i}$.

Notation 2.9 (Chambers) We need some special notations for chambers. In the case when we need just two of them (when we consider, for instance, the vicinity of any interface $\gamma$ ) we would name them $\Omega_{-}$and $\Omega_{+}$. The corresponding metrics are $g^{ \pm}=g\left(\Omega_{ \pm}\right)$, see Figure 2.3. 


\begin{tabular}{|l|l|l|}
$\Omega_{1}, g^{1}$ & $\Omega_{2}, g^{2}$ & $\Omega_{3}, g^{3}$ \\
\hline$\Omega_{6}, 9^{6}$ & $\Omega_{5}, 9^{5}$ & $\Omega_{4}, g^{4}$ \\
\hline
\end{tabular}

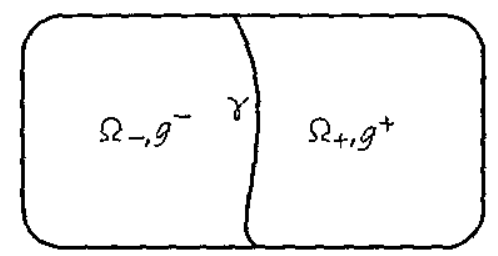

Figure 2.3: Various notations of chambers

In the present work we assume that all interfaces of the admissible Riemannian polyhedron satisfy to $(2.10)$.

\section{Definition 2.21 (Artificial interfaces)}

When condition 2.10 is not valid not for all points on the interface $\gamma$, this is not the case we consider in the present thesis. We call the interface artificial interface for the smoothly glued metric tensors.

There exists another important case is the case when metric tensors of adjacent chambers are glued continuously on the interface. This is the case of gluing by isometry when we assume that metric is continuous but its normal derivatives have jumps. Considerations of that kind of interfaces are beyond the scope of the present work. 


\subsubsection{Coordinates}

We need to introduce several types of local coordinates on $\mathcal{M}$, let us start with

Notation 2.10 (Inner coordinates) Any regular (inner) local coordinates

$$
\left(x^{1}, \ldots, x^{n}\right)=\left\{x^{i}\right\}=\mathbf{x}, i=1, \ldots, n
$$

which are smooth inside some chamber $\Omega^{\text {int }}$ (in some chart on $\mathcal{M}$ ). From now on we will mostly use Latin indices to count coordinates with respect to full dimension $n$.

Notation 2.11 (Semi-geodesic Coordinates) The second type of coordinates are boundary normal, or semi-geodesic coordinates, (see [6], [7], [37]), i.e. corresponding to the interface boundaries $\gamma$ (see the rigth hand side of Figure 2.3), such that $(\mathbf{q}, \sigma)=$ $\left(\mathbf{q}, \sigma^{ \pm}\right)=\left(q^{1}, \ldots, q^{n-1}, \sigma\right)=\left\{q^{\alpha}, \sigma\right\}_{\alpha=1}^{n-1}$, where $\mathbf{q}$ are some smooth coordinates on the interface $\gamma$, and $\sigma^{ \pm}$is the distance to $\gamma$ with respect to the metric $g^{ \pm}$, i.e.

$$
\sigma= \begin{cases}\sigma^{+}(x)>0, & x \in \Omega_{+}, \\ \sigma(x)=0, & x \in \gamma, \\ -\sigma^{-}(x)<0, & x \in \Omega_{-} .\end{cases}
$$

We will use Greek letters $\alpha, \beta, \delta, \ldots$ to count $(n-1)$ interface coordinates $\left\{q^{\alpha}\right\}=$ $\left\{q^{1}, \ldots, q^{n-1}\right\}$. We choose the origin $M_{1}$ of semi-geodesic coordinates to belong to $\gamma$, so we choose point $M_{1}$ to have coordinates $\sigma=0, q^{\alpha}=0$.

We can choose these coordinates in the vicinity of $\gamma$ by the following procedure. Choose local coordinate system (map) $\left(q^{1}, \ldots, q^{n-1}\right)$ on $\gamma$ containing point $M_{1}$. We introduce local coordinates $\left(x^{1}, \ldots, x^{n}\right)$ on $\Omega_{-}$such that $x^{n}(P)=\sigma_{-}(P)$ is the distance from the point $P \in \Omega_{-}$to $\gamma$ in metric $g^{-}$, corresponding to $\Omega_{-}$and the rest of point $P$ coordinates are the same as the coordinates of the closest to $P$ point on $\gamma$. Similarly, the distance in $\Omega_{+}$from $P$ to $\gamma$ is equal to $-\sigma_{-}(P)$, and the rest coordinates are the same as before. Together these coordinates give coordinates smooth on $\Omega_{-}$and $\Omega_{+}$ 
separately in a small vicinity of $\gamma$ on $X$, consequently they give smooth structure on $X$ where $\Omega_{-}$and $\Omega_{+}$appear to be smooth sub-manifolds.

As $\sigma$ is orthogonal to all $q^{\alpha}$ the matrix of metric tensor has the following form in semi-geodesic coordinates:

$$
\left\{g_{i j}^{ \pm}\right\}=\left(\begin{array}{cc}
g_{\alpha \beta}^{ \pm} & 0 \\
0 & 1
\end{array}\right)
$$

where

$$
g_{\alpha \beta}^{ \pm}=\left(\frac{d \mathbf{x}}{d q^{\alpha}}, \frac{d \mathbf{x}}{d q^{\beta}}\right)_{g^{ \pm}}
$$

is $(n-1) \times(n-1)$-smooth matrix of tangent components of metric.

Notation 2.12 (Boundary normal coordinates) We will use various notations for the semi-geodesic coordinates depending on the basic hyper-surfaces. In the case when the their tangential part lives on some interface $\gamma$ we will denote them as above, $\mathrm{q} \in \gamma$ and $\sigma$ the normal coordinate. In the case when their tangential part lives in the part of the polyhedron boundary $\Gamma \subset \partial \mathcal{M}$, or in the boundary of some smooth subset $\mathcal{D}$ of $\mathcal{M}$, we will denote them by $z \in \Gamma\left(\left\{z^{\alpha}\right\}_{\alpha=1}^{n-1}=\left(z^{1}, \ldots, z^{n-1}\right) \in \Gamma\right)$, and $\tau$ (or $s$ ) the normal coordinate, pointing inside the manifold. In the latter case we call these coordinates the boundary normal coordinates. 


\subsection{Distance structure on $(\mathcal{M}, g)$}

This subchapter contains a description of methods of introducing a distance structure on an admissible Riemannian polyhedra from [25]. Then, adapting the construction of G. De Cecco and G. Palmieri (see [19]-[22]) an intrinsic distance $d_{X}$ (Caratheodori distance) is defined on $X$, which thereby becomes a length space, and hence a geodesic space (see Statement 2.1.0.1).

Remark 2.3.1 We present most of the results in assumption that our metric tensor is non-smooth being only measurable. All proofs presented are for the case of locally finite polyhedra as the results can be used for the case of Admissible Riemannian polyhedron as well.

\subsubsection{Intrinsic distance $d_{X}$}

Notation 2.13 (Euclidean Riemannian metric $g^{e}$ ) In addition to the piece-wise smooth metric tensor $g$ on $\mathcal{M}$, we shall always consider the Euclidean Riemannian metric $g^{e}$ on a Lip polyhedron $X$ (corresponding to $\mathcal{M}$ ) with a specified triangulation $T=(K, \theta)$. For each $\Omega \in \mathcal{W}(X, T), g_{\Omega}^{e}$ is defined in terms of a Euclidean frame on $\theta^{-1}\left(\Omega^{i n t}\right)$ by the unit matrix $\delta_{i j}$. Thus $g^{e}$ is by no means covariantly defined and should be regarded as a mere reference metric on $(X, T)$.

Remark 2.3.2 The ellipticity constant $\Lambda$ of $\mathcal{M}=(X, T, g)$ from (2.9) equals to the bi-Lip constant in the identity map $(X, T, g) \rightarrow\left(X, T, g^{e}\right)$ in terms of the associated intrinsic distance $d_{\mathcal{M}}=d_{\mathcal{M}}^{9}$ and its analogue $d_{X}^{e}$, both of which are determined below.

Relative to a given (Lip) triangulation $T=(K, \theta)$ of an $n$-dimensional admissible Riemannian polyhedron $(\mathcal{M}, g)$, we have on $\mathcal{M}$ the distance function $e$ induced by the Euclidean distance on a Euclidean space $V$ in which $|K|$ is affinely Lip embedded, 
cf. Lemma 2.1. This distance $e$ on $\mathcal{M}$ is not intrinsic and will play an auxiliary role in defining an equivalent intrinsic distance $d_{\mathcal{M}}^{g}=d_{\mathcal{M}}$ as follows, by a slight adaptation of the procedure used by G. De Cecco and G. Palmieri [19] for the case of Riemannian Lipschitz manifold.

Consider $\mathcal{Z}$ defined above, see Definition 2.18. For a given triangulation $T=(K, \theta)$ consider in particular the set $Z_{T} \in \mathcal{Z}$ obtained from $\mathcal{M}$ by removing from each chamber $\Omega$ in $\mathcal{M}$ those points of $\stackrel{o}{\Omega}$ which are Lebesgue points of $g^{\Omega}$ (that is, for every component $g_{i j}^{\Omega}$ measurable, see Remark 2.3.1).

Notation 2.14 (Family of paths $\operatorname{Lip}^{Z}(x, y ; \mathcal{M})$ ) We denote by $\operatorname{Lip}^{Z}(x, y ; \mathcal{M})$ the family of all Lip continuous paths $\eta:[a, b] \rightarrow(\mathcal{M}, e)$ with $\eta(a)=x, \eta(b)=y$ which are transversal to $Z$ in the sense that $\eta^{-1}(Z)$ is a null subset of $[a, b]$ for any two points $x, y \in \mathcal{M}$ and any $Z \in \mathcal{Z}$ such that $Z \supset Z_{T}$.

The length $L_{T}(\eta)$ of such a path $\eta$ is well defined by

$$
L_{T}(\eta)=\sum_{\Omega \in \mathcal{W}(\mathcal{M}, T)} \int_{\eta^{-1}\left(\Omega^{i n t}\right)} \sqrt{\left(g_{i j}^{\Omega} \circ \theta^{-1} \circ \eta\right) \dot{\eta}^{i} \dot{\eta}^{j}} \leq \infty
$$

where $\left(\eta^{1}, \ldots, \eta^{n}\right)=\theta^{-1} \circ \eta$ in terms of Euclidean coordinates on the open Euclidean simplex $\theta^{-1}\left(\Omega^{i n t}\right)$, and the dot means differentiation.

Write

$$
\rho_{Z}(x, y)=\inf _{\eta}\left\{L_{T}(\eta): \eta \in \operatorname{Lip}^{Z}(x, y ; \mathcal{M})\right\}
$$

Here $\rho_{Z}$ depends also on $T$. Clearly, $Z_{1} \supset Z_{2}\left(\supset Z_{T}\right)$ implies $\rho_{Z_{1}}(x, y) \geq \rho_{Z_{2}}(x, y)$. Finally set

$$
d_{\mathcal{M}}(x, y)=\sup _{Z}\left\{\rho_{Z}(x, y): Z \in \mathcal{Z}, Z \supset Z_{T}\right\}
$$

The Euclidean segment $[x, y] \subset \Omega$ can be slightly deformed to a path

$$
\eta=[x, z] \bigcup[z, y] \subset \Omega
$$


such that $\eta^{-1}(Z)$ is null. Clearly, $\rho_{Z}$ satisfies the triangle inequality, and so therefore does $d_{\mathcal{M}}$.

For the Euclidean Riemannian metric $g^{e}$ on $\mathcal{M}$ (induced by the Euclidean distance $e$ on $V$ ) we have similar notions $L_{T}^{e}(\eta), \rho_{Z}^{e}(x, y), d_{\mathcal{M}}^{e}(x, y)$. In view of (2.9), each of quotients

$$
\frac{L_{T}(\eta)}{L_{T}^{e}(\eta)}, \frac{\rho_{Z}(x, y)}{\rho_{Z}^{e}(x, y)}, \frac{d_{\mathcal{M}}(x, y)}{d_{\mathcal{M}}^{e}(x, y)}
$$

lie between $\Lambda^{-1}$ and $\Lambda$. Then

$$
e(x, y) \leq d_{X}^{e}(x, y), \text { for } x, y \in X
$$

Here the sign of equality holds if $x$ and $y$ are in the same simplex $s$. This can be shown by deforming $[x, y]$ into $\eta=[x, z] \cup[z, y]$ with $\eta^{-1}(Z)$ null and

$$
L_{T}^{e}(\eta) \leq e(x, y)+\varepsilon
$$

whereby $\rho_{Z}(x, y)$ and hence $d_{\mathcal{M}}^{e}(x, y)$ are $\leq e(x, y)+\varepsilon$.

Since $\mathcal{M}(X)$ is connected it follows that $d_{X}^{e}(x, y)<\infty$ and hence $d_{\mathcal{M}}(x, y)<\infty$ for any pair $x, y \in \mathcal{M}$. By $(2.14), d_{X}^{e}(x, y)>0$ and hence $d_{\mathcal{M}}(x, y)>0$ when $x \neq y$. Altogether, $d_{\mathcal{M}}$ and $d_{X}^{e}$ are equivalent metrics on $\mathcal{M}$, depending on a priori triangulation $T$. They are locally equivalent to the given metric on $\mathcal{M}$ as a Lip polyhedron according to Lemma 2.2 below, applied to the star of any point of $\mathcal{M}$.

Remark 2.3.3 (Intrinsic distance) In view of Lemma 2.3 below, $d_{\mathcal{M}}$ is called the intrinsic distance on $(\mathcal{M}, g)$.

\section{Proposition 2.3.1}

Distance $d_{X}^{e}$ is continuous and hence bounded as a function on $X \times X$ due to compactness, see [25].

Proof of Proposition 2.3.1. Indeed, for given $x_{0}, y_{0} \in X$ and for any $x \in \operatorname{st}\left(x_{0}\right), y \in$ st $\left(y_{0}\right)$, we have $d_{X}^{e}\left(x, x_{0}\right)=e\left(x, x_{0}\right), d_{X}^{e}\left(y, y_{0}\right)=e\left(y, y_{0}\right)$, as noted after (2.14); and 
so

$$
\left|d_{X}^{e}(x, y)-d_{X}^{e}\left(x_{0}, y_{0}\right)\right| \leq e\left(x, x_{0}\right)+e\left(y, y_{0}\right) \rightarrow 0
$$

as $(x, y) \rightarrow\left(x_{0}, y_{0}\right)$ in $X \times X$

Lemma 2.2 Let $X$ be a finite Lip polyhedron, affinely and Lip homeomorphically embedded in a Euclidean space $V$. The induced Euclidean distance $e$ on $X$ is then equivalent to the distance $d_{X}^{e}$ associated with the Euclidean Riemannian structure $g^{e}$ on $X$, hence also equivalent to the intrinsic distance $d_{\mathcal{M}}$ associated with a given Riemannian metric $g$ on $\mathcal{M}$.

Proof of Lemma 2.2. We have $d_{\mathcal{M}}$ and $d_{X}^{e}$ are equivalent distances, see also Proposition 2.3.1. In view of (2.14) it remains to show that $d_{X}^{e} / e$ remains bounded on $X \times X$. Suppose there are sequences $\left(x_{j}\right),\left(y_{j}\right) \subset X$ such that

$$
\frac{d_{X}^{e}\left(x_{j}, y_{j}\right)}{e\left(x_{j}, y_{j}\right)} \rightarrow \infty \text { as } j \rightarrow \infty
$$

Since $d_{X}^{e}$ is bounded on $X \times X, e\left(x_{j}, y_{j}\right) \rightarrow 0$ as $j \rightarrow \infty$. Passing subsequences may assume that there exists $a \in X$ such that $x_{j} \rightarrow a, y_{j} \rightarrow a$. Let $\omega_{0}$ denote the carrier of $a$, i.e., the lowest dimensional simplex of $(X, T)$ containing $a$ (necessary as an inner point). Let $s$, resp. $t$, denote the lowest dimensional simplex, containing $\omega_{0}$ and also infinitely many $x_{j}$, resp. $y_{j}$; we may assume that $x_{j} \in s, y_{j} \in t$ for all $j$. Using $a$ as an origin, consider in the Euclidean space $V$ the linear subspaces $V_{\omega_{0}}, V_{s}, V_{t}$ spanned by $\omega_{0}, s, t$ respectively. Denoting by $x^{\prime}$ the orthogonal projection of the point $x \in V$ on $V_{\omega_{0}}$, we may further assume that $x_{j}^{\prime}, y_{j}^{\prime} \in \omega_{0}$. If $s \neq t$ we finally arrange that $V_{s}$ and $V_{t}$ are perpendicular to one another in the sense that for every $x \in V_{s}, y \in V_{t}$, the vectors $x-x^{\prime}$ and $y-y^{\prime}$ are orthogonal; for if this is not already the case, it can be achieved by applying a linear, hence bi-Lip, bijection of $V$ onto itself. In terms of the Euclidean norm $|\cdot|$ on $V$ we then have

$$
d_{X}^{e}\left(x_{j}, y_{j}\right) \leq\left|x_{j}-x_{j}^{\prime}\right|+\left|x_{j}^{\prime}-y_{j}^{\prime}\right|+\left|y_{j}^{\prime}-y_{j}\right| \leq 3\left|x_{j}-y_{j}\right|=3 e\left(x_{j}, y_{j}\right)
$$


nothing that the vectors $x_{j}-x_{j}^{\prime} \in V_{s} \ominus V_{\omega_{0}}, x_{j}^{\prime}-y_{j}^{\prime} \in V_{\omega_{0}}$ and $y_{j}^{\prime}-y_{j} \in V_{t} \ominus V_{\omega_{0}}$, are mutually orthogonal with the sum $x_{j}-y_{j}$. The contradiction with (2.16) completes the proof, the remaining case $s=t$ being trivial, again see text following (2.14).

Notation 2.15 $(\operatorname{Lip}(\mathcal{M}))$ The class $\operatorname{Lip}(\mathcal{M})$ is the class of Lip continuous functions $u: \mathcal{M} \rightarrow \mathbb{R}$ (using any of local distances on $\mathcal{M}$ considered above). The gradient $|\nabla u|$ is the Riemannian gradient, defined a.e. in $\mathcal{M}$ (that is a.e. in each $\Omega \in \mathcal{W}(\mathcal{M}, T)$ ), for a given triangulation $(K, \theta)$ of $\mathcal{M}$, by Rademacher's theorem for Lip functions on Euclidean domains, applied to $u$ expressed in Euclidean coordinates in the interior of $\theta^{-1}(\Omega)$.

Notation 2.16 $(\operatorname{Lip}(x, y ; \mathcal{M}))$ Also $\operatorname{Lip}(x, y ; \mathcal{M})$ denotes the family of all Lip paths $\eta:[a, b] \rightarrow\left(\mathcal{M}, d_{\mathcal{M}}\right)$ joining $x$ to $y$.

Lemma 2.3 (Distance function on $\mathcal{M}$ ) 1. The distance function $d_{\mathcal{M}}=d_{\mathcal{M}}^{g}$ on an admissible Riemannian polyhedron $(\mathcal{M}, g)$ is intrinsic, in particular independent of the chosen triangulation.

2. $(\mathcal{M}, g)$ is a length space (hence a geodesic space, if complete).

3. $d_{\mathcal{M}}$ equals Caratheodory distance

$$
d_{\mathcal{M}}(x, y)=\max \{|u(x)-u(y)|: u \in \operatorname{Lip}(\mathcal{M}),|\nabla u| \leq 1 \text { a.e. in } \mathcal{M}\}
$$

Proof of Lemma 2.3 (see [25]). First note that for given $x, y \in \mathcal{M}$, there exists a null set $Z_{T}^{*} \in \mathcal{Z}$ with $Z_{T}^{*} \supset Z_{T}$ and large enough so that

$$
\rho_{Z_{T}^{*}}(x, y)=d_{\mathcal{M}}(x, y)
$$

(It suffices to choose an increasing sequence $\left(Z_{j}\right) \subset \mathcal{Z}$ with $Z_{j} \supset Z_{T}$ so that $\rho_{Z_{j}}(x, y) \rightarrow d_{\mathcal{M}}(x, y)$, and to take $Z_{T}^{*}=\bigcup_{j} Z_{j}$, noting that $Z_{T}^{*} \supset Z_{T}$ and $\rho_{Z_{T}^{*}}(x, y) \geq$ $\rho_{Z_{j}}(x, y)$.) 
(1) Fix $x, y \in \mathcal{M}$, and consider any triangulation $T^{\prime}=\left(K, \theta^{\prime}\right)$. For any chamber $\Omega_{\alpha} \in \mathcal{W}(\mathcal{M}, T)$ and any chamber $\Omega_{\beta}^{\prime} \in \mathcal{W}\left(\mathcal{M}, T^{\prime}\right)$ such that $U:=\Omega_{\alpha} \cap \stackrel{\circ}{\Omega_{\beta}^{\prime}} \neq \emptyset$ we have the Lip homeomorphism

$$
\left.\theta^{\prime-1} \circ \theta\right|_{\theta^{-1}(U)}: \theta^{-1}(U) \rightarrow \theta^{-1}(U)
$$

between the open subsets $\theta^{-1}(U)$ and $\theta^{\prime-1}(U)$ of the Euclidean simplexes $\theta^{-1}\left(\Omega_{\alpha}^{\text {int }}\right)$ and $\theta^{\prime-1}\left(\Omega_{\beta}^{\prime \text { int }}\right)$. This Lip homeomorphism is differentiable off some Lebesgue null set $Z_{\alpha \beta} \subset \theta^{-1}(U)$, mapped by $\theta$ onto a null set $\theta\left(Z_{\alpha \beta}\right) \subset U$. Writing

$$
Z_{0}=Z_{T} \cup Z_{T^{\prime}} \cup \bigcup_{\alpha, \beta} \theta\left(Z_{\alpha \beta}\right)(\in \mathcal{Z})
$$

we have for any $Z \in \mathcal{Z}$ with $Z \supset Z_{0}$ and any path $\eta \in \operatorname{Lip}^{Z_{0}}(x, y ; \mathcal{M})$

$$
L_{T}(\eta)=L_{T^{\prime}}(\eta)
$$

because this holds by covariance for the contributions from $\eta^{-1}\left(\Omega_{\alpha} \cap \Omega_{\beta}^{\prime}\right)$ to $L_{T}(\eta)$ and to $L_{T^{\prime}}(\eta)$.

For any $Z \in \mathcal{Z}$ with $Z \supset Z_{T^{\prime}}$ we have the quantities $\rho_{Z}^{\prime}(x, y)$ and $d_{\mathcal{M}}^{\prime}(x, y)$ corresponding to $\rho_{Z}(x, y)$ and $d_{\mathcal{M}}(x, y)$, respectively, but relative to $T^{\prime}$ in place of $T$. For any path $\eta \in \operatorname{Lip}^{Z \cup Z_{0}}(x, y ; \mathcal{M})$ we obtain

$$
\rho_{Z}^{\prime}(x, y) \leq \rho_{Z \cup Z_{0}}^{\prime}(x, y) \leq L_{T^{\prime}}(\eta)=L_{T}(\eta) .
$$

By varying $\eta \in \operatorname{Lip}^{Z \cup Z_{0}}(x, y ; \mathcal{M})\left(\subset \operatorname{Lip}^{Z_{0}}(x, y ; \mathcal{M})\right)$ we conclude that

$$
\rho_{Z}^{\prime}(x, y) \leq \rho_{Z \cup Z_{0}}(x, y) \leq d_{\mathcal{M}}(x, y)
$$

and finally $d_{\mathcal{M}}^{\prime}(x, y) \leq d_{\mathcal{M}}(x, y)$, similarly $d_{\mathcal{M}}(x, y) \leq d_{\mathcal{M}}^{\prime}(x, y)$.

(2) With the intrinsic distance we associate in the standard way the intrinsic length $L(\eta)$ of a Lip path $\eta:[a, b] \rightarrow\left(\mathcal{M}, d_{\mathcal{M}}\right)$ (or equivalently $\eta:[a, b] \rightarrow(X, e)$, by Lemma 2.2 , which is applicable because only finitely many chambers of $\mathcal{M}$ meet the compact image $\eta([a, b]))$ :

$$
L(\eta)=\sup _{\pi} \sum_{i=1}^{k} d_{\mathcal{M}}\left(x_{i-1}, x_{i}\right),
$$


where $\pi$ ranges over all subdivisions of $[a, b]$ :

$$
a=x_{0}<x_{1}<\ldots<x_{k-1}<x_{k}=b .
$$

Returning to the null set $Z_{T}^{*}$ from (2.17) (for given $x, y \in \mathcal{M}$ and a given triangulation $T$ of $\mathcal{M}$ ) we may clearly choose $Z=Z_{T}^{*} \in \mathcal{Z}$ so as to satisfy (2.17) for all pairs of points $x, y$ from a countable dense subset $\mathcal{M}^{*}$ of $\mathcal{M}$. With that set $Z$ we show that

$$
L(\eta) \leq L_{T}(\eta)
$$

for any Lip path $\eta:[a, b] \rightarrow(\mathcal{M}, e)$ such that $\eta^{-1}(Z)$ is null. Via a subdivision of $[a, b]$ this in fact reduces to $d_{\mathcal{M}}(x, y) \leq L_{T}(\eta)$. For given $\varepsilon>0$ choose $x^{*}, y^{*} \in$ $\mathcal{M}^{*}$ so that $d_{\mathcal{M}}\left(x, x^{*}\right), d_{\mathcal{M}}\left(y, y^{*}\right)<\varepsilon$. By the definition of $\rho_{Z}(x, y)$, it is less than $\left.d_{\mathcal{M}}(x, y)\right)$, and there are paths $\alpha \in \operatorname{Lip}^{Z}\left(x^{*}, x ; \mathcal{M}\right)$ and $\beta \in \operatorname{Lip}^{Z}\left(y^{*}, y ; \mathcal{M}\right)$ such that $L_{T}(\alpha), L_{T}(\beta)<\varepsilon$. The path $\eta^{*}=\alpha \cup \eta \cup \beta$ belongs to $\operatorname{Lip}^{Z}\left(x^{*}, y^{*} ; \mathcal{M}\right)$, and by the choice of $Z$ above,

$$
d_{\mathcal{M}}(x, y) \leq d_{\mathcal{M}}\left(x^{*}, y^{*}\right)+2 \varepsilon=\rho_{Z}\left(x^{*}, y^{*}\right)+2 \varepsilon \leq L_{T}\left(\eta^{*}\right)+2 \varepsilon \leq L_{T}(\eta)+4 \varepsilon .
$$

This shows that $d_{\mathcal{M}}(x, y) \leq L_{T}(\eta)$, thus establishing (2.19).

We are now prepared to show that $\left(\mathcal{M}, d_{\mathcal{M}}\right)$ is a length space because

$$
d_{\mathcal{M}}(x, y)=\inf \{L(\eta): \eta \in \operatorname{Lip}(x, y ; \mathcal{M})\}
$$

for every $x, y \in \mathcal{M}$. Choosing $Z=Z_{T}^{*} \in \mathcal{Z}$ conforming with $(2.17)$ we then have from (2.19) when $\eta$ ranges over $\operatorname{Lip}^{Z}(x, y ; \mathcal{M})$

$$
\inf _{\eta} L(\eta) \leq \inf _{\eta} L_{T}(\eta)=\rho_{Z}(x, y)=d_{\mathcal{M}}(x, y)
$$

A fortiori, the inequality sign $\geq$ holds in (2.20). The opposite inequality in $(2.20)$ is obvious.

(3) Let $\delta(x, y)$ denote the supremum of $|u(x)-u(y)|$ for the stated functions $u$. For any such function $u$ take for $Z$ the union of $Z_{T}$ and the null set of points $x$ of $\mathcal{M} \backslash Z_{T}$ 
at which $u$ is not differentiable (as a function on $\Omega^{\text {int }} \ni x, \Omega \in \mathcal{W}(\mathcal{M}, T)$ ) with $|\nabla u| \leq 1$. For any $\eta \in \operatorname{Lip}_{Z}(x, y ; \mathcal{M})$ we then have

$|u(x)-u(y)|=\left|\sum_{\Omega \in \mathcal{W}(\mathcal{M}, T)_{\eta^{-1}\left(\Omega^{i n t}\right)}} \frac{d}{d t}(u \circ \eta)\right| \leq \sum_{\Omega \in \mathcal{W}(\mathcal{M}, T)_{\eta^{-1}\left(\Omega^{i n t}\right)}}|\langle(\nabla u) \circ \eta, \dot{\eta}\rangle| \leq L_{T}^{Z}(\eta)$ by Cauchy-Schwarz, $\langle\cdot, \cdot\rangle$ and $|\cdot|$ denoting the Riemannian metric and the norm on (the tangent bundle of) $\Omega^{\text {int }}$ for each $\Omega \in \mathcal{W}(\mathcal{M}, T)$. It follows that $|u(x)-u(y)| \leq$ $\rho_{Z}(x, y) \leq d_{\mathcal{M}}(x, y)$.

In the opposite direction, note that the Lip function $u: d_{\mathcal{M}}(x, \cdot)$ on $\mathcal{M}$ completes in the definition of $\delta(x, y)$ because $|\nabla u| \leq 1$ a.e. in each $\Omega^{i n t}, \Omega \in \mathcal{W}(\mathcal{M}, T)$, by the triangle inequality, noting also that $d_{\mathcal{M}}(x, y)$ equals the usual Riemannian distance between two points $x, y \in \Omega^{i n t}$. (This is because the usual geodesic segment $[x, y]$ can be slightly modified to a path, e.g., $[x, z] \cup[z, y]$ of class $\operatorname{Lip}^{Z}(x, y ; \mathcal{M})$ and the length only slightly bigger than that of $[x, y]$, by the usual Fubini argument, assuming $y$ near $x$.) This ends the proof.

Also the following Lemma has been proved.

Lemma 2.4 Thus for any triangulable admissible Riemannian polyhedron $(\mathcal{M}, g)$ the intrinsic distance $d_{\mathcal{M}}(x, y)$ introduced above in (2.20) equals the usual Riemannian (Lip) distance between $x$ and $y$. Also, $(\mathcal{M}, g)$ is a geodesic space due to the Statement 2.1.0.1, thus any two points on $\mathcal{M}$ can be joined by a Lip continuous rectifiable path $\eta$, such that $L(\eta)$ (2.18) is a distance between these points, and $\eta$ has a Lesbegue measure zero intersection with all null sets (2.18) of $X$. 


\subsection{Laplace-Beltrami operator}

On an admissible Riemannian polyhedron $\mathcal{M}$ the Sobolev space $W_{1}^{2}(\mathcal{M})$, defined as a completion of a suitable space of Lipschitz continuous functions in the Sobolev norm, is a Dirichlet space. We remember that $d_{\mathcal{M}}$ is an intrinsic distance on $(\mathcal{M}, g)$.

Notation $2.17\left(\operatorname{Lip}^{1,2}(\mathcal{M})\right.$ ) We denote by $\operatorname{Lip}^{1,2}(\mathcal{M})$ the linear space of all Lip continuous functions $u:\left(\mathcal{M}, d_{\mathcal{M}}\right) \rightarrow \mathbb{R}$ for which the Sobolev $([50])(1,2)-$ norm $\|u\|$ defined by

$$
\|u\|^{2}=\int_{\mathcal{M}}\left(u^{2}+|\nabla u|^{2}\right)
$$

is finite for $\mathcal{M}$ compact, here $\nabla u$ the Riemannian length of the Riemannian gradient on each $\Omega_{s}$. Here the integration is taken with respect to the Riemannian volume measure on $\mathcal{M}$, i.e. on each $\Omega_{s} \in W P^{n}(\mathcal{M})$. By Randemacher's theorem $\nabla u$ exists a.e. in each $\Omega_{s}$ (hence a.e in $\mathcal{M}$ ), see [28], [27], [54], [55], [61], [52].

As metrics $g_{i}$ on each chamber $\Omega_{i}$ are smooth up to the boundary, we introduce a map which is $H^{1}(\Omega)$ smooth, then we consider our polyhedron $\mathcal{M}$ as a collection of chambers and obtain functional class $H^{1}(\mathcal{M})$ as a closure of all Lipschitz functions on $\mathcal{M}$. We introduce a Dirichlet integral, i.e. a quadratic form in $L_{2}(\mathcal{M})$ by the following

$$
Q^{D}[u]:=\sum_{\Omega_{s} \in W P^{n}(\mathcal{M})} \int_{\Omega_{s}}\left(u^{2}+|\nabla u|^{2}\right),\left.u\right|_{\partial \Omega_{s}}=\left.u\right|_{\partial \Omega_{s^{\prime}}}
$$

where we considered $u$ as a finite set of $H^{1}$-smooth functions on each chamber, $W P^{n}(\mathcal{M})$ denoting the collection of all chambers $\Omega_{s}$ of $\mathcal{M}$ (relative to a given triangulation). The Dirichlet integral $Q^{D}$ introduced by (2.22) is positively definite in $L_{2}(\mathcal{M})$, semi-bounded from below, then there exists a self-adjoint Euler-Lagrange operator corresponding to it, which is locally a Laplace-Beltrami operator,

$$
\Delta u=\Delta_{g} u=g^{-\frac{1}{2}} \partial_{i}\left(g^{i j}\left(g^{\frac{1}{2}} \partial_{j} u\right)\right.
$$


where $\partial_{i}=\frac{\partial u}{\partial x^{i}}, i=1, \ldots, n$, and $g:=\operatorname{det}\left(g_{i j}\right)$, and $\left(g^{i j}\right)=\left(g_{i j}\right)^{-1}$. Consider now admissible Riemannian polyhedron $(\mathcal{M}, g)$, where metric $g$ is determined above. In general, there is no selected coordinate system on $\mathcal{M}$ but one can still define the Laplace operator locally in any chart x inside each $\Omega_{m}$ by using (2.23), where $g_{i j}^{m}$ is now the Riemannian metric tensor on $\Omega_{m}$ (which as we saw above determines the length by (2.18)) and locally on $\gamma$ for $\sigma=0$. Definition (2.23) is covariant, that is, in any other chart this operator will have the same form. We should consider what's happening on the interfaces. Now we consider the Laplace-Beltrami operator $\Delta_{ \pm}$ locally on each side of $\gamma$ (see Notation 2.9), which in local semi-geodesic coordinates has the form

$$
\Delta_{g}=\Delta_{ \pm}=\frac{1}{\sqrt{g}}\left(\partial_{\alpha} g^{\alpha \beta} \sqrt{g} \partial_{\beta}+\partial_{n} \sqrt{g} \partial_{n}\right), \quad g=\operatorname{det} g_{i j}, i, j=1, . ., n
$$

where $\partial_{\alpha}:=\frac{\partial}{\partial q^{\alpha}}, \partial_{n}:=\partial_{\sigma}:=\frac{\partial}{\partial \sigma}$ and

$$
g=\left\{\begin{array}{l}
g^{+}, \sigma>0 \\
g^{-}, \sigma<0
\end{array} \quad, \text { or } g=\left\{\begin{array}{l}
g^{+},\left(\mathbf{q}, \sigma^{+}\right) \in \Omega_{+} \\
g^{-},\left(\mathbf{q}, \sigma^{-}\right) \in \Omega_{-} .
\end{array}\right.\right.
$$

We define $H_{0}^{1}(\mathcal{M})$ to be the closure of $C_{0}^{\infty}(\mathcal{M})$ in $W_{1}^{2}(\mathcal{M})$.

\section{Definition 2.22 (Weak solution to the Laplace-Beltrami equation)}

We define a weak solution to the following Problem

$$
\Delta u=f,\left.\quad u\right|_{\partial \mathcal{M}}=0,
$$

as a function $u \in H_{0}^{1}(\mathcal{M})$ that satisfies the equation $\Delta u=f$ in the sense of distributions. This is equivalent to the integral inequality

$$
-\int_{\Omega} \nabla u \cdot \nabla v=\int f v, \forall v \in H_{0}^{1}(\mathcal{M}),
$$

$u$ is then from the domain of Dirichlet Laplace-Beltrami operator.

Let us determine the domain of the Dirichlet Laplace-Beltrami operator for the weak solutions on admissible Riemannian polyhedra. Consider for simplicity the integration 
over $\mathcal{M}=\Omega_{-} \cup \Omega_{+}$, as there are finite number of interfaces we can generalize the result for all of them afterwards.

$$
I_{1}:=\int_{\Omega}(\Delta u) v \sqrt{g} d x=\int_{\Omega} \frac{1}{\sqrt{g}} \partial_{i}\left(g^{i j} \sqrt{g} \partial_{j} u\right) v \sqrt{g} d x .
$$

Now we substitute the form of the Laplace-Beltrami operator (2.24) near the interface and separate the integral two integrals over $\Omega_{-}$ans $\Omega_{+}$, thus

$$
\begin{aligned}
I_{1} & =\int_{\Omega_{-}}\left(\partial_{\alpha} g_{-}^{\alpha \beta} \sqrt{g^{-}} \partial_{\beta} u\right) v(q, \sigma) d q d \sigma+\int_{\Omega_{+}}\left(\partial_{\alpha} g_{+}^{\alpha \beta} \sqrt{g^{+}} \partial_{\beta} u\right) v(q, \sigma) d q d \sigma \\
& +\int_{\Omega_{-}}\left(\partial_{\sigma}\left(\sqrt{g^{-}} \partial_{\sigma} u\right)\right) v(q, \sigma) d q d \sigma+\int_{\Omega_{+}}\left(\partial_{\sigma}\left(\sqrt{g^{+}} \partial_{\sigma} u\right)\right) v(q, \sigma) d q d \sigma .
\end{aligned}
$$

The first two integrals in the latter formula do not contain any problems as all tangential coordinates and parts of metric are smooth, so we will not deal with them, we restrict ourselves only on considerations of the last two integrals which contain normal to $\gamma$ derivatives, that have jumps. By integration by parts, we obtain special conditions for the function $u$ to be a weak solution of $\Delta$ on $\mathcal{M}$, assuming that boundary terms on $\partial \mathcal{M}$ vanish due to the Dirichlet boundary conditions.

$$
I_{2}:=\int_{\Omega_{-}}\left(\partial_{\sigma}\left(\sqrt{g^{-}} \partial_{\sigma} u\right)\right) v(q, \sigma) d q d \sigma=\int_{C l \Omega_{-}} u \Delta v d q d \sigma
$$

here $C l \Omega$ is a notation for the closure of $\Omega$, i.e. we consider the chamber with its included piece-wise smooth boundary. Let us show that the latter is valid:

$$
\begin{gathered}
I_{2}=\left.\int_{\gamma} \sqrt{g^{-}} \partial_{\sigma} u\right|_{\sigma=0} v d q-\left.\int_{\gamma} u \partial_{\sigma} v\right|_{\gamma_{-}} \sqrt{g^{-}} d q \\
\quad+\iint_{\gamma} u\left(\partial_{\sigma} \sqrt{g^{-}} \partial_{\sigma} v\right) d q d \sigma
\end{gathered}
$$

We gave the same formulae for the domain $\Omega_{+}$, thus for the boundary terms to vanish, we have to require some special interface boundary conditions to be satisfied for all function from the domain of our Laplacian. 
Notation 2.18 (Interface Continuity Conditions) We call the following conditions [47] the interface $\gamma$ continuity conditions when they are valid on the interface $\gamma$

$$
\left.[f]\right|_{\gamma}=\left.\left[\sqrt{g} \partial_{\sigma} f\right]\right|_{\gamma}=0
$$

Notation 2.19 (Function jump) Here $[f]$ is a special notation of a function jump on a smooth interface $\gamma$ between two smooth compact areas $\Omega_{-}$and $\Omega_{+}$, i.e.

$$
\left.[f]\right|_{\gamma}=\left.f^{-}\right|_{\gamma}-\left.f^{+}\right|_{\gamma}
$$

Thus we have shown, that the Laplace-Beltrami operator introduced above is selfadjoint when it is determined in the following domain, its closure is called the Dirichlet Laplace-Beltrami operator and will be denoted by $\Delta_{g}$ :

Notation 2.20 (Domain of the Laplacian) We denote the domain of the Dirichlet Laplace-Beltrami operator (2.24) on the admissible Riemannian polyhedron $\mathcal{M}$ by

$$
\mathcal{D}\left(\Delta_{g}(\mathcal{M})\right)=\left\{f \in H^{1}\left(\Omega_{i}\right):[f]_{\gamma}=0,\left.f\right|_{\Gamma_{0}}=0,\left.f\right|_{\gamma} \in H^{1 / 2}(\gamma)\right\}
$$

or, equivalently, for $\mathcal{M}=\Omega_{-} \cup \Omega_{+}, \gamma \subset \Omega_{-} \cup \Omega_{+}$: we have

$$
\begin{gathered}
\mathcal{D}\left(\Delta_{g}(\mathcal{M})\right)=\mathcal{D}\left(\Delta_{ \pm}\right)= \\
\left\{f^{ \pm}=H_{0}^{1}\left(\Omega_{ \pm}\right):\left.f^{+}\right|_{\gamma}=\left.f^{-}\right|_{\gamma},\left.\sqrt{g^{+}} \frac{\partial f^{+}}{\partial \sigma^{+}}\right|_{\gamma}=\left.\sqrt{g^{-}} \frac{\partial f^{-}}{\partial \sigma^{-}}\right|_{\gamma},\left.f\right|_{\Gamma_{0}}=0\right\} .
\end{gathered}
$$

Notation 2.21 (Wave operator) We have one more useful notation:

$$
\square_{g}=\frac{\partial^{2}}{\partial t^{2}}-\Delta_{g}, \text { which is a D'Alembert wave operator. }
$$




\subsection{Spectral problem}

We state a spectral Dirichlet problem, supplying the operator with continuity conditions on the interface and with boundary conditions such that this problem will have a unique solution (see [46]). Thus, for $\mathcal{M}$

$$
\left\{\begin{array}{l}
-\Delta_{g} \varphi_{k}(\mathbf{x})=\lambda_{k} \varphi_{k}(\mathbf{x}), \varphi_{k} \in \mathcal{D}\left(\Delta_{g}\right), \mathbf{x} \in \mathcal{M}, \\
\left.\varphi_{k}(\mathbf{x})\right|_{\Gamma_{0}}=0
\end{array}\right.
$$

for the problem near the interface we consider $\mathcal{M}=\Omega_{-} \cup \Omega_{+}$, then

$$
\left\{\begin{array}{l}
-\Delta_{ \pm} \varphi_{k}(\mathbf{x})=\lambda_{k} \varphi_{k}(\mathbf{x}), \\
\left.\varphi_{k}\right|_{\left(\Gamma_{0}\right) \backslash \gamma}=0 \text { is the Dirichlet type boundary conditions, } \\
\text { continuity conditions of the function and its normal derivative } \\
\text { on the interface, i.e. interface continuity conditions } \gamma,
\end{array}\right.
$$

where $\varphi_{k}(\cdot, \cdot)=\left\{\begin{array}{l}\varphi_{k}^{+},\left(\mathbf{q}, \sigma^{+}\right) \in \Omega_{+}, \\ \varphi_{k}^{-},\left(\mathbf{q}, \sigma^{-}\right) \in \Omega_{-},\end{array}\right.$are the global eigenfunctions of the operator considered on $\mathcal{M}=\Omega_{-} \bigcup \Omega_{+}$, and $\lambda_{k}$ are the corresponding eigenvalues. As above, for the uniqueness of the solution of this problem we supplied this equation with additional interface (continuity of the normal derivative, continuity) conditions.

Remark 2.5.1 Without a loss of generality, we assume that we have chosen starting open part of the boundary $\Gamma_{0}$ such that it does not intersect any singular point $\mathcal{M}$, or, say, $\gamma$.

The standard technique of the spectral theory of elliptic operators ([3], [14], [16], [26], [27], [53]) implies that there exists an orthogonal basis $\left\{\varphi_{k}\right\}_{k=1}^{\infty}$ in $L^{2}(\mathcal{M})$ s.t. each $\varphi_{k}$ is a weak eigenfunction of $\Delta$ in $\mathcal{M}$ with an eigenvalue $\lambda_{k}=\lambda_{k}(\mathcal{M})$, and

$$
0 \leq \lambda_{1} \leq \lambda_{2} \leq \ldots \lambda_{k} \underset{k \rightarrow \infty}{\longrightarrow} \infty
$$


Consider the properties of the eigenfunctions of our problem (2.30), or (2.31). Our metric tensor is $C^{\infty}$ smooth up to the boundary inside each $\Omega^{i n t}$, it satisfies the elliptic regularity property as well, see (2.8). We want to use the fact, that the eigenfunctions $\varphi_{k}(\mathrm{x})$ specify points on $\mathcal{M}$, i.e. that if

$$
\varphi_{k}\left(x_{1}\right)=\varphi_{k}\left(x_{2}\right), \text { for }, k=1,2, \ldots ., \text { then } x_{1}=x_{2},
$$

and then $\varphi_{k}$ form a basis in $L_{2}(\mathcal{M})$. These results can be found in the following books, [46]; [59], [60]. The ruff idea is to use the fact that $H^{1}$ can be compactly embedded into $L^{2}$.

The following result is proved by O. Ladyzhenskaya, see [46].

Lemma 2.5 If domain $\mathcal{M}$ has a piece-wise smooth boundary $\partial \mathcal{M}$, then a bounded set from $H^{1}(\mathcal{M})$ is compact in $L_{2}(\partial \mathcal{M})$.

Consider $u(x) \in \mathcal{D}(\Delta(\mathcal{M}))$ and consider $\gamma$ is a subset of some interface surface. Consider $\gamma$ such that $\mathbf{q}^{\alpha} \in \gamma$ are some smooth coordinates, $\sigma=0$ for $\gamma$. Consider a cylinder

$$
Q_{\delta}:=Q_{\delta}(\gamma)=\left\{\mathbf{x}: 0<\sigma<\delta, \mathbf{q}^{\alpha} \in \gamma\right\} \in \mathcal{M}
$$

For $u(\mathrm{x}) \in \mathcal{D}(\Delta(\mathcal{M}))$ we can construct a sequence of smooth functions $\left\{u^{(m)}(\mathrm{x})\right\}$ such that it converges in $L_{2}\left(Q_{\delta}\right)$ to $u(\mathbf{x})$ and such that $u_{x_{i}}^{(m)}$ converges to $u_{x_{i}}$ in $L_{2}\left(Q_{\delta}\right)$. Consider

$$
u\left(q^{\alpha}, \sigma\right)-u\left(q^{\alpha}, 0\right)=\int_{0}^{\sigma} \frac{\partial u\left(q^{\alpha}, \tau\right)}{\partial \tau} d \tau
$$

for $u \in \mathcal{D}\left(\Delta\left(Q_{\delta}\right)\right)$, then integrating over $\gamma$ and taking squares of both parts, we have

$$
\left\|u\left(q^{\alpha}, \sigma\right)-u\left(q^{\alpha}, 0\right)\right\|_{L_{2}, \gamma}^{2}=\iint_{\gamma}\left(\int_{0}^{\sigma} \frac{\partial u}{\partial \tau} d \tau\right)^{2} d q^{\alpha} \leq \sigma \int_{0}^{\sigma} \int_{\gamma}\left(\frac{\partial u}{\partial \sigma}\right)^{2} d x
$$

and also (see [46]) we have the following estimate:

$$
\int_{\gamma} u^{2}\left(q^{\alpha}, 0\right) d q^{\alpha} \leq \frac{2}{\delta} \int_{Q_{\delta}(\gamma)} u^{2}(x) d x+\delta \int_{Q_{\delta}(\gamma)} u_{\sigma}^{2} d x
$$


Lemma 2.6 (Traces of functions on the interfaces) The consequences of the latter estimates are (see [46]):

(i) functions $u^{(m)}(x)$ converge in $L_{2}(\gamma)$;

(ii) the function which is defined on $\gamma$ as a limit of $u^{(m)}(x)$ in $L_{2}(\gamma)$ is naturally called the trace of $u(x)$ on $\gamma$;

(iii) the traces of $u\left(\boldsymbol{q}^{\alpha}, \sigma\right)$ on $\gamma$ are elements of $L_{2}(\gamma)$ and depend continuously on the parameter $\sigma \in[0, \delta]$;

(iv) the traces of the element $u(x) \in H^{1}(\mathcal{M})$, determined on $\gamma$ as an element of $L_{2}(\gamma)$, does not depend on the choice of the sequences of smooth functions $\left\{u^{(m)}(x)\right\}$.

Remark 2.5.2 (Extra Information) (see [25], [59]) For any interface $\gamma \subset \mathcal{M}$, the continuous trace map $\left.u \mapsto u\right|_{\gamma}: \operatorname{Lip}^{1,2}(\mathcal{M}) \rightarrow L_{2}(\gamma)$ and that map extends uniquely to a continuous map $W^{1,2}=H^{1}(\mathcal{M}) \rightarrow L_{2}(\gamma)$, likewise called trace map, the trace map extends continuously to a a map $T: H^{1}(\Omega) \rightarrow H^{1 / 2}(\gamma)$.

Remark 2.5.3 (Cauchy sequences) There exists function $u^{(m)}(\mathrm{x}) \in C^{\infty}(\mathcal{M})$, converging to $u(\mathbf{x})$ in $H^{1}\left(\mathcal{M}^{i n t}\right),\left\|T u^{(m)}-T u^{(l)}\right\|_{L_{2}}(\partial \mathcal{M}) \leq C\left\|u^{(m)}-u^{(l)}\right\|_{H^{1}\left(\mathcal{M}^{i n t}\right)}$, so that $\left\{T u^{(m)}\right\}_{m=1}^{\infty}$ is a Cauchy sequence in $L_{2}(\partial \mathcal{M})$. Define $T u:=\lim _{m \rightarrow \infty} T u^{(m)}$, the limit taken in $L_{2}(\partial \mathcal{M})$, according to considerations from [46], [27] (the boundary $\partial \mathcal{M}$ can be considered as a finite union of $\Gamma_{i}$ and $\gamma_{j}$ ) this definition does not depend on the particular choice of smooth functions approximation $u$. 


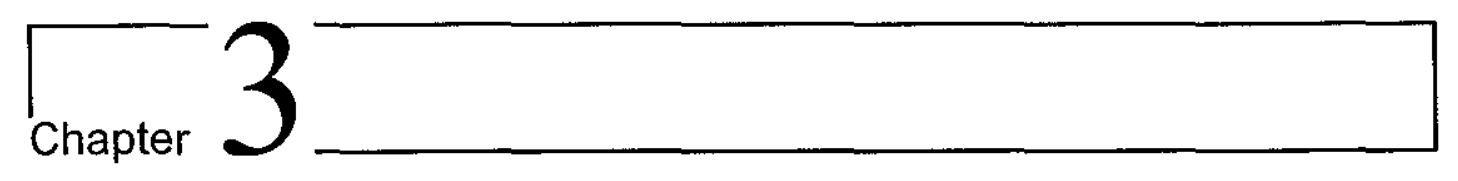

\section{Gaussian Beams near the Interface}

Consider two anisotropic media $\Omega_{-}$and $\Omega_{+}$with a common part which we call the common interface $\gamma$. Assume that the dimension of $\Omega_{ \pm}$is $n$, and the interface $\gamma$ is a hypersurface. Consider $\Omega_{ \pm}$to be $C^{\infty}-$ smooth up to the boundary $\partial \Omega_{ \pm}$Riemannian manifolds. Denote Riemannian metric tensor on $\Omega_{ \pm}$by $g^{\ddagger}$, i.e. $g^{ \pm}=g\left(\Omega_{t}\right)$. We suppose that

$$
\left.g^{-}\right|_{\gamma} \neq\left. g^{+}\right|_{\gamma}
$$

Without a loss of generality we assume that the solution during time $t_{0}>0$ won't leave the domain of regularity of semi-geodesic coordinates and won't reach the manifold boundary $\partial \Omega_{+} \bigcup \partial \Omega_{-}$. The present Chapter contains the description of basic Gaussian beams techniques ([6], [38], [36], [48]) and results from paper [41] for the anisotropic media with an interface.

\subsection{Gaussian beams - "quasiphotons". Definitions}

We seek the solution to the wave equation in the form of a Gaussian beam reviewing the well-known procedure from papers of Babich V., Ulin V., [6], Kachalov A., [36], 


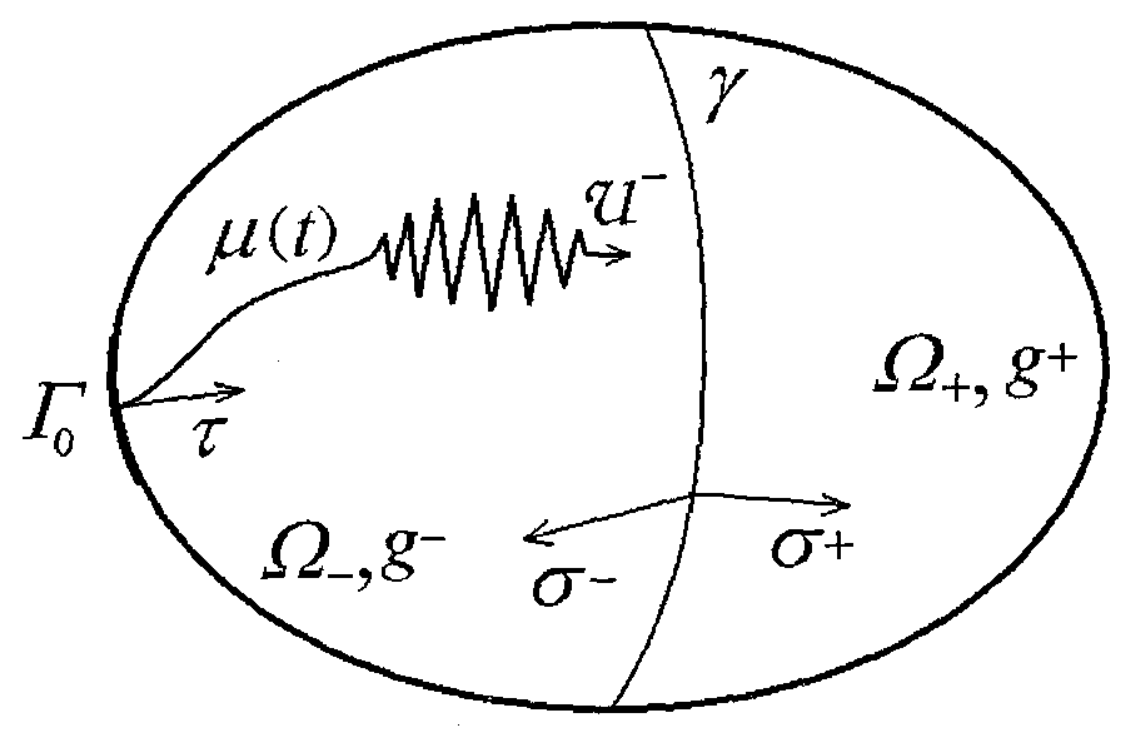

Figure 3.1: Incident Gaussian beam and the interface

Ralston J., [48] and others. Gaussian beam is a complex-valued asymptotic solution to the wave equation such that

$\checkmark$ starting moving from the point on the boundary in some direction, the Gaussian beam is then concentrated at time $t$ near the point $\mu(t)$ on the geodesic determined by the same starting point and same direction. In other words, Gaussian beam decays fast on increasing the distance from that point,

$\checkmark$ it propagates with unit velocity along the geodesic $\mu(t)$ (see Figure 3.1).

Such a solution can be obtained as a unique solution of the initial-boundary value problem for the wave equation, assuming that the source $f^{0}(\varepsilon ; t, \mathbf{z}), \mathbf{z} \in \Gamma_{0}$ is located 
on the boundary $\Gamma_{0}$ in the vicinity of the initial point $M_{0}\left(\mathbf{z}_{0}, \tau_{0}\right)$ at time $-t_{0}<$ $0, \mathrm{z}_{0} \in \Gamma_{0}$, where $(z, \tau)$ are semi-geodesic coordinates corresponding to $\Gamma_{0}$, i.e. $\Gamma_{0}:=$ $\{\mathbf{x} \mid \tau(\mathbf{x})=0\}$ and $\left\{\mathrm{z}^{\alpha}\right.$ are some smooth coordinates on $\left.\Gamma_{0}\right\}$. Denote by

$$
U(t, \mathbf{z}, \tau)=\left\{\begin{array}{l}
U^{+}(t, \mathbf{z}, \tau), \sigma>0 \\
U^{-}(t, \mathbf{z}, \tau), \sigma<0
\end{array}\right.
$$

the solution of the following problem

$$
\left\{\begin{array}{l}
\partial_{t}^{2} U-\Delta_{g} U=\square_{g} U=0, \text { in } \mathcal{M} \times\left[-t_{0}, T\right] \\
\left.U\right|_{t=-t_{0}}=\left.\partial_{t} U\right|_{t=-t_{0}}=0, \\
\left.U\right|_{\Gamma_{0}}=f^{0}(\varepsilon ; t, \mathbf{z}),
\end{array}\right.
$$

where $T>t_{0}$. Here

$$
f^{0}(\varepsilon ; t, \mathbf{z}):=M_{\varepsilon} \chi^{0}(t, \mathbf{z}) \exp \left\{i \varepsilon^{-1} \Theta^{0}(t, \mathbf{z})\right\} V^{0}(\mathbf{z})
$$

is a functional class on $\Gamma_{0} \times \mathbb{R}, \chi^{0}$ is a smooth characteristic function in the vicinity of point $\left(-t_{0}, \mathbf{z}_{0}\right)$, where $M_{\varepsilon}:=(\pi \varepsilon)^{-\frac{n}{4}}, V^{0}(\mathbf{z})$ is a given smooth function, $\varepsilon$ is a small parameter, $0<\varepsilon<1$. The amplitude function can be presented by a sum of smooth homogeneous polynomials on the distances $\left(z-z_{0}\right)$ and $\left(t+t_{0}\right)$ with complex coefficients, the phase function has the following form

$$
\Theta^{0}(t, \mathbf{z})=-\left(t+t_{0}\right)+\frac{1}{2}\left(H^{0}\left(\mathbf{z}-\mathbf{z}_{0}\right),\left(\mathbf{z}-\mathbf{z}_{0}\right)\right)+\frac{i}{2}\left(t+t_{0}\right)^{2},
$$

where $(\cdot, \cdot)$ is a euclidian inner product, $\left(H^{0}\right)^{t}=H^{0}, \Im H^{0}>0$. We follow papers of A. Kachalov, [37], [36], and A. Kachalov, Ya. Kurylev, M. Lassas, [38] to introduce the following definitions:

\section{Definition 3.1 (a Finite Gaussian beam of order $N$ )}

A Finite Gaussian beam (Finite Gaussian beam) of order $N$ is a function $U_{N}(\varepsilon ; t, q, \sigma)$ of the following form:

$$
U_{N}(\varepsilon ; t, \boldsymbol{q}, \sigma) \asymp^{N} M_{\varepsilon} \exp \left\{-(i \varepsilon)^{-1} \Theta_{N}(t, \boldsymbol{q}, \sigma)\right\} \sum_{l=0}^{N} u_{l}(t, \boldsymbol{q}, \sigma)(i \varepsilon)^{l},
$$


where phase function $\Theta_{N}(t, q, \sigma)$ satisfies conditions:

$$
\begin{gathered}
\Im \Theta_{N}(t, \mu(t))=0, \quad \mu(t) \text { is a geodesic, } \\
\Im \Theta_{N}(t, \boldsymbol{q}, \sigma) \geq C_{0}(t) \operatorname{dist}^{2}(\mu, \mu(t)), \operatorname{dist}(\mu, \mu(t)) \neq 0,
\end{gathered}
$$

here $C_{0}$ is a continuous positive function. We have the following inequality valid for the beam:

$$
\left|\square_{g} U_{N}(\varepsilon ; t, q, \sigma)\right| \leq C\left(2 t_{0}\right) M_{\varepsilon} \varepsilon^{N}
$$

where $C\left(2 t_{0}\right)$ does not depend on $\varepsilon$,

$$
\xi=\left(\begin{array}{c}
q \\
\sigma
\end{array}\right), \mu(t)=\left(\begin{array}{c}
q(t) \\
\sigma(t)
\end{array}\right), \quad \boldsymbol{Y}=\xi-\mu(t),
$$

where $0<\eta<1 / 6$. The phase and amplitude function Taylors have the form:

$$
\begin{gathered}
\Theta_{N} \asymp \sum_{l \geq 1}^{K(N)} \theta_{l}(t)=\sum_{|\delta| \geq 1}^{K(N)} \frac{\theta_{\delta}(t)}{\delta !}(\mu-\mu(t))^{\delta}, K(N)=\frac{2(N+2-l)}{1-2 \eta}, \\
u_{p}=\sum_{l \geq 1}^{L(N)} u_{p, l}(t)=\sum_{|\delta| \geq 1}^{L(N)} \frac{u_{n, \delta}(t)}{\delta !}(\mu-\mu(t))^{\delta}, L(N)=\frac{2(N+1-p)}{1-2 \eta},
\end{gathered}
$$

at that $\operatorname{dist}(\mu, \mu(t))=\operatorname{dist}((q, \sigma),(q(t), \sigma(t)))$ is the distance in $\Omega_{ \pm}$.

Notation 3.1 (Imaginary part) We denote by $\Im \theta$ the imaginary part of $\theta$.

Remark 3.1.1 Lemma 2.49 from [38] allows us to restrict ourselves on construction of a finite number of terms in phase and amplitude expansions, see (3.10), (3.11).

Remark 3.1.2 The finite Gaussian beam $U_{N}(\varepsilon ; t, \mathbf{q}, \sigma)$ introduced above is concentrated near $\mu(t)$, i.e. $\left\|\left.U_{N}(\varepsilon ; t, \cdot)\right|_{B_{\rho}}\right\|_{L^{2}}=\mathcal{O}(1)$. Finite Gaussian beam decays exponentially

$$
\left\|\left.U_{N}(\varepsilon ; t, \cdot)\right|_{\left(\Omega_{-} \cup \Omega_{+}\right) \backslash B_{p}}\right\|=\mathcal{O}\left(\varepsilon^{p}\right),
$$

for any $p>0$ outside of the ball $B_{\rho}(\mu(t))$ of radius $\rho>0, \rho \sim \varepsilon^{\frac{1}{2}-\eta}$ (i.e. on the distances more than $\mathcal{O}\left(\varepsilon^{1 / 2-\eta}\right), 0<\eta<1 / 6$ from the geodesic $\left.\mu(t)\right)$. 
Notation 3.2 (Formal series equality) We write $\varkappa^{k}$ when there the equality of formal series up to the order $k$ over all powers $\varepsilon, t, q^{1}, . ., q^{n-1}, \sigma$ and their combinations takes place (we do not care about the convergence here); we denote a formal asymptotic (algebraic) expansion by $\asymp^{k}$, i.e. we write $f(t, \mathbf{Y}(t)) \nwarrow^{k} 0$ when $\left.\partial_{Y}^{\delta} f(t, \mathbf{Y}(t))\right|_{\mathbf{Y}(t)=0}=0$, where $|\delta| \leq k$ is a multi-index. If index $k$ is absent then the expansion is true for any $k$.

\section{Definition 3.2 (An impulse, a quadratic form)}

The first terms of the phase expansion $\Theta_{N}$ of the Gaussian beam have special (polynomial) notations

$$
\theta_{1}\left(t, q^{\alpha}, \sigma\right)=p_{\alpha}(t) \boldsymbol{Y}^{\alpha}+p_{n}(t) \boldsymbol{Y}^{n}
$$

where $p(t):=\left(p_{\alpha}(t), p_{n}(t)\right)$ is an impulse of the Gaussian beam and

$$
\theta_{2}\left(t, q^{\alpha}, \sigma\right)=\frac{1}{2} H_{\alpha \beta}(t) \boldsymbol{Y}^{\alpha} \boldsymbol{Y}^{\beta}+H_{\alpha n}(t) \boldsymbol{Y}^{\alpha} \boldsymbol{Y}^{n}+\frac{1}{2} H_{n n}(t)\left(\boldsymbol{Y}^{n}\right)^{2},
$$

where

$$
H(t):==\left(\begin{array}{cc}
H_{\alpha \beta}(t) & H_{\alpha n}(t) \\
H_{n \beta}(t) & H_{n n}(t)
\end{array}\right)
$$

is called the quadratic form which contains the divergence of rays and a form of the beam. Consequently $\theta_{l}\left(t, q^{\alpha}, \sigma\right)$ are homogeneous polynomials of order $l$ with respect to $\boldsymbol{Y}$. We introduce notation for higher order terms of the expansion:

$$
\theta_{l}=\frac{1}{l !}\left(Q_{\alpha_{1} \ldots \alpha_{l}} \boldsymbol{Y}^{\alpha_{1}} \ldots Y^{\alpha_{l}}+C_{l}^{1} Q_{\alpha_{1} \ldots \alpha_{l-1} n} Y^{\alpha_{1}} \ldots Y^{\alpha_{l-1}} \boldsymbol{Y}^{n}+. .\right)
$$

where $C_{l}^{k}$ are Bernoulli coefficients. The terms terms of order $l>2$ are additional (correctional) and are of minor importance comparing to the impulse and quadratic form.

\section{Definition 3.3 (Formal Gaussian beam)}

We introduce a Formal Gaussian beam (Formal Gaussian beam) by formal expansion in $\varepsilon$ :

$$
U(\varepsilon ; t, \boldsymbol{q}, \sigma) \asymp M_{\varepsilon} \exp \left\{-(i \varepsilon)^{-1} \Theta(t, \boldsymbol{q}, \sigma)\right\} \sum_{l=0}^{\infty}(i \varepsilon)^{l} u_{l}(t, \boldsymbol{q}, \sigma),
$$


where

$$
\Theta(t, \boldsymbol{q}, \sigma) \asymp \sum_{l \geq 1} \theta_{l}(t)=\sum_{|\delta| \geq 1} \frac{\theta_{\delta}(t)}{\delta !}(\mu-\mu(t))^{\delta} .
$$

Definition 3.4 (Gaussian beam of order $N$ )

Gaussian beam $\mathcal{U}_{N}$ of order $N$ is a solution to the following problem

$$
\left\{\begin{array}{l}
\partial_{t}^{2} \mathcal{U}_{N}-\Delta_{g} \mathcal{U}_{N}=\square_{g} \mathcal{U}_{N}=0,(t, \boldsymbol{q}, \sigma) \in\left[-t_{0}, t_{0}\right] \times \mathcal{M} \\
\mathcal{U}_{N}\left(-t_{0}, \boldsymbol{q}, \sigma\right)=U_{N}\left(\varepsilon ;-t_{0}, \boldsymbol{q}, \sigma\right) \\
\partial_{t} \mathcal{U}_{N}\left(-t_{0}, \boldsymbol{q}, \sigma\right)=\partial_{t} U_{N}\left(\varepsilon ;-t_{0}, \boldsymbol{q}, \sigma\right) \\
\left.\mathcal{U}_{N}(t, \boldsymbol{q}, \sigma)\right|_{\Gamma_{0}}=\left.U_{N}(\varepsilon ; t, \boldsymbol{q}, \sigma)\right|_{\Gamma_{0}}
\end{array}\right.
$$

We should mention that

$$
\left|\partial_{t}^{j} \partial_{\mathbf{x}}^{\kappa}\left(\mathcal{U}_{N}(t, \mathbf{q}, \sigma)-\chi(t, \mathbf{q}, \sigma) U_{N}(\varepsilon ; t, \mathbf{q}, \sigma)\right)\right| \leq C M_{\varepsilon} \varepsilon^{N-(j+|\kappa|)}, \forall j \geq 0
$$

here $\kappa$ is a multi-index, $\partial_{\mathbf{x}}^{\kappa}$ denotes partial derivative over $n$ spacial coordinates $\mathbf{x}=$ $\left\{\mathrm{q}^{\alpha}, \sigma\right\}, \chi$ is a characteristic function (smooth mollifier on $\mathcal{M} \times R$ ) equal to one in the vicinity of $\mu=\mu(t)$ and equal to zero outside of this vicinity.

\subsubsection{Solution form}

For the wave equation to have unique solution we should supply it with interface continuity conditions on the smooth interface $\gamma$ between $\Omega_{-}$and $\Omega_{+}$, see (2.26) (see e.g. M. Popov, [47]):

$$
\left.\mathcal{U}^{-}\right|_{\gamma}=\left.\mathcal{U}^{+}\right|_{\gamma},\left.\quad \sqrt{g^{-}} \frac{\partial \mathcal{U}^{-}}{\partial \sigma}\right|_{\gamma}=\left.\sqrt{g^{+}} \frac{\partial \mathcal{U}^{+}}{\partial \sigma}\right|_{\gamma}
$$

where $\mathcal{U}^{ \pm}$is the field value in $\Omega_{-}$, and $\Omega_{+}$. We seek a solution in the form of $i n$ cident and reflected waves in the "first" medium $\Omega_{-}$and transmitted wave in the "second" medium $\Omega_{+}$. Denote these waves by $U^{i n}, U^{r e f}, U^{t r}$ correspondingly (see Figure 3.2). Recalling the representation of formal Gaussian beam (3.14) and 


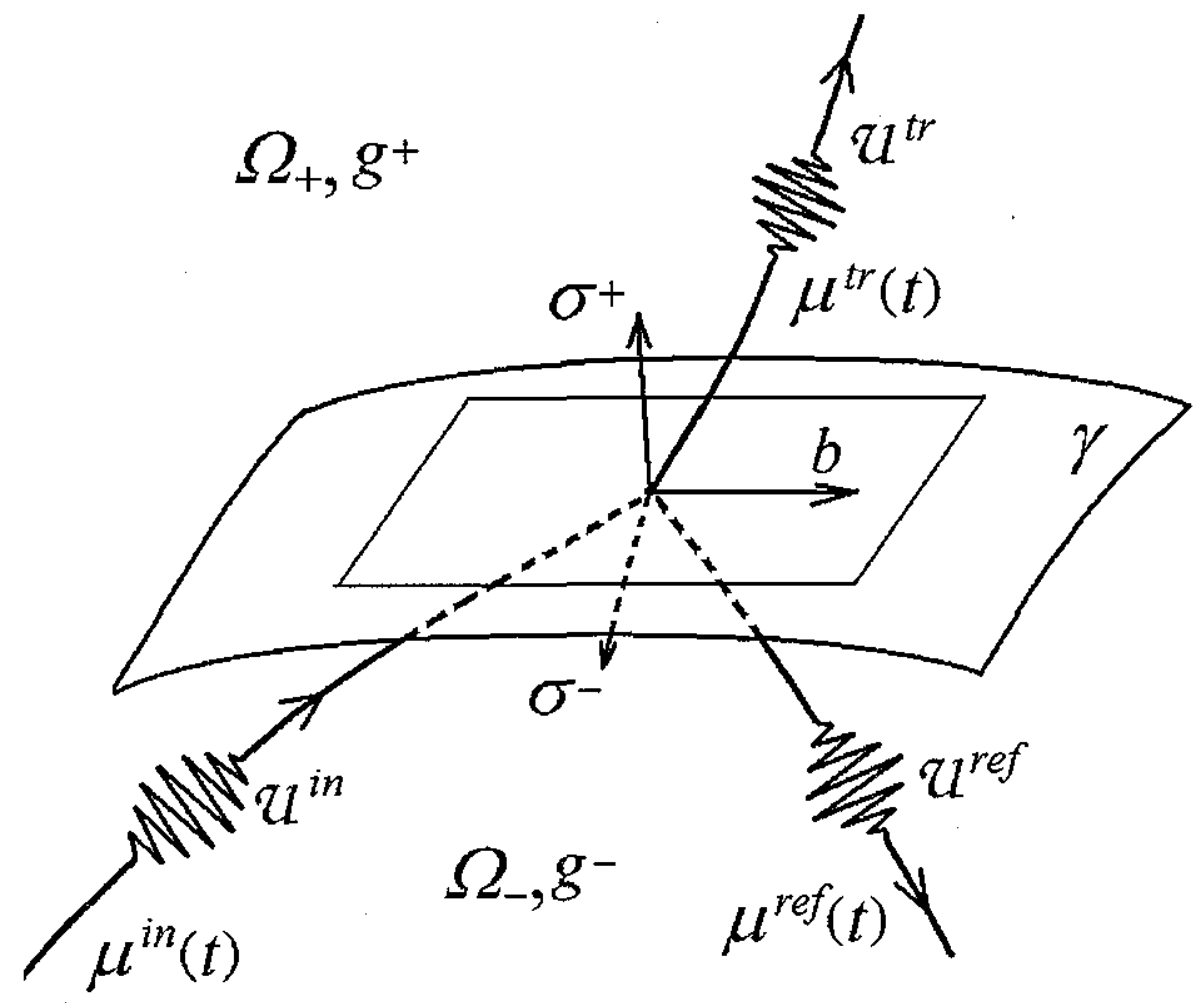

Figure 3.2: Incidence, reflection, transmission Gaussian beams

assuming that it incidents the interface at the origin of semi-geodesic coordinates $M_{1}$ at time $t=0$, we write conditions:

$$
\begin{gathered}
U^{i n}(\varepsilon ; t, \mathbf{q}, 0)+U^{r e f}(\varepsilon ; t, \mathbf{q}, 0) \asymp U^{t r}(\varepsilon ; t, \mathbf{q}, 0) \\
\sqrt{g^{-}}(\mathbf{q}, 0)\left[\frac{\partial U^{i n}}{\partial \sigma^{-}}(\varepsilon ; t, \mathbf{q}, 0)+\frac{\partial U^{r e f}}{\partial \sigma^{-}}(\varepsilon ; t, \mathbf{q}, 0)\right] \asymp \sqrt{g^{+}}(\mathbf{q}, 0) \frac{\partial U^{t r}}{\partial \sigma^{+}}(\varepsilon ; t, \mathbf{q}, 0) .
\end{gathered}
$$

Thus, we write solution in the form:

$$
U^{+}(\varepsilon ; t, \mathbf{q}, \sigma)=U^{t r}(\varepsilon ; t, \mathbf{q}, \sigma), U^{-}(\varepsilon ; t, \mathbf{q}, \sigma)=U^{i n}(\varepsilon ; t, \mathbf{q}, \sigma)+U^{r e f}(\varepsilon ; t, \mathbf{q}, \sigma)
$$




\subsubsection{Main results}

We will construct three formal series $U^{i n}(\varepsilon, \cdot), U^{r e f}(\varepsilon, \cdot), U^{t r}(\varepsilon, \cdot)$ and assume that all three can be smoothly continued into another domain $\Omega_{-} \cup \Omega_{+}$. We truncate these series at order $N$ and construct finite series $U_{N}^{i n}(\varepsilon, \cdot), U_{N}^{r e f}(\varepsilon, \cdot), U_{N}^{t r}(\varepsilon, \cdot)$ in $\Omega_{-}$and $\Omega_{+}$. We will show that these solutions are close to the required solutions to the wave equation (Gaussian beams) in the $H^{1}$-norm. Let $U^{i n}$ propagate in $\Omega_{-}$along the geodesic $\mu^{i n}(t):=\left(q_{i n}^{\alpha}(t), \sigma_{i n}(t)\right)$ and reaches the point $M_{1}=\left(q^{\alpha}, \sigma\right)=(0,0)$ on the interface $\gamma$ at time $t=0$.

Theorem 3.1 (The main Gaussian beams Theorem) Let the formal Gaussian beam $U^{\text {in }}$ (3.14) start movement at time $-t_{0}<0$ at the point $M_{0}=\left(z_{0}, \tau_{0}\right) \in \Gamma_{0}$ and reach the interface $\gamma$ transversally at point $M_{1} \in \gamma$ at time $t=0$. Assume that

$$
0 \leq g_{+}^{\alpha \beta} p_{\alpha}^{i n} p_{\beta}^{i n}<1
$$

Then

1. For $t>0$ the solution to the wave equation can be presented by a sum of two formal Gaussian beams $U^{\text {ref }}$ and $U^{\text {tr }}$; the wave $U^{\text {ref }}$ reflects from the interface and propagates then inside $\Omega_{-}$and $U^{\text {tr }}$ refracts from the interface into $\Omega_{+}$. Both beams $U^{r e f}$ and $U^{\text {tr }}$ can be constructed if the incident beam $U^{\text {in }}$ is known. Constructed by that procedure formal Gaussian beam has all properties to be considered as asymptotic approximation to the required exact solutions $\mathcal{U}^{\text {ref }}, \mathcal{U}^{\text {tr }}$.

2. There are anisotropic analogues of Frenel's and Snell's geometric optics laws for the incident, reflected and transmitted beams. The reflection and transmission angles can be represented in terms of the incidence angle.

3. For any $N$ one can construct the exact solution is a Gaussian beam. This solution $\mathcal{U}^{\text {in }}$ satisfies equation $\square_{g} \mathcal{U}^{\text {in }}=0$ and differs from constructed formal 
Gaussian beam $U_{N}^{i n}+U_{N}^{r e f}$ in $\Omega_{-}$and $U_{N}^{t r}$ in $\Omega_{+}$by function which is small enough with sufficient number of its derivatives. In other words, for any $N$ there exist constants $p(N)$ and $\widetilde{C}_{N}$ such that the difference between the exact solution $\mathcal{U}_{N}$ to the wave equation and formal Gaussian beam is

$$
\left\|\mathcal{U}_{N}-\chi U_{N}\right\|_{C p(N)}\left(\left[-t_{0}, t_{0}\right] ; \mathcal{D}\left(\Delta_{g}\right)\right) \leq \widetilde{C}_{N} \varepsilon^{p(N)}
$$

The first and the second statements of the theorem will be proved constructively in sections $3.2,3.4$, the third statement will be proved in section 3.5 .

Remark 3.1.3 As $g_{\alpha \beta}^{-}(\mathbf{q}, 0) \neq g_{\alpha \beta}^{+}(\mathbf{q}, 0)$, the order of transmitted wave coincides with the order of incident wave. In the case of continuous metric tensor has discontinuous derivative(s), the order of the transmitted field is weaker, but this investigation is beyond the scope of this paper.

Remark 3.1.4 In the case when the condition (3.21) fails we have total internal reflection. The boundary case $g_{+}^{\alpha \beta} p_{\alpha}^{i n} p_{\beta}^{i n}=1$ corresponds to the tangential to the interface direction of the transmitted wave $U^{\text {tr }}$ propagation, we exclude this case from our considerations because the ray expansions fail to be valid as the interface becomes characteristic.

\subsubsection{Formal series}

We follow procedures introduced in papers by V. Babich, V. Ulin, [6], A. Kachalov, [36]. Consider the wave operator $\square_{g}(2.29)$ applied to finite Gaussian beam $U_{N}(\varepsilon ; \cdot)$. For the series to satisfy wave equation asymptotically the phase $\Theta_{N}$ and $u_{l}$ should be the solutions of Hamilton-Jacobi equations and transport equations correspondingly. We omit captions "in, ref, tr" as the following is valid for all three waves. We write out Hamilton-Jacobi equations for the terms of amplitude expansion:

$$
\left[\left(\frac{\partial \Theta_{N}}{\partial t}\right)^{2}-g^{\alpha \beta} \frac{\partial \Theta_{N}}{\partial q^{\alpha}} \frac{\partial \Theta_{N}}{\partial q^{\beta}}-\left(\frac{\partial \Theta_{N}}{\partial \sigma}\right)^{2}\right] \asymp 0,
$$


thus, when they are formally satisfied for $\Theta_{N}$, we write out equations for the amplitude functions. The operator $\mathcal{L}_{\Theta_{N}}$ is called the transport operator:

$$
\mathcal{L}_{\Theta_{N}} u=2 \frac{\partial \Theta_{N}}{\partial t} \frac{\partial u}{\partial t}-2 g^{\alpha \beta} \frac{\partial \Theta_{N}}{\partial q^{\alpha}} \frac{\partial u}{\partial q^{\beta}}-2 \frac{\partial \Theta_{N}}{\partial \sigma} \frac{\partial u}{\partial \sigma}+\left(\square \Theta_{N}\right) \cdot u \text {. }
$$

The first approximation to the amplitude functions follows from

$$
\left[\left(\frac{\partial \Theta_{N}}{\partial t}\right)^{2}-g^{\alpha \beta} \frac{\partial \Theta_{N}}{\partial q^{\alpha}} \frac{\partial \Theta_{N}}{\partial q^{\beta}}-\left(\frac{\partial \Theta_{N}}{\partial \sigma}\right)^{2}\right] u_{0}(t, q, \sigma) \asymp 0 .
$$

Equations for the next amplitude approximations, $l=1, \ldots, N$ take forms of transport equations:

$$
\mathcal{L}_{\Theta_{N}} u_{l} \asymp \square_{g} u_{l-1}, l=0, . ., N, u_{-2}(t, q, \sigma) \equiv u_{-1}(t, q, \sigma) \equiv 0 .
$$

\subsection{Phase functions}

We construct $\Theta_{N}^{r e f}(t, \mathbf{q}, \sigma), \Theta_{N}^{t r}(t, \mathbf{q}, \sigma)$, assuming that they have form (3.10) and that Gaussian beam satisfy wave equation and equate to zero the coefficients corresponding to the same powers of $\varepsilon$ (starting from $\varepsilon^{-2}$ ). We substitute the ansatz (3.14) into the wave equation. Firstly, we will study phase function of the incident field $\Theta_{N}^{i n}(t, \mathbf{q}, \sigma)$. Secondly, we will show how one can obtain finite Gaussian beam $\Theta_{N}^{r e f}(t, \cdot), \Theta_{N}^{t r}(t, \cdot)$ as a series of finite homogeneous polynomials in the terms of the incident field. We will investigate the differential equations, that are satisfied by the terms of those series.

\subsubsection{Main equations}

Consider finite Gaussian beam $U_{N}^{i n}(t, \mathbf{q}, \sigma)$, propagating non-tangetially to the interface $\gamma$ along the geodesic $\mu^{i n}(t):=\left(q_{i n}^{\alpha}(t), \sigma_{i n}(t)\right)$. Following the procedure introduced in, for instance, see [38], consider $\square_{g} U_{N}^{i n}(t, \mathbf{q}, \sigma)$. We have already seen that the phase function must satisfy Hamilton-Jacobi equation (3.22). By construction, the waves must propagate with unit velocity, hence the eikonal equation for $\Theta_{N}^{i n}$ :

$$
g_{-}^{\alpha \beta} \frac{\partial \Theta_{N}^{i n}}{\partial q^{\alpha}} \frac{\partial \Theta_{N}^{i n}}{\partial q^{\beta}}+\left(\frac{\partial \Theta_{N}^{i n}}{\partial \sigma^{-}}\right)^{2} \asymp 1 .
$$


Introduce new notation and rewrite (3.10) (or (3.15)):

$$
\begin{gathered}
\Theta_{N}^{i n}(t, \mathbf{q}, \sigma)=\left(P^{i n}(t)^{t}, \mathbf{Y}^{i n}(t)\right)+\frac{1}{2}\left(H^{i n}(t) \mathbf{Y}^{i n}(t), \mathbf{Y}^{i n}(t)\right)+\ldots, \\
P^{i n}(t)=\left(\begin{array}{lll}
p_{1}^{i n}(t) & \ldots & p_{n-1}^{i n}(t) \\
p_{n}^{i n}(t)
\end{array}\right)^{t}=\left(\begin{array}{c}
p_{\alpha}^{i n}(t) \\
p_{n}^{i n}(t)
\end{array}\right), \\
\mathbf{Y}^{i n}(t)=\xi^{i n}-\mu^{i n}(t)=\left(\begin{array}{c}
q^{1}-q_{i n}^{1}(t) \\
\cdot \\
q^{n-1}-q_{i n}^{n-1}(t) \\
\sigma-\sigma_{i n}(t)
\end{array}\right)=\left(\begin{array}{c}
q^{\alpha}-q_{i n}^{\alpha}(t) \\
\sigma-\sigma_{i n}(t)
\end{array}\right), \\
H^{i n}(t)=\left(\begin{array}{cc}
H_{\alpha \beta}^{i n}(t) & H_{\alpha n}^{i n}(t) \\
H_{n \beta}^{i n}(t) & H_{n n}^{i n}(t)
\end{array}\right),
\end{gathered}
$$

where $\mu(t)$ was introduced in (3.9). Continue standard procedure of Gaussian beams construction (see, for instance, [6], [36]), the eikonal equation (3.26) implies the equation for all terms in expansion (3.10), including Hamilton-Jacobi system of equations (3.34) for impulses (3.33) and Riccati equation (3.35) for the quadratic forms. Denote the hamiltonian of the system by $h$ :

$$
\begin{gathered}
h^{i n}(\mathbf{q}, \sigma ; \mathbf{p})=(\mathbf{p}, \mathbf{p})_{g_{-}}^{1 / 2}=\sqrt{g_{-}^{\alpha \beta} p_{\alpha}^{i n}(t) p_{\beta}^{i n}(t)+\left(p_{n}^{i n}(t)\right)^{2}} \equiv 1 \\
\left(h^{i n}\right)^{2}=g_{-}^{\alpha \beta} \frac{\partial \Theta^{i n}}{\partial q^{\alpha}} \frac{\partial \Theta^{i n}}{\partial q^{\beta}}+\left(\frac{\partial \Theta^{i n}}{\partial \sigma}\right)^{2}=g_{-}^{\alpha \beta} p_{\alpha}^{i n} p_{\beta}^{i n}+\left(p_{n}^{i n}\right)^{2}=\left(\frac{\partial \Theta^{i n}}{\partial t}\right)^{2}
\end{gathered}
$$

then the impulse equation is

$$
g_{-}^{\alpha \beta} p_{\alpha}^{i n}(t) p_{\beta}^{i n}(t)+\left(p_{n}^{i n}(t)\right)^{2} \equiv 1
$$

Hamilton equations (canonical equations) are:

$$
\left\{\begin{array}{l}
\dot{q}_{i n}^{\alpha}(t)=\frac{\partial h^{i n}}{\partial p_{\alpha}}=\frac{1}{h^{i n}} g_{-}^{\alpha \beta} p_{\beta}^{i n}(t), \\
\dot{\sigma}_{i n}(t)=\frac{\partial h^{i n}}{\partial p_{n}}=\frac{1}{h^{i n}} p_{n}^{i n}(t) \\
\dot{p}_{\gamma}^{i n}(t)=-\frac{\partial h^{i n}}{\partial q^{\gamma}}=-\frac{1}{2 h^{i n}} p_{\alpha}^{i n}(t) p_{\beta}^{i n}(t) \frac{\partial g_{-}^{\alpha \beta}}{\partial q^{\gamma}} \\
\dot{p}_{n}^{i n}(t)=-\frac{\partial h^{i n}}{\partial \sigma}=-\frac{1}{2 h^{i n}} p_{\alpha}^{i n}(t) p_{\beta}^{i n}(t) \frac{\partial g_{-}^{\alpha \beta}}{\partial \sigma}
\end{array}\right.
$$


The solution of this system is a bi-characteristic $\left(q_{i n}^{\alpha}(t), \sigma_{i n}(t) ; p_{\alpha}^{i n}(t), p_{n}^{i n}(t)\right)$. The third equation which is satisfied by the quadratic form of the Gaussian beam is the Riccati equation:

$$
\frac{d}{d t} H^{i n}+D^{i n}+\left(B^{i n} H^{i n}+H^{i n}\left(B^{i n}\right)^{t}\right)+H^{i n} C^{i n} H^{i n}=0
$$

where the coefficients $\left(B^{i n}\right)^{t}=B^{i n *},\left(C^{i n}\right)^{t}=C^{i n *}=C,\left(D^{i n}\right)^{t}=D^{i n *}=D$ are $n \times n$ matrices of second derivatives of hamiltonian, taken at point $(\mathbf{q}, \sigma ; \mathbf{p})=$ $\left(\mathbf{q}_{i n}(t), \sigma_{i n}(t) ; \mathbf{p}^{i n}(t)\right)$ on the bi-characteristic:

$$
\begin{gathered}
D^{i n}=\left(\begin{array}{cc}
D_{\alpha \beta}^{i n} & D_{\alpha n}^{i n} \\
D_{n \beta}^{i n} & D_{n n}^{i n}
\end{array}\right)=\left(\begin{array}{cc}
\frac{\partial^{2} h^{i n}}{\partial q^{\alpha} \partial q^{\beta}} & \frac{\partial^{2} h^{i n}}{\partial q^{\alpha} \partial \sigma} \\
\frac{\partial^{2} h^{i n}}{\partial \sigma \partial q^{\beta}} & \frac{\partial^{2} h^{i n}}{\partial \sigma^{2}}
\end{array}\right), \\
B^{i n}=\left(\begin{array}{cc}
B_{\alpha}^{\beta^{i n}} & B_{\alpha}^{n i n} \\
B_{n}^{\beta^{i n}} & B_{n}^{n i n}
\end{array}\right)=\left(\begin{array}{cc}
\frac{\partial^{2} h^{i n}}{\partial q^{\alpha} \partial p_{\beta}} & \frac{\partial^{2} h^{i n}}{\partial q^{\alpha} \partial p_{n}} \\
\frac{\partial^{2} h^{i n}}{\partial q^{\beta} \partial p_{n}} & \frac{\partial^{2} h^{i n}}{\partial \sigma \partial p_{n}}
\end{array}\right), \\
C^{i n}=\left(\begin{array}{cc}
C^{\alpha \beta^{i n}} & C^{\alpha n i n} \\
C^{n \beta^{i n}} & C^{n n i n}
\end{array}\right)=\left(\begin{array}{cc}
\frac{\partial^{2} h^{i n}}{\partial p_{\alpha} \partial p_{\beta}} & \frac{\partial^{2} h^{i n}}{\partial p_{\alpha} \partial p_{n}} \\
\frac{\partial^{2} h^{i n}}{\partial p_{n} \partial p_{\beta}} & \frac{\partial^{2} h^{i n}}{\partial p_{n}^{2}}
\end{array}\right) .
\end{gathered}
$$

Our next step is to obtain equations for the higher order terms. Note that all constructed equations are recurrent because each time the higher order terms of the phase vanish along bi-characteristics. The homogeneous term $\theta_{l}^{i n}$ of the order $l$ in the Tailor's expansion (3.22) depends on $\theta_{m}^{i n}$, where $m \leq l+1$. Recall that terms containing $\theta_{l+1}^{i n}$ equal zero along bi-characteristics. Finally the obtained differential equations for the homogeneous polynomials $\theta_{l}^{i n}, l \geq 3$ are linear (see [38]),

$$
\frac{\partial \theta_{l}^{i n}}{\partial t}+\mathcal{N}_{j}^{(i n) i} \frac{\partial \theta_{l}^{i n}}{\partial\left(\mathbf{Y}^{i n}\right)^{i}}\left(\mathbf{Y}^{i n}\right)^{j}=\mathcal{F}_{l}\left(\theta_{m}^{i n}\right), \quad l=3,4, . ., m<l
$$

The components $\mathcal{N}_{j}^{(t r) i}$ form $n \times n$ matrix:

$$
\mathcal{N}_{j}^{(i n) i}(t)=\frac{\partial^{2} h^{i n}}{\partial x^{i} \partial p_{j}}+\frac{\partial^{2} h^{i n}}{\partial p_{i} \partial p_{k}} H_{k j}^{i n}=\left[B_{i n}^{t}+C_{i n} H_{i n}\right]_{j}^{i},
$$

where matrices $B^{i n}, C^{i n}$ were determined above in (3.37)-(3.38). 
Remark 3.2.1 The obtained equations (3.39) can be reduced to the linear ODE with respect to $t$ for $\theta_{l}^{i n}(t)$. They require initial data to be given for the uniqueness of their solutions. Note once again that those equations obtained for the incident field can be written formally for the reflected and for the transmitted fields, as we used only general properties of Gaussian beams on obtaining them, as we equal coefficients of the homogeneous polynomials considered.

\subsubsection{Required preparations}

Consider phase function expansion as a series (3.27) and re-expand it in the vicinity of $t=0$, i.e. we expand it into Tailor's series $P^{i n}(t)(3.28), H^{i n}(t)(3.30)$ and $\mathbf{Y}^{i n}(t)$ (3.29) and construct new forms, now with respect to $n+1$ variables $t, \mathbf{q}, \sigma$. Denote these new forms by $\widetilde{P}^{i n}, \widetilde{H}^{\text {in }}$, i.e. tilde above the notation means it is a coefficient of the new form, obtained as a result of the expansion:

$$
\begin{array}{r}
\mathbf{Y}^{i n}(t) \asymp\left(\begin{array}{c}
q^{\alpha}-\dot{q}_{i n}^{\alpha}(0) t-\frac{1}{2} \ddot{q}_{i n}^{\alpha}(0) t^{2}+\ldots \\
\sigma-\dot{\sigma}_{i n}(0) t-\frac{1}{2} \ddot{\sigma}_{i n}(0) t^{2}+\ldots
\end{array}\right), \\
\Theta_{N}^{i n}(t, \mathbf{q}, \sigma) \asymp^{2} \widetilde{p}_{0} t+\widetilde{p}_{\alpha}^{i n} q^{\alpha}+\widetilde{p}_{n}^{i n} \sigma+\frac{1}{2} \widetilde{H}_{00}^{i n} t^{2} \\
+\frac{1}{2} \widetilde{H}_{\alpha \beta}^{i n} q^{\alpha} q^{\beta}+\frac{1}{2} \widetilde{H}_{n n}^{i n} \sigma^{2}+\widetilde{H}_{0 \alpha}^{i n} t q^{\alpha}+\widetilde{H}_{0 n}^{i n} t \sigma+\widetilde{H}_{\alpha n}^{i n} q^{\alpha} \sigma \\
=\left(\left(\widetilde{\mathbf{P}}^{i n}\right)^{t},\left(\begin{array}{c}
t \\
q^{\alpha} \\
\sigma
\end{array}\right)\right)+\frac{1}{2}\left(\widetilde{\mathbf{H}}^{i n}\left(\begin{array}{c}
t \\
q^{\alpha} \\
\sigma
\end{array}\right),\left(\begin{array}{c}
t \\
q^{\alpha} \\
\sigma
\end{array}\right)\right),
\end{array}
$$

where

$$
\widetilde{\mathbf{H}}^{i n}=\left(\begin{array}{ccc}
\widetilde{H}_{00}^{i n} & \widetilde{H}_{0 \beta}^{i n} & \widetilde{H}_{0 n}^{i n} \\
\widetilde{H}_{\alpha 0}^{i n} & \widetilde{H}_{\alpha \beta}^{i n} & \widetilde{H}_{\alpha n}^{i n} \\
\widetilde{H}_{n 0}^{i n} & \widetilde{H}_{n \beta}^{i n} & \widetilde{H}_{n n}^{i n}
\end{array}\right), \widetilde{\mathbf{P}}^{i n}=\left(\begin{array}{c}
\widetilde{p}_{0}^{i n} \\
\widetilde{p}_{\alpha}^{i n} \\
\widetilde{p}_{n}^{i n}
\end{array}\right)=\left(\begin{array}{c}
-1 \\
p_{\alpha}^{i n}(0) \\
p_{n}^{i n}(0)
\end{array}\right),
$$




$$
\left\{\begin{array}{l}
\frac{1}{2} \widetilde{H}_{00}^{i n}=-\dot{p}_{\alpha}^{i n}(0) \dot{q}_{i n}^{\alpha}(0)-\dot{p}_{n}^{i n}(0) \dot{\sigma}_{i n}(0)-\frac{1}{2}\left(p_{\alpha}^{i n}(0) \ddot{q}_{i n}^{\alpha}(0)+p_{n}^{i n}(0) \ddot{\sigma}_{i n}(0)\right) \\
\quad+\frac{1}{2} H_{n n}^{i n}(0)\left(\dot{\sigma}_{i n}(0)\right)^{2}+\frac{1}{2} H_{\alpha \beta}^{i n}(0) \dot{q}_{i n}^{\alpha}(0) \dot{q}_{i n}^{\beta}(0)+H_{n \alpha}^{i n}(0) \dot{q}_{i n}^{\alpha}(0) \dot{\sigma}_{i n}(0) \\
\widetilde{H}_{\alpha \beta}^{i n}=H_{\alpha \beta}^{i n}(0) \\
\widetilde{H}_{n n}^{i n}=H_{n n}^{i n}(0) \\
\widetilde{H}_{0 \alpha}^{i n}=\dot{p}_{\alpha}^{i n}(0)-H_{\alpha \beta}^{i n}(0) \dot{q}_{i n}^{\beta}(0)-H_{\alpha n}^{i n}(0) \dot{\sigma}_{i n}(0) \\
\widetilde{H}_{n 0}^{i n}=\dot{p}_{n}^{i n}(0)-H_{n n}^{i n}(0) \dot{\sigma}_{i n}(0)-H_{\alpha n}^{i n}(0) \dot{q}_{i n}^{\alpha}(0) \\
\widetilde{H}_{\alpha n}^{i n}=H_{\alpha n}^{i n}(0)
\end{array}\right.
$$

The obtained expansion coefficients will be helpful in finding the initial data for the quadratic form. Note that similar expansions can be presented also for the reflected and transmitted fields.

\subsubsection{Impulses $\mathbf{p}^{r e f}, \mathbf{p}^{\text {tr }}$ construction}

The goal of this section is to construct the first linear terms in expansion of the reflected and transmitted phase functions $\Theta_{N}^{r e f}$ and $\Theta_{N}^{t r}$, i.e. to construct impulses $P^{r e f}(t), P^{t r}(t)$ (similar to (3.28)). We will find impulses by given initial point and unit velocity in metric.

Consider expansion (3.12) with respect to $t, \mathbf{q}, \sigma$ (we omit similar expansion $\theta_{1}^{t r}(t)$ as it has similar form):

$$
\theta_{1}^{r e f}(t, \mathbf{q}, \sigma)=p_{\alpha}^{r e f}(0) q^{\alpha}+p_{n}^{r e f}(0) \sigma-p_{\alpha}^{r e f}(0) \dot{q}_{r e f}^{\alpha}(0) t-p_{n}^{r e f}(0) \dot{\sigma}_{r e f}(0) t .
$$

We want to find initial data using interface data (3.19) to this end, the first continuity condition implies equality

$$
\theta_{1}^{\text {in }}(t, \mathbf{q}, 0)=\theta_{1}^{r e f}(t, \mathbf{q}, 0)=\theta_{1}^{t r}(t, \mathbf{q}, 0)
$$

hence it is clear that tangential components of impulses (corresponding to $\gamma$ ) are equal to

$$
p_{\alpha}^{i n}(t)=p_{\alpha}^{r e f}(t)=p_{\alpha}^{t r}(t), \Rightarrow p_{\alpha}^{i n}(0)=p_{\alpha}^{r e f}(0)=p_{\alpha}^{t r}(0)
$$


Then assuming that the value of the incident field of the Gaussian beam is given at $t=0$, i.e. assuming that we know $P^{i n}(0)$ and recalling that the velocity is unit, i.e. $\left|P^{t r}(t)\right|_{g_{+}}=1,\left|P^{r e f}(t)\right|_{g_{-}}=1$ (impulse satisfies eikonal equation (3.26) for any $t$, thus for $t=0$ ), we write

$$
\begin{gathered}
\left|P^{t r}(0)\right|=h\left(0,0 ; P^{t r}(0)\right)= \pm \sqrt{g_{+}^{\alpha \beta}(0,0) p_{\alpha}^{i n}(0) p_{\beta}^{i n}(0)+\left(p_{n}^{t r}(0)\right)^{2}}=1 \\
\left|P^{r e f}(0)\right|=h\left(0,0 ; P^{r e f}(0)\right)= \pm \sqrt{g_{-}^{\alpha \beta}(0,0) p_{\alpha}^{i n}(0) p_{\beta}^{i n}(0)+\left(p_{n}^{r e f}(0)\right)^{2}}=1 .
\end{gathered}
$$

Then both $p_{n}^{\text {tr }}(0)$ and $p_{n}^{\text {ref }}(0)$ can be determined up to sign of the square root. As the transmitted Gaussian beam propagates inside $\Omega_{+}$we should take $\frac{\partial \theta^{t r}}{\partial \sigma^{+}}>0$, i.e. $p_{n}^{t r}(0)>0$, the reflected beam propagates inside $\Omega_{-}$, thus $p_{n}^{i n}(0)=-p_{n}^{\text {ref }}(0)$, i.e.

$$
\begin{gathered}
p_{n}^{t r}(0)=\sqrt{1-g_{+}^{\alpha \beta}(0,0) p_{\alpha}^{i n}(0) p_{\beta}^{i n}(0)}>0, \\
p_{n}^{r e f}(0)=\sqrt{1-g_{-}^{\alpha \beta}(0,0) p_{\alpha}^{i n}(0) p_{\beta}^{i n}(0)}=-p_{n}^{i n}(0)>0 .
\end{gathered}
$$

Remark 3.2.2 Condition $\left|p^{t r}\right|<1$ guarantees that we do not have total internal reflection case.

We found initial values $P^{r e f}(0)$ (and $P^{t r}(0)$ ). The initial point $\left(\mathrm{q}_{t r}(0), \sigma_{t r}(0)\right)=$ $\left(\mathbf{q}_{r e f}(0), \sigma_{r e f}(0)\right)=(0,0)$ of the corresponding bi-characteristics $\left(\mathbf{q}_{t r}(t), \sigma_{t r}(t) ; \mathbf{p}_{t r}(t)\right)$ and $\left(\mathbf{q}_{r e f}(t), \sigma_{r e f^{\prime}}(t) ; \mathbf{p}_{r e f}(t)\right)$ is known, thus we can solve Hamilton system of equations (3.34). The corresponding hamiltonians are respectively,

$$
\begin{gathered}
\left(h^{t r}\right)^{2}=g_{+}^{\alpha \beta}(\mathbf{q}, \sigma) p_{\alpha}^{t r}(t) p_{\beta}^{t r}(t)+\left(p_{n}^{t r}(t)\right)^{2}=1 . \\
\left(h^{r e f}\right)^{2}=g_{-}^{\alpha \beta}(\mathbf{q}, \sigma) p_{\alpha}^{r e f}(t) p_{\beta}^{r e f}(t)+\left(p_{n}^{r e f}(t)\right)^{2}=1 .
\end{gathered}
$$




\subsubsection{Quadratic forms}

The goal of this section is to construct the second (quadratic) terms of the phase function expansion (3.27), namely, $H^{r e f}(t, \cdot), H^{t r}(t, \cdot)$ in assumption that $H^{\text {in }}(0)$ is given and that we have already constructed impulses. To this end we have to solve Riccati equation (3.35) after calculation initial data $H^{t r}(t=0)$ and $H^{\text {ref }}(t=0)$.

Initial data $\theta_{2}^{r e f}(0, \mathbf{q}, \sigma), \theta_{2}^{t r}(0, \mathbf{q}, \sigma)$.

The goal of this subsection is to construct initial data with required properties using interface boundary data (given on $\gamma$ ), i.e. given $\widetilde{H}_{0 \alpha}^{i n}, \widetilde{H}_{\alpha \beta}^{i n}, \widetilde{H}_{00}^{i n}$, and assuming that we have already constructed impulses and found geodesics we have to express $H_{\alpha \beta}^{\text {ref,tr }}(t=$ $0), H_{\alpha n}^{r e f, t r}(t=0), H_{n n}^{r e f, t r}(t=0)$ in terms of known values. This time we also use continuity conditions (3.19) recalling that $\left.\Theta^{i n}\right|_{\gamma}=\left.\Theta^{t r}\right|_{\gamma}=\left.\Theta^{r e f}\right|_{\gamma}$, see, for instance, [6]. The latter we rewrite as

$$
\widetilde{H}_{\alpha \beta}^{i n}=\widetilde{H}_{\alpha \beta}^{r e f}=\widetilde{H}_{\alpha \beta}^{t r}=H_{\alpha \beta}^{i n}(0)=H_{\alpha \beta}^{r e f}(0)=H_{\alpha \beta}^{t r}(0)
$$

Then we get on the interface $\sigma=0(3.41)$

$$
\begin{array}{r}
-t+p_{\alpha}^{i n} q^{\alpha}+\frac{1}{2} \widetilde{H}_{00}^{i n} t^{2}+\frac{1}{2} \widetilde{H}_{\alpha \beta}^{i n} q^{\alpha} q^{\beta}+\widetilde{H}_{0 \alpha}^{i n} t q^{\alpha}+\ldots \\
=-t+p_{\alpha}^{r e f} q^{\alpha}+\frac{1}{2} \widetilde{H}_{00}^{r e f} t^{2}+\frac{1}{2} \widetilde{H}_{\alpha \beta}^{r e f} q^{\alpha} q^{\beta}+\widetilde{H}_{0 \alpha}^{r e f} t q^{\alpha}+\ldots
\end{array}
$$

Consequently,

$$
\left\{\begin{array}{l}
\widetilde{H}_{00}^{i n}=\widetilde{H}_{00}^{r e f}=\widetilde{H}_{00}^{t r} \\
\widetilde{H}_{0 \alpha}^{i n}=\widetilde{H}_{0 \alpha}^{r e f}=\widetilde{H}_{0 \alpha}^{t r} .
\end{array}\right.
$$

Now we substitute coefficients (3.43) and similar expression for $H^{r e f}$ and $H^{t r}$ into the second equation (3.50). Thus we get the representations for $H_{\alpha n}^{r e f, t r}(0)$ in terms of the given incident field and known derivatives of the geodesics:

$$
H_{\alpha n}^{r e f}(0)=-H_{\alpha n}^{i n}(0)
$$


We will not present here formulae for reflected and transmitted quadratic forms at time zero as they are massive, we present only expression of their imaginary parts as we will need them later. We obtain following for the reflected field

$$
\Im H_{\alpha n}^{t r}(0) \dot{\sigma}_{t r}(0)=-\Im H_{\alpha n}^{i n}(0) \dot{\sigma}_{i n}(0)-\Im H_{\alpha \beta}^{i n}(0)\left[\dot{q}_{i n}^{\alpha}(0)-\dot{q}_{t r}^{\alpha}(0)\right]
$$

here we should recall, that $\Im$ is a notation for the imaginary part. Similarly $H_{n n}^{r e f}(t=$ $0)$ :

$$
\begin{gathered}
\Im\left(H_{n n}^{r e f}(0)\left(\dot{\sigma}_{r e f}(0)\right)^{2}\right)=\Im\left(H_{n n}^{i n}(0)\left(\dot{\sigma}_{i n}(0)\right)^{2}\right) \\
\Im\left(H_{n n}^{t r}(0)\left(\dot{\sigma}_{t r}(0)\right)^{2}\right)=-2 \Im\left(H_{n \alpha}^{t r}(0) \dot{q}_{t r}^{\alpha}(0) \dot{\sigma}_{t r}(0)+H_{n \alpha}^{i n}(0) \dot{q}_{i n}^{\alpha}(0) \dot{\sigma}_{i n}(0)\right) \\
+\Im\left(H_{n n}^{i n}(0)\left(\dot{\sigma}_{i n}(0)\right)^{2}+H_{\alpha \beta}^{i n}(0)\left[\dot{q}_{t r}^{\alpha}(0) \dot{q}_{t r}^{\beta}(0)-\dot{q}_{i n}^{\alpha}(0) \dot{q}_{i n}^{\beta}(0)\right]\right)
\end{gathered}
$$

Thus, we found initial data for the reflected and transmitted quadratic forms, using only given incident field and recently constructed impulses.

\section{Initial boundary-value problem.}

As we have already constructed initial data $H^{r e f}(0), H^{\operatorname{tr}}(0)$, we can start solving Riccati equation (3.35). If we succeed to show that initial quadratic form $H(0)=H_{0}$ satisfies next lemma conditions, then the solution to the Riccati equation is required quadratic form of Gaussian beam. The next lemma is Lemma 2.56 from [38] (see also V. Babich, V. Ulin, [6], or V. Babich, V. Buldyrev, I. Molotkov, [7], A. Kachalov, [36]):

Lemma 3.1 (Lemma 2.56 from [38]) Let $H_{0}$ be $n \times n$ complex-valued matrix such that

$$
\begin{aligned}
& H_{0}=H_{0}^{t}, \\
& \Im H_{0}>0 .
\end{aligned}
$$

Then: 
(i) the initial boundary-value problem for the Riccati equation (3.35) with initial values

$$
\left.H\right|_{t=0}=H_{0}
$$

has a unique solution $H(t), t \in \mathbf{R}$. The derivatives are calculated at the point

$$
(q, \sigma ; p)=(q(t), \sigma(t) ; p(t))
$$

i.e. on the bi-characteristic, which is a solution to the Hamilton equation (3.34).

(ii) The solution $H(t), t \in \mathbf{R}$ is symmetric $H(t)=H(t)^{t}$, and $\Im H(t)>0$.

(iii) Besides that for any $Y_{0}, Z_{0}$ such that $H_{0}=Z_{0} Y_{0}^{-1}$, matrix $H(t)$ can be represented in the form $H(t)=Z(t) Y(t)^{-1}$. The pair of matrices $(Z(t), Y(t))$ is a solution to the initial boundary-value problem,

$$
\begin{array}{cc}
\frac{d}{d t} Y(t)=B^{t} \cdot Y+C \cdot Z, & \left.Y\right|_{t=0}=Y_{0} \\
\frac{d}{d t} Z(t)=-D \cdot Y-B \cdot Z, & \left.Z\right|_{t=0}=Z_{0},
\end{array}
$$

where matrix $Y(t)$ is non-degenerate for any $t \in \mathbf{R}, \operatorname{det} Y(t) \neq 0$.

Lemma 3.2 The determinant $\operatorname{det}(\Im H(t)) \cdot|\operatorname{det} Y(t)|^{2}$ is constant for any Gaussian beam.

Both lemmas are proved in [38].

To use Lemma 2.56 results we need to show that obtained $H^{r e f}(0), H^{\operatorname{tr}}(0)$ satisfy its requirements (they should be symmetric and positive-definite).

Statement 3.2.4.1 Let us assume that we know that $\Im \theta_{2}^{i n}(t, q, 0)>0$. Let $\theta_{2}^{\text {in }}(t, q, 0)$ be symmetric. Then $\Im \theta_{2}^{\text {ref,tr }}(0, q, \sigma)>0$ and $\theta_{2}^{\text {ref,tr }}(0, q, \sigma)$ are also symmetric.

Proof We start the proof of the statement with showing the symmetry of $H^{r e f, t r}(0)$. In fact, we cannot state that homogeneous polynomials $\theta_{2}^{\text {ref,tr }}(0, \mathbf{q}, \sigma)$ are determined by 
symmetric matrices $H^{\text {tr }}(0)$ and $H^{r e f}(0)$, but we can always choose unique symmetric tensor which gives birth to the required polynomial $\theta^{r e f, t r}(0, q, \sigma)$. Let us prove the second part of the statement. Assume we know that imaginary part $\Im H^{r e f, t r}(t)>0$, (3.30) for any $t$ i.e.

$$
\left(\left(\begin{array}{cc}
\Im H_{\alpha \beta}^{i n}(0) & \Im H_{\alpha n}^{i n}(0) \\
\Im H_{n \beta}^{i n} & \Im H_{n n}^{i n}(0)
\end{array}\right)\left(\begin{array}{c}
q^{\alpha} \\
\sigma
\end{array}\right),\left(\begin{array}{c}
q^{\beta} \\
\sigma
\end{array}\right)\right)>0 .
$$

Necessary and sufficient condition of positive definiteness by classical Silvester criteria (see, say, [62] for details) is positiveness of all main co-factors.

Note that all main co-factors of matrices $\Im H^{i n}(0)$, $\Im H^{r e f}(0), \Im H^{t r}(0)$ excluding determinant are equal. Thus in order to show that $\Im H^{r e f}(0), \Im H^{t r}(0)$ is positive-definite one have to investigate the positiveness of their determinants only. Consider firstly the reflected quadratic form

$$
\operatorname{det}\left(\begin{array}{cc}
\Im H_{\alpha \beta}^{r e f}(0) & \Im H_{\alpha n}^{r e f}(0) \\
\Im H_{n \beta}^{r e f}(0) & \Im H_{n n}^{r e f}(0)
\end{array}\right)=\operatorname{det}\left(\begin{array}{cc}
\Im H_{\alpha \beta}^{i n}(0) & -\Im H_{\alpha n}^{i n}(0) \\
-\Im H_{n \beta}^{i n}(0) & \Im H_{n n}^{i n}(0)
\end{array}\right) .
$$

As we see the determinants are equal, and thus we proved that $\Im H^{r e f}(0)>0$. Consider now $\Im H^{\operatorname{tr}}(0)$. Obviously, multiplying firstly the last column and secondly the last row by constant $\dot{\sigma}_{i n}(0), \dot{\sigma}_{t r}(0)$ in matrices $\Im H^{i n}(0), \Im H^{t r}(0)$ correspondingly $((3.52),(3.54))$, the sign of determinant does not change. We work with matrix

$$
\left(\begin{array}{cc}
\Im H_{\alpha \beta}^{i n}(0) & \Im H_{\alpha n}^{i n}(0) \dot{\sigma}_{i n}(0) \\
\Im H_{n \beta}^{t r}(0) \dot{\sigma}_{i n}(0) & \Im H_{n n}^{t r}(0) \dot{\sigma}_{i n}^{2}(0)
\end{array}\right)
$$

its determinant does not change, it is positive. Next we use linear transformations (they do not change the determinant) and get matrix $\Im H^{t r}(0)$ with components ((3.49), (3.52), (3.54)), this will prove its positive-definiteness. The noted above transformation are: we add the linear combination of all right rows with a factor $-\left[\dot{q}_{i n}^{\beta}(0)-\dot{q}_{t r}^{\beta}(0)\right]$ to the last column, then the right column of the obtained matrix has components $-\Im H_{\alpha n}^{i n} \dot{\sigma}_{i n}(0)-\left[\dot{q}_{i n}^{\beta}(0)-\dot{q}_{t r}^{\beta}(0)\right] \Im H_{\alpha \beta}^{i n}(0)$, which coincides with (3.52). The last component is $\Im H_{n n}^{i n}(0) \dot{\sigma}_{i n}(0)-\Im H_{n \beta}^{i n}(0) \dot{\sigma}_{i n}(0)\left[\dot{q}_{i n}^{\beta}(0)-\dot{q}_{t r}^{\beta}(0)\right]$. Now we add linear 
combination of all upper rows with factor $-\left[\dot{q}_{i n}^{\alpha}(0)-\dot{q}_{t r}^{\alpha}(0)\right]$ to the lowest row. The first $(n-1)$ components of the lowest row coincide now with (3.52), the last component coincides with $(3.54)$, this proves positive definiteness of matrix $\Im H^{\operatorname{tr}}(0)>0$.

We proved the statement and now we can construct solutions to the Riccati equation with required properties

$$
\left\{\begin{array}{l}
H^{r e f}(t)=Z^{r e f}(t)\left(Y^{r e f}(t)\right)^{-1} \\
H^{t r}(t)=Z^{t r}(t)\left(Y^{t r}(t)\right)^{-1}
\end{array}\right.
$$

Now formal Gaussian beam (3.14) is concentrated in the vicinity of the point and propagates along the geodesic $\left(p^{t r}(t) ; q^{t r}(t), \sigma^{t r}(t)\right)\left(\left(p^{r e f}(t) ; q^{r e f}(t), \sigma^{\text {ref }}(t)\right)\right)$ with unit velocity on the manifold.

Next we continue this procedure and obtain higher order terms of the reflected and transmitted phase functions. We omit here these massive technical calculations and obtained formulae, one can find them in [41].

\subsubsection{Phase functions $\Theta_{N}^{r e f}(t, \mathbf{q}, \sigma), \Theta_{N}^{t r}(t, \mathbf{q}, \sigma)$}

We presented the procedure of construction of any finite number of terms in the phase function expansion $\theta_{1}^{\text {ref,tr }}(t), \theta_{2}^{\text {ref,tr }}(t), \ldots, \theta_{N}^{r e f, t r}(t)$. Let us write out Lemma 2.61 conclusions form [38]. Suppose that $\Theta_{N}^{i n}$ is given. Then we can construct $\Theta_{n}^{r e f(t r)}$ such that constructed functions can be presented by series

$$
\Theta^{r e f(t r)}(t, \mathbf{q}, \sigma) \asymp^{N} \Theta_{N}^{r e f(t r)}(t, \mathbf{q}, \sigma)=\sum_{l=1}^{N-1} \theta_{l}^{r e f(t r)}(t)=\sum_{|\iota|=1}^{N-1} \frac{1}{l !} \theta_{l}^{r e f(t r)}(t) \mathbf{Y}^{\iota}(t)
$$

and such that

$$
\Theta_{1}^{r e f(t r)}=\left(P^{r e f(t r)}(t), \mathbf{Y}(t)\right),\left|P^{r e f(t r)}(t)\right|=1
$$

are real $\left(P^{r e f(t r)}(t)\right.$ are determined by (3.28), and $\mathbf{Y}(t)$ are determined by (3.29),

$$
\Theta_{2}^{r e f(t r)}=\frac{1}{2}\left(H^{r e f(t r)}(t) \mathbf{Y}(t), \mathbf{Y}(t)\right), \Im H(t) \geq 0
$$


$H^{t r}(t)$ and $H^{r e f}(t)$ was constructed above. Moreover, the constructed phase functions satisfy conditions (3.6), (3.7) and estimates

$$
\left|\left(\left(\partial_{t}\right)^{2}-g^{i j} \partial_{i} \partial_{j}\right) \Theta_{N}^{r e f(t r)}\right| \leq C_{N}|\mathbf{Y}(t)|^{N},
$$

that means that all requirements form these functions to be Gaussian beam phase functions are satisfied.

\subsection{Reflection and transmission laws}

Consider cotangent space $T_{M_{1} \in_{\gamma}}^{*}\left(\Omega_{-} \cup \Omega_{+}\right)$(see Figure 3.3) and coplane $\pi^{i n}\left(M_{1}\right)$ in it such that the point $M_{1}=(0,0)$ is a point where the beam reaches the interface. The coplane $\pi^{i n}\left(M_{1}\right)$ is a 2 dimensional coplane spanned by covector

$$
P^{i n}(0)=\left.d \Theta^{i n}\right|_{\gamma}\left(M_{1}\right)=\left.\left(\frac{\partial \Theta^{i n}}{\partial q^{1}}, \ldots, \frac{\partial \Theta^{i n}}{\partial \sigma_{-}}\right)\right|_{M_{1}}
$$

and normal covector $d \sigma_{-}=(0, \ldots, 0,-1)$ at the point $M_{1}$ at time $t=0$.

Definition 3.5 (Incidence angle)

We define an incidence angle $\varphi^{\text {in }}$ at the point $M_{1}$ between covectors $P^{\text {in }}(0)=$ $\left.d \Theta^{i n}\right|_{\gamma}(t=0)=\left.\left(\frac{\partial \Theta^{i n}}{\partial q^{1}}, \ldots, \frac{\partial \Theta^{i n}}{\partial \sigma_{-}}\right)\right|_{t=0}$ and $d \sigma_{-}$in the coplane $\pi^{i n}$.

Let $U^{\text {tr }}$ start from $\gamma$ inside $\Omega_{+}$with a transmission angle $\varphi^{t r}$ between the covector $P^{t r}(0)=\left.d \Theta^{t r}\right|_{\gamma}(t=0)=\left.\left(\frac{\partial \Theta^{t r}}{\partial q^{1}}, \ldots, \frac{\partial \Theta^{t r}}{\partial q^{n-1}}, \frac{\partial \Theta^{t r}}{\partial \sigma_{+}}\right)\right|_{t=0}$ and normal covector $d \sigma_{+}=$ $(0, \ldots, 0,1), \pi^{t r} \in T_{M_{1} \in \gamma}^{*}\left(\Omega_{+}\right)$, where the $2 \mathrm{D}$ coplane $\pi^{t r}$ is spanned by covectors $d \sigma_{+}$ and $P^{t r}(0)$. The transmission angle $\varphi^{\text {tr }}$ determine geodesic $\mu^{t r}(t)$.

\section{Definition 3.6 (Reflection angle)}

Similarly we define a reflection angle $\varphi^{\text {ref }}$ at the point $M_{1}$ between covectors $P^{r e f}(0)=\left.d \Theta^{r e f}\right|_{\gamma}(t=0)=\left.\left(\frac{\partial \Theta^{r e f}}{\partial q^{1}}, \ldots, \frac{\partial \Theta^{r e f}}{\partial \sigma_{-}}\right)\right|_{t=0}$ and $d \sigma_{-}$in the coplane $\pi^{r e f}$.

\section{Proposition 3.3.1}

Coplanes $\pi^{i n}, \pi^{r e f}, \pi^{t r}$ coincide in $T_{M_{1} \in \gamma}^{*}\left(\Omega_{-} \cup \Omega_{+}\right)$. If the value $\varphi^{i n}$ of the angle is known then we can find the transmission angle $\varphi^{\text {tr }}$ and the reflection angle $\varphi^{\text {ref }}$. 


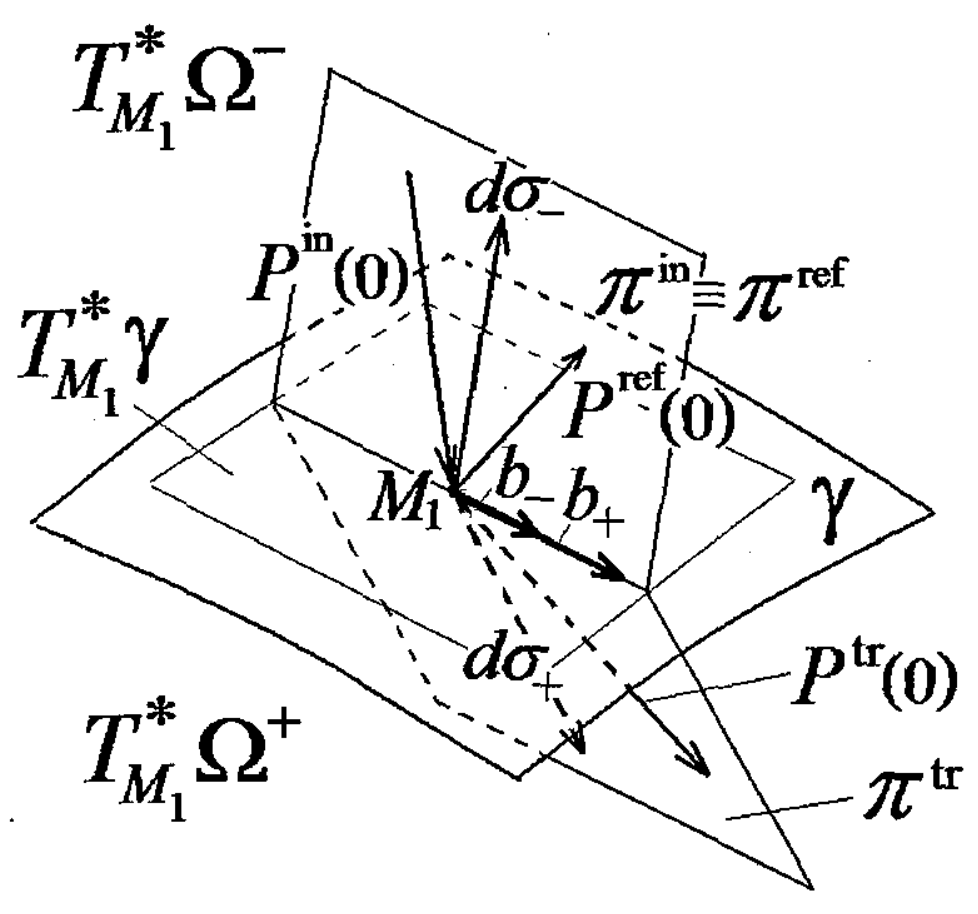

Figure 3.3: Incidence, reflection, transmission coplanes and angles near the interface 
Proof Recalling continuity conditions and differentiating the first one (3.19) with respect to $q^{\alpha}$ (we can not differentiate with respect to $\sigma$ as this condition is valid only on $\gamma$ ) we obtain from equation the coefficients:

$$
\frac{\partial \Theta^{i n}}{\partial q^{\alpha}} l_{\gamma}=\frac{\partial \Theta^{r e f}}{\partial q^{\alpha}} l_{\gamma}=\frac{\partial \Theta^{t r}}{\partial q^{\alpha}} l_{\gamma}
$$

Eikonal equation (3.26) and coordinates normalization at the point $M_{1}$ imply

$$
\frac{\partial \Theta_{N}^{i n}}{\partial \sigma_{-}}\left(M_{1}\right)=\cos \varphi^{i n}=p_{n}^{i n}(0)<0, \frac{\partial \Theta_{N}^{r e f}}{\partial \sigma_{-}}\left(M_{1}\right)=p_{n}^{r e f}(0)=-p_{n}^{i n}(0)>0,
$$

and

$$
\sin \varphi^{i n}=g_{-}^{\alpha \beta} \frac{\partial \Theta^{i n}}{\partial q^{\alpha}} \frac{\partial \Theta^{i n}}{\partial q^{\beta}}\left(M_{1}\right)=g_{-}^{\alpha \beta} p_{\alpha}^{i n}(0) p_{\beta}^{i n}(0) \leq 1
$$

Consider two covectors $P^{i n}(0), d \sigma_{-}$the coplane $\pi^{i n}\left(M_{1}\right)$ is spanned by. Consider two covectors $P^{r e f}(0)$ and $d \sigma_{-}$that the coplane $\pi^{r e f}\left(M_{1}\right)$ is spanned by. We have got the value $P^{r e f}(0)$ above. One can see that all its tangential components coincide with $P^{i n}(0)$. Formula (3.47) implies that they have only the last component $p_{n}^{\text {ref }}$ is different. The covector $P^{r e f}(0)$ can be presented by linear combination $P^{i n}(0)$ and $d \sigma_{-}$, hence they belong to the same $2 \mathrm{D}$ coplane. The coplane $\pi^{r e f}\left(M_{1}\right)$ coincides with the coplane $\pi^{i n}\left(M_{1}\right)$.

Similarly, we consider covectors $P^{i n}(0)$ and $P^{t r}(0)$, they also differ in the last coordinate, as one can see that from (3.48). Compare now $d \sigma_{-}$and $d \sigma_{+}$. Both covectors belong to the same straight line, we present $P^{t r}(0)$ in a form of linear combination of $p^{i n}(0)$ and $d \sigma_{-}$. Thus coplanes $\pi^{i n}\left(M_{1}\right)$ and $\pi^{t r}\left(M_{1}\right)$ coincide.

Introduce two covectors

$$
b_{-} \in \pi^{i n}\left(M_{1}\right) \bigcap T_{M_{1} \in \gamma}^{*} \gamma, b_{+} \in \pi^{t r}\left(M_{1}\right) \bigcap T_{M_{1} \in \gamma}^{*} \gamma
$$

such that

$$
\begin{aligned}
& \left(d \sigma_{-}, b_{-}\right)_{g^{-}}=0,\left|b_{-}\right|_{g^{-}}=\sin \varphi^{i n} \\
& \left(d \sigma_{+}, b_{+}\right)_{g^{+}}=0,\left|b_{+}\right|_{g^{+}}=\sin \varphi^{t r} .
\end{aligned}
$$


Then in equal coplanes $\pi^{r e f}\left(M_{1}\right)$ and $\pi^{i n}\left(M_{1}\right)$ the following is true:

$$
\begin{gathered}
\left(d \Theta^{i n}, d \sigma_{-}\right)=g_{-}^{\alpha \beta}\left(M_{1}\right) \frac{\partial \Theta^{i n}}{\partial q^{\alpha}} b_{\beta}=\sin \varphi^{i n} g_{-}^{\alpha \beta}\left(M_{1}\right) b_{\alpha} b_{\beta}=\sin \varphi^{r e f} g_{-}^{\alpha \beta}\left(M_{1}\right) b_{\alpha} b_{\beta} \\
=\left(d \Theta^{r e f}, d \sigma_{-}\right)=g_{-}^{\alpha \beta}\left(M_{1}\right) \frac{\partial \Theta^{r e f}}{\partial g^{\alpha}} b_{\beta} .
\end{gathered}
$$

Thus $\sin \varphi^{i n}=\sin \varphi^{\text {ref }}$, and eikonal equation gives us a reflected $\operatorname{cosine} \cos \varphi^{\text {ref }}=$ $-\cos \varphi^{\text {in }}$. The latter corresponds to the anisotropic analogue of Frenel's law of geametrical optics, i.e.

$$
\left\{\begin{aligned}
\sin \varphi^{r e f}= & g_{-}^{\alpha \beta} \frac{\partial \Theta^{i n}}{\partial q^{\alpha}} \frac{\partial \Theta^{i n}}{\partial q^{\beta}}\left(M_{1}\right)=g_{-}^{\alpha \beta}\left(M_{1}\right) p_{\alpha}^{i n}(0) p_{\beta}^{i n}(0)=\sin \varphi^{i n}, \\
\cos \varphi^{r e f}= & -\sqrt{1-\left(g_{-}^{\alpha \beta} \frac{\partial \Theta^{i n}}{\partial q^{\alpha}} \frac{\partial \Theta^{i n}}{\partial q^{\beta}}\left(M_{1}\right)\right)}=-\cos \varphi^{i n} . \\
& \left.\frac{\partial \Theta^{i n}}{\partial q^{\alpha}}\right|_{\gamma}=\sin \varphi^{-} \frac{b_{-}}{\mid b_{-} l_{g^{-}}}=\frac{\partial \Theta^{t r}}{\partial q^{\alpha}} l_{\gamma}=\sin \varphi^{+} \frac{b_{+}}{\mid b_{+} l_{g^{+}}}
\end{aligned}\right.
$$

The latter implies the formula for the cosine of the transmitted wave $\cos \varphi^{t r}$ expressed in terms of given function of the incident wave, For the Gaussian beam $U^{\text {tr }}$ to propagate inside $\Omega_{+}$we should take the positive sign in the square root

$$
\left\{\begin{array}{l}
\cos \varphi^{t r}=+\sqrt{1-\sin ^{2} \varphi^{i n} \frac{g_{+}^{\alpha \beta} b_{\alpha} b_{\beta}}{g_{-}^{\alpha \beta} b_{\alpha} b_{\beta}}}>0 \\
\sin \varphi^{t r}=\sin \varphi^{i n} \sqrt{\frac{g_{+}^{\alpha \beta} b_{\alpha} b_{\beta}}{g_{-}^{\alpha \beta} b_{\alpha} b_{\beta}}}
\end{array}\right.
$$

We found the values of the reflected and transmitted angles, determined by their cosines (3.63) and (3.64) in terms of the incident field.

Formula (3.64) rewritten in the form of sinuses ratio corresponds to the Snell's law. One can see that in the case of $\varphi^{t r}=\frac{\pi}{2}$, the angle $\varphi^{\text {in }}$ becomes critical, the transmitted wave propagates in a tangential direction to the interface. In this case the ray expansions could not be used, but such a kind of propagation is beyond the scope of this work. 


\subsection{Amplitudes}

\subsubsection{Amplitude values on the interface}

We rewrite interface conditions such that we are able to express boundary data of the reflected and transmitted waves in terms of incident field, as we did it for the phase function.

Lemma 3.3 Let $u_{N}^{i n}(t, q, 0)$ be known trace of the incident amplitude function on the interface. We can find traces of the reflected and transmitted amplitudes $u_{N}^{r e f}(t, q, 0)$ and $u_{N}^{t r}(t, q, 0)$ on the interface for any $N>0$.

Proof We will prove this by giving the construction procedure.

Consider continuity conditions (3.19) on the interface and substitute the formal Gaussian beam series into it. The condition of continuity of the Gaussian beam gives us the following equations

$$
\left\{\begin{array}{l}
\left.\left.\left.\Theta^{i n}(\mathrm{x}, t)\right|_{\gamma} \asymp \Theta^{r e f}(\mathrm{x}, t)\right|_{\gamma} \asymp \Theta^{t r}(\mathrm{x}, t)\right|_{\gamma}, \\
\left.u_{k}^{i n}(\mathrm{x}, t)\right|_{\gamma}+\left.u_{k}^{r e f}(\mathrm{x}, t)\right|_{\gamma}=\left.u_{k}^{t r}(\mathrm{x}, t)\right|_{\gamma}, \quad \forall k .
\end{array}\right.
$$

The continuity condition for normal derivatives of the Gaussian beam implies equations

$$
\begin{array}{r}
\frac{1}{(i \varepsilon)} \sum_{l=0}^{N}(i \varepsilon)^{l}\left(S^{i n} u_{l}^{i n}+S^{r e f} u_{l}^{r e f}\right) l_{\gamma}+\sqrt{g_{-}} \sum_{l=0}^{N}(i \varepsilon)^{l}\left(\frac{\partial u_{l}^{i n}}{\partial \sigma_{-}}+\frac{\partial u_{l}^{r e f}}{\partial \sigma_{-}}\right) l_{\gamma} \\
=\frac{1}{(i \varepsilon)} \sum_{l=0}^{N}(i \varepsilon)^{l}\left(S^{t r} u_{l}^{t r}\right) l_{\gamma}+\sqrt{g_{+}} \sum_{l=0}^{N}(i \varepsilon)^{l}\left(\frac{\partial u_{l}^{t r}}{\partial \sigma_{+}}\right) l_{\gamma}
\end{array}
$$

where we used some useful notations:

$$
S^{\text {in,ref }}:=\left(\sqrt{g_{-}(q, 0)} \frac{\partial \Theta_{N}^{\text {in,ref }}}{\partial \sigma_{-}}(t, q, 0)\right), S^{t r}:=\left(\sqrt{g_{+}(q, 0)} \frac{\partial \Theta_{N}^{t r}}{\partial \sigma_{+}}(t, q, 0)\right) .
$$


Equating coefficients of similar orders of $(i \varepsilon)$, assuming that

$$
u_{0}^{i n, r e f, t r} \not \equiv 0, \quad u_{k}^{i n, r e f, t r} \equiv 0, \forall k<0,
$$

we get the system of equations for $k=-1,0,1, \ldots$ :

$$
\mathbf{P}\left(\begin{array}{c}
u_{k+1}^{t r}(t, q, 0) \\
u_{k+1}^{r e f}(t, q, 0)
\end{array}\right)=\mathbf{Q} u_{k+1}^{i n}(t, q, 0)+\mathbf{R}_{k}
$$

where

$$
\begin{gathered}
\mathbf{P}:=\left(\begin{array}{cc}
S^{t r} & -S^{r e f} \\
-1 & 1
\end{array}\right), \mathbf{Q}:=\left(\begin{array}{c}
S^{\text {in }} \\
-1
\end{array}\right), \mathbf{R}_{k}:=\left(\begin{array}{c}
\mathcal{R}\left(u_{k}\right) \\
-1
\end{array}\right), \\
\mathcal{R}\left(u_{k}\right):=-\sqrt{g_{-}(q, 0)}\left(\frac{\partial u_{k}^{i n}}{\partial \sigma}(t, q, 0)+\frac{\partial u_{k}^{r e f}}{\partial \sigma}(t, q, 0)\right)+\sqrt{g_{+}(q, 0)} \frac{\partial u_{k}^{t r}}{\partial \sigma}(t, q, 0) .
\end{gathered}
$$

We denote the determinant of the obtained system by

$$
\mathcal{D}:=\left(-S^{t r}+S^{r e f}\right) \neq 0 \text {. }
$$

The obtained system for the polynomials on $\gamma(3.67)$ has a unique solution for any RHS, as the determinant (3.69) is $\mathcal{D}_{k} \neq 0$ for any $k>0$. We tackle the system of equations and obtain

$$
\left(\begin{array}{c}
u_{k+1}^{t r}(t, q, 0) \\
u_{k+1}^{r e f}(t, q, 0)
\end{array}\right)=\mathbf{P}^{-1} \mathbf{Q} u_{k+1}^{i n}(t, q, 0)+\mathbf{P}^{-1} \mathbf{R}_{k}
$$

where

$$
\mathbf{P}^{-1}:=\frac{1}{\mathcal{D}}\left(\begin{array}{cc}
1 & S^{r e f} \\
1 & S^{t r}
\end{array}\right)
$$

Thus we have found the recurrent formulae for any finite number of boundary values on $\gamma$ for the reflected and transmitted amplitudes in terms of known incident field. Hence Lemma 3.3 is proved. $\square$

Remark 3.4.1 (First amplitude terms) We present the main terms $u_{0}^{r e f}(t, q, 0)$ and $u_{0}^{t r}(t, q, 0)$ as following:

$$
\left(\begin{array}{c}
u_{0}^{\text {tr }}(t, q, 0) \\
u_{0}^{r e f}(t, q, 0)
\end{array}\right)=\mathbf{P}^{-1} \mathbf{Q} u_{0}^{i n}(t, q, 0)=\left(\begin{array}{c}
\mathcal{T} \\
\mathcal{R}
\end{array}\right) u_{0}^{i n}(t, q, 0),
$$


Notation 3.3 (Reflection and Transmission Coefficients) Coefficients $\mathcal{R}$ and $\mathcal{T}$ in (3.72) are reflection and transmission coefficients correspondingly, such that $1+\mathcal{R}=\mathcal{T}$.

Similarly,

$$
\left(\begin{array}{c}
u_{1}^{t r}(t, q, 0) \\
u_{1}^{r e f}(t, q, 0)
\end{array}\right)=\mathbf{P}^{-1} \mathbf{Q} u_{1}^{i n}(t, q, 0)+\mathbf{P}^{-1} \mathbf{R}_{0}=\left(\begin{array}{c}
\mathcal{T} \\
\mathcal{R}
\end{array}\right) u_{1}^{i n}(t, q, 0)+\mathbf{P}^{-1} \mathbf{R}_{0}
$$

\subsubsection{Amplitude equations}

We write out ODE for the amplitude functions $u^{r e f}(t, q, \sigma)$ and $u^{t r}(t, q, \sigma)$. To solve them uniquely we have to supply them with initial data. These initial data will be obtained later. The investigation of transport equations (3.25) on geodesic $\mu(t)$ is based on Tailor's expansion. For any $l$ the expansion is

$$
u_{l}(t, \mathbf{q}, \sigma) \asymp \sum_{m \geq 0} u_{l(m)}(t, q-q(t), \sigma-\sigma(t)) \asymp \sum_{m \geq 0} \hat{u}_{l(m)}(\hat{t}, \hat{\xi}),
$$

here $u_{l(m)}, \hat{u}_{l(m)}$ are homogeneous polynomials of order $m, m=0,1, \ldots$ with respect to $t, q, \sigma$ and $\hat{t}, \hat{\xi}$ are $\hat{t}=t, \hat{y}=Y^{-1}(t) \mathbf{Y}(t)$. Operator $\mathcal{L}_{\Theta_{N}}$ is the linear differential operator of the first order, it depends only on such coefficients $\hat{u}_{l(m)}$ that $m \leq l+1$ in equation (3.25). Those terms that are contained in $u_{l(m+1)}$ are equal to zero along the bi-characteristics because of Hamilton system (3.34). Similarly, $\hat{u}_{l(m)}$ as a function of time $t$ has values in the space of homogeneous polynomials of order $m$ with respect to $\hat{\xi}$. Then ODE for $\hat{u}_{l(m)}$ has a form

$$
\frac{d}{d t} \hat{u}_{l(m)}(t)+r(t) \hat{u}_{l(m)}(t)=\hat{\mathcal{F}}_{l(m)}(t), l=0,1, \ldots,
$$

$\hat{\mathcal{F}}_{l(m)}(t)$ are homogeneous polynomials of order $m$ that depend on $\hat{u}_{s(p)}(t)$ and $\hat{\theta}_{N, p}$ only as $p \leq m+2, s<l$. As

$$
r(t)=-\frac{1}{2} \operatorname{tr}\left(B^{t}+C H\right)+\frac{1}{4} \frac{d}{d t} \ln g(t), H(t)=Z(t) Y^{-1}(t),
$$


i.e.

$$
r(t)=\frac{1}{2} \frac{d}{d t} \ln [\operatorname{det} Y(t)]+\frac{1}{4} \frac{d}{d t} \ln g(t),
$$

then we can solve these equations once supplied them with initial data:

$$
\begin{gathered}
\hat{u}_{m, l}(\hat{t})=\varrho(t)\left(\hat{u}_{m, l}(0)+\int_{0}^{t} \varrho^{-1}\left(t^{\prime}\right) \hat{\mathcal{F}}_{m, l}\left(t^{\prime}\right) d t^{\prime},\right) \\
\varrho(t)=\sqrt{\frac{\operatorname{det} Y(0)}{\operatorname{det} Y(t)}} \sqrt[4]{\frac{g(0)}{g(t)}} .
\end{gathered}
$$

Note that $\operatorname{det} Y^{-1}(t)$ corresponds to the geometric divergence of ray field. In this section we used well-known procedure from, say, [6], see also [38].

\subsubsection{Initial values}

Consider now obtained above amplitude representations (we omit captions ref,tr, as the following is valid for both amplitudes):

$$
\left\{\begin{array}{l}
u(t, q, \sigma) \asymp^{N} \sum_{l=0}^{N} u_{l}(i \varepsilon)^{l}, \text { see }(3.11), \\
u_{l}(t, q, \sigma)=u_{l(0)}+\ldots+u_{l(L(N))}, L(N)=\frac{2(N+1-l)}{1-2 \eta}, \\
u_{l}^{r e f, t r}(t, q, 0) \text { are found, see }(3.70), \\
u_{l}^{r e f, t r}(0, q, \sigma) \text { are the goals of this subsection 3.4.3. }
\end{array}\right.
$$

Again as before we will use Tailor's expansions. Rewrite (3.11) similarly to the way we rewrote the phase expansions (3.27). Simultaneously we introduce some special notations for the homogeneous polynomials in series (3.11) in order to reduce the number of indices. We construct just several first terms, the rest terms can be obtained similarly. We omit here all calculations, one can find them in [41]. The initial data for the reflected and transmitted amplitude functions can be obtained in terms of the given incident field. For instance, one can show that

$$
\left\{\begin{array}{l}
u_{0(0)}^{r e f}(t)=\varrho(t) \mathcal{R} u_{0(0)}^{i n}(0), \\
u_{0(0)}^{t r}(t)=\varrho(t) \mathcal{T} u_{0(0)}^{i n}(0) .
\end{array}\right.
$$




\subsection{Exact and Approximate Solutions Estimates}

We have constructed all terms of finite Gaussian beam. Now we will show that for any $N$ there exists an exact solution - Gaussian beam, corresponding to this finite Gaussian beam of order $N$. So, we have constructed formal Gaussian beam $U(\varepsilon ; t, q, \sigma)$, formal Gaussian beam $U_{N}(\varepsilon ; t, q, \sigma)$, corresponding to $U(\varepsilon ; t, q, \sigma)$ and the required exact solution $\mathcal{U}_{N}(t, q, \sigma)$ to the following problem

$$
\left\{\begin{array}{l}
\square_{ \pm} \mathcal{U}_{N}=0 \text { in }\left(\Omega_{-} \cup \Omega_{+}\right) \times\left[-t_{0}, t_{0}\right], \\
\left.\mathcal{U}_{N}^{-}\right|_{\gamma}=\left.\mathcal{U}_{N}^{+}\right|_{\gamma} \\
\left.\sqrt{g^{-}} \frac{\partial}{\partial \sigma_{-}} \mathcal{U}_{N}^{-}\right|_{\gamma}=\left.\sqrt{g^{+}} \frac{\partial}{\partial \sigma_{+}} \mathcal{U}_{N}^{+}\right|_{\gamma} .
\end{array}\right.
$$

As we know only the approximation $U_{N}$ to the required solution $\mathcal{U}_{N}$, we consider an approximation $\chi U_{N}$ (here $\chi$ is a smooth cut-off function in $t$ and all spacial variables) to the solution of the problem (3.79):

$$
\left\{\begin{array}{l}
\square_{g_{-}} \chi\left(U_{N}^{i n}+U_{N}^{r e f}\right)=R_{0}^{-} \quad \text { in } \Omega_{-}, \\
\square_{g_{+}}\left(\chi U_{N}^{t r}\right)=R_{0}^{+} \text {in } \Omega_{+}, \\
\left.\chi\left(U_{N}^{i n}+U_{N}^{r e f}\right)\right|_{\gamma}=\left.\chi U_{N}^{t r}\right|_{\gamma}+R_{1} \\
\left.\sqrt{g^{-}} \frac{\partial}{\partial \sigma_{-}} \chi\left(U_{N}^{i n}+U_{N}^{r e f}\right)\right|_{\gamma}=\left.\sqrt{g^{+}} \frac{\partial}{\partial \sigma_{+}} \chi U_{N}^{t r}\right|_{\gamma}+R_{2}
\end{array}\right.
$$

One can show that

$$
R_{0}^{-}<C_{0}^{-}\left(2 t_{0}\right) \varepsilon^{N} M_{\varepsilon}, R_{0}^{+}<C_{0}^{+}\left(t_{0}\right) \varepsilon^{N} M_{\varepsilon}, R_{1}<C_{1} \varepsilon^{N+1} M_{\varepsilon}, R_{2}<C_{2} \varepsilon^{N} M_{\varepsilon}
$$

Notation 3.4 (Polynomially small function) We say that function $\psi$ is polynomially small of order $k$, if $\|\psi\|_{C^{k}} \leq \varepsilon^{k}$.

The last formula implies now that $R_{0}^{-}, R_{0}^{+}$are polynomially small of order $N-\frac{n}{4}$, $R_{1}, R_{2}$ are polynomially small of order $N+1-\frac{n}{4}$. The inhomogeneous interface 
conditions on $\gamma$ are to be replaced by the homogeneous ones by introducing a new function $F$ in $\Omega_{-} \bigcup \Omega_{+}$. Thus we get a new problem for $F$

$$
\left\{\begin{array}{l}
\square_{g^{+}} F=s \text { in } \Omega_{+}, \\
\left.F\right|_{\gamma}=R_{1}, \\
\left.\sqrt{g^{+}} \frac{\partial}{\partial \sigma_{+}} F\right|_{\gamma}=R_{2} .
\end{array}\right.
$$

Note that $F$ is polynomially small of order $N+1-\frac{n}{4}$ in $\Omega_{-} \cup \Omega_{+}$. The RHS of (3.81), or $s$, is also polynomially small of order $N+1$. We can choose function $F$ to be the following

$$
F=R_{1}(t, q) \tilde{\chi}(\sigma)+\left.\frac{R_{2}}{\sqrt{g_{+}}}\right|_{\sigma=0} \tilde{\chi}(\sigma) \sigma .
$$

where $\tilde{\chi}$ is a cut-off function. Next we introduce new function

$$
W_{N}=\left\{\begin{array}{l}
W_{N}^{-}=\chi\left(U_{N}^{i n}+U_{N}^{r e f}\right) \text { in } \Omega_{-}, \\
W_{N}^{+}=F+\chi U_{N}^{t r} \text { in } \Omega_{+} .
\end{array}\right.
$$

Thus we get a problem for $W_{N}$ and the corresponding interface continuity conditions on $\gamma$ are satisfied:

$$
\left\{\begin{array}{l}
\square_{ \pm} W_{N}=\mathcal{E}=\left\{\begin{array}{l}
\mathcal{E}^{-}, \text {in } \Omega_{-} \\
\mathcal{E}^{+}, \text {in } \Omega_{+}
\end{array},\right. \\
\left.W_{N}^{-}\right|_{\gamma}=\left.W_{N}^{+}\right|_{\gamma}, \\
\left.\sqrt{g^{-}} \frac{\partial}{\partial \sigma} W_{N}^{-}\right|_{\gamma}=\left.\sqrt{g^{+}} \frac{\partial}{\partial \sigma} W_{N}^{+}\right|_{\gamma}
\end{array}\right.
$$

Here $\mathcal{E}^{-}<C_{0}^{-}\left(2 t_{0}\right) \mathcal{E}^{\left(N-\frac{n}{4}\right)}, \mathcal{E}^{+}<C_{0}^{+}\left(t_{0}\right) \mathcal{E}^{\left(N-\frac{n}{4}\right)}$, i.e. $\mathcal{E}^{-}$and $\mathcal{E}^{+}$are polynomially small of order $N-\frac{n}{4}$. The solution $W_{N}$ to the problem (3.82) satisfies exactly the interface continuity conditions (3.18). Recall that $\mathcal{E} \in C^{\alpha(N)}\left(t ; L^{2}\left(\Omega_{-} \bigcup \Omega_{+}\right)\right)$, i.e. there is a big number of time derivatives of $\mathcal{E}$ with values from $L^{2}$ in the whole region. Compare now $W_{N}$ with the exact formally written solution $\mathcal{U}_{N}$, as $\square \mathcal{U}_{N}$ is determined 
in a sense of the Dirichlet form existence (it contains the interface conditions)

$$
\left\{\begin{array} { l } 
{ \square _ { \pm } \mathcal { U } _ { N } = 0 , } \\
{ \mathcal { U } _ { N } ^ { - } | _ { \gamma } = \mathcal { U } _ { N } ^ { + } | _ { \gamma } } \\
{ \sqrt { g _ { - } } \frac { \partial } { \partial \sigma _ { - } } \mathcal { U } _ { N } ^ { - } | _ { \gamma } = \sqrt { g _ { + } } \frac { \partial } { \partial \sigma _ { + } } \mathcal { U } _ { N } ^ { + } | _ { \gamma } } \\
{ \mathcal { U } _ { N } | _ { - t _ { 0 } } = U _ { N } ^ { i n } | _ { - t _ { 0 } } , } \\
{ \partial _ { t } \mathcal { U } _ { N } | _ { - t _ { 0 } } = \partial _ { t } U _ { N } ^ { i n } | _ { - t _ { 0 } } , }
\end{array} \quad \left\{\begin{array}{l}
\square_{ \pm} W_{N}=\mathcal{E} \\
\left.W_{N}^{-}\right|_{\gamma}=\left.W_{N}^{+}\right|_{\gamma} \\
\left.\sqrt{g_{-}} \frac{\partial}{\partial \sigma_{-}} W_{N}^{-}\right|_{\gamma}=\left.\sqrt{g_{+}} \frac{\partial}{\partial \sigma_{+}} W_{N}^{+}\right|_{\gamma} \\
\left.W_{N}\right|_{-t_{0}}=\left.\chi U_{N}^{i n}\right|_{-t_{0}} \\
\left.\partial_{t} W_{N}\right|_{-t_{0}}=\left.\partial_{t}\left(\chi U_{N}^{i n}\right)\right|_{-t_{0}}
\end{array}\right.\right.
$$

It is clear that $\square_{ \pm}\left(\mathcal{U}_{N}-W_{N}\right)=-\mathcal{E}$. Let us a give a notation to the difference of solutions, say $-\mathcal{E}=\square_{ \pm} V$. The initial data coincide (in the domain of the LaplaceBeltrami operator $\left.\mathcal{D}\left(\left(\Delta_{g}\right)^{q}\right), q>2\right)$. Our problem is linear thus we consider solution:

$$
V_{N}=\mathcal{U}_{N}-W_{N}:\left\{\begin{array}{l}
\square_{ \pm} V_{N}=\square_{ \pm}\left(\mathcal{U}_{N}-W_{N}\right)=-\mathcal{E}, \\
\left.V_{N}\right|_{-t_{0}}=\left.\partial_{t} V_{N}\right|_{-t_{0}}=0, \\
\left.V_{N}\right|_{\left(\partial \Omega_{-} \cup \partial \Omega_{+}\right) \times\left[-t_{0}, t_{0}\right]}=0 .
\end{array} .\right.
$$

Here we assumed that at time $t_{0}>0$ the beam has not yet reached the boundary $\partial \Omega_{-} \cup \partial \Omega_{+}$

\subsubsection{Convergence}

This follows from the construction procedure that the RHS $\mathcal{E}$ of (3.83) is small on the time interval $\left[-t_{0}, t_{0}\right]$ with values from $L^{2}$. All time derivatives (there is a large number) are also from $L^{2}$ and are small.

The main idea is to estimate the difference $V_{N}$ between the exact solution and the constructed finite Gaussian beam corresponding to it. We write out Fourier series of $V_{N}$ and $\mathcal{E}$ with respect to their eigenfunctions $\Delta_{g} \varphi_{k}=\lambda_{k} \varphi_{k}$ :

$$
V_{N}(t, \mathbf{x})=\sum_{k=1}^{\infty} v_{k}(t) \varphi_{k}(\mathbf{x}), \quad v_{k}\left(-t_{0}\right)=\dot{v}_{k}\left(-t_{0}\right)=0
$$




$$
\mathcal{E}(t, \mathbf{x})=\sum e_{k}(t) \varphi_{k}(\mathbf{x})
$$

Then

$$
\square V_{N}(t, \mathbf{x})=\sum\left[\ddot{v}_{k}(t)+\lambda_{k} v_{k}(t)\right] \varphi_{k}(\mathbf{x})=\sum e_{k}(t) \varphi_{k}(\mathbf{x})
$$

where as $e_{k}(t)$ are small and all its time derivatives are polynomially small, we has $\sum\left|\frac{\partial^{p}}{\partial t^{p}} e_{k}(t)\right|^{2}<C_{p}\left(t_{0}\right) \varepsilon^{L}<\infty$, where $p \leq L=\left(N-p-\frac{n}{4}\right)^{2}$, then

$$
\ddot{v}_{k}(t)+\lambda_{k} v_{k}(t)=e_{k}(t)
$$

and then

$$
v_{k}(t)=\int_{-\infty}^{t} \frac{\sin \sqrt{\lambda_{k}}\left(t-t^{\prime}\right)}{\sqrt{\lambda_{k}}} e_{k}\left(t^{\prime}\right) d t^{\prime}
$$

We integrate the latter by parts, hence

$$
I_{1}:=\left.\frac{-1}{\lambda_{k}} \cos \sqrt{\lambda_{k}}\left(t-t^{\prime}\right) e_{k}\left(t^{\prime}\right)\right|_{\infty} ^{t}+\int_{-\infty}^{t} \frac{1}{\lambda_{k}} \cos \sqrt{\lambda_{k}}\left(t-t^{\prime}\right) e_{k}^{\prime}\left(t^{\prime}\right) d t^{\prime},
$$

where $e_{k}(-\infty)=0$, then

$$
I_{1}=\left.\frac{1}{\lambda_{k}} e_{k}(t)\right|_{-\infty} ^{t}+\frac{1}{\lambda_{k}} \int_{-\infty}^{t} \cos \sqrt{\lambda_{k}}\left(t-t^{\prime}\right) e_{k}^{\prime}\left(t^{\prime}\right) d t^{\prime} .
$$

Consider firstly the second summand, i.e. the integral by introducing new notation for the integrand:

$$
I_{2}:=\frac{1}{\lambda_{k}} \int_{-\infty}^{t} \cos \sqrt{\lambda_{k}}\left(t-t^{\prime}\right) e_{k}^{\prime}\left(t^{\prime}\right) d t^{\prime}=\frac{1}{\lambda_{k}} \int_{-\infty}^{t} r\left(t, t^{\prime}\right) d t^{\prime} .
$$

We estimate a new function

$$
\sum r_{k}(t) \varphi_{k}(\mathbf{x})=r(t)
$$

using Parseval inequality: $\left\|r_{k}(t)\right\|^{2}=\sum\left|r_{k}(t)\right|^{2}$, and then the Cauchy-Shwartz inequality we get

$$
\left|r_{k}(t)\right|^{2} \leq \int_{-t_{0}}^{t} \cos ^{2} \sqrt{\lambda_{k}}\left(t-t^{\prime}\right) \int_{-t_{0}}^{t}\left|e_{k}^{\prime}\left(t^{\prime}\right)\right|^{2} d t^{\prime}<\left(t-t_{0}\right) \int_{-t_{0}}^{t}\left|e_{k}^{\prime}\left(t^{\prime}\right)\right|^{2} d t^{\prime} .
$$


The latter implies

$$
I_{3}:=\left\|r_{k}(t)^{2}\right\| \leq\left(t+t_{0}\right) \int_{-t_{0}}^{t} \sum\left|e_{k}^{\prime}\left(t^{\prime}\right)\right|^{2} d t^{\prime} .
$$

As $\sum\left|e_{k}^{\prime}\left(t^{\prime}\right)\right|^{2}$ is positive thus we change sign, the result is an $L^{2}$-norm on $\mathcal{E}$, we have

$$
I_{3}=\left(t+t_{0}\right) \int_{-t_{0}}^{t}\left\|\mathcal{E}\left(t^{\prime}\right)\right\|^{\prime} d t^{\prime} \leq 2 t_{0} \int_{-t_{0}}^{t_{0}}\left\|\mathcal{E}\left(t^{\prime}\right)\right\|_{L^{2}}^{2} d t^{\prime}:=E
$$

where $\left\|\mathcal{E}\left(t^{\prime}\right)\right\|_{L^{2}}$ is polynomially small of order $\left(N-\frac{n}{4}\right)$. Let us estimate the first summand, as $\frac{-1}{\lambda_{k}} e_{k}(t)$ is from $H^{1} \cap \mathcal{D}(\Delta)$, then for any $t$

$$
\sum \lambda_{k}^{2}\left|v_{k}(t)\right|^{2}=\sum\left|e_{k}\right|^{2}+E<\infty
$$

We showed that $v_{k}(t)$ is small in the $H^{1}$-norm. Consider $\dot{v}_{k}(t)$ :

$$
\begin{gathered}
\dot{v}_{k}=\int_{-\infty}^{t} \cos \sqrt{\lambda_{k}}\left(t-t^{\prime}\right) e_{k}\left(t^{\prime}\right) d t^{\prime}=-\frac{1}{\lambda_{k}} \int_{-\infty}^{t} \sin \sqrt{\lambda_{k}}\left(t-t^{\prime}\right) e_{k}^{\prime}\left(t^{\prime}\right) d t^{\prime}, \\
\sum\left|\dot{v}_{k}(t)\right|^{2} \asymp \widetilde{C}(t) \int \frac{\left|e_{k}^{\prime}\left(t^{\prime}\right)\right|^{2} d t^{\prime}}{\lambda_{k}}<\infty .
\end{gathered}
$$

Inequality (3.87) says that $\Delta V_{N} \in L^{2}$, and that $V_{N} \in \mathcal{D}(\Delta)$ correspondingly. Function $\mathcal{E}$ can be time differentiated; similarly to inequality (3.89) one can estimate time derivatives of $V_{N}$. In particular, (3.89) implies that $V_{t}^{\prime} \in L^{2}$. The result can be rewritten as:

$$
V_{N} \in C^{p(N)}\left(\left[-t_{0}, t_{0}\right], \mathcal{D}(\Delta)\right), p \geq 1
$$

We proved the statement (3) of Theorem 3.1. 


\subsection{Conclusion}

We constructed phase and amplitude functions of Gaussian beams of "quasiphoton" type for the incident, reflected and transmitted wave fields near the interface $\gamma$ in sections (3.2), (3.4).

We assumed the incident field to be given $\left(f^{0}, V^{0}, \Gamma_{0}, \Theta^{0}, M_{0},-t_{0}\right)$, ) see (3.2), (3.4). We used its trace on the interface, continuity conditions and Hamilton-Jacobi equations for construction of the reflected and transmitted fields as a formal expansions, checking that all homogeneous polynomials in these expansion satisfy condition for these series to be formal Gaussian beam. We truncated these formal series on order $N$ and showed that for any $N$ there exists an exact solution to the wave equation, which is asymptotically close to constructed finite Gaussian beam.

One can write out the analogue of theorem 3.19 (form [38])'s result for "quasiphotons" propagating from the interface along geodesics which are not normal to $\gamma$. The corresponding geodesics $\mu^{r e f, t r}(t)$ are such that the directing cosine at $M_{0}$ is

$$
\left.\cos \varphi^{r e f, t r}\right|_{t=0}=\dot{\sigma}_{r e f, t r}(0) \geq 0
$$

Phase function $\Theta_{N}^{r e f, t r}$ and amplitude function $\sum_{l=1}^{N} u_{l}^{r e f, t r}$ satisfy (3.65) on $\gamma$. Each term of these functions as an homogeneous polynomial is a solution to the ODE (3.22), (3.25) with respect to time taking into account constructed initial data.

In more details these results can be found in [41]. 


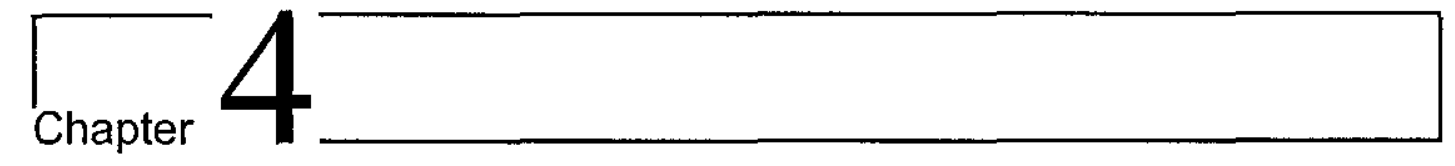

\section{IBSP for a Smooth Riemannian Manifold}

\subsection{Reconstruction from BSD given on the boundary}

This section is a brief description of the boundary control method taken from [38]. Here we consider $\mathcal{M}$ to be a smooth Riemannian manifold of dimension $n$ with smooth boundary $\partial \mathcal{M}$.

\subsubsection{Formulation of the smooth problem}

We consider Laplace-Beltrami operator $\Delta$ in $L^{2}(\mathcal{M}, d V)$ on $\mathcal{M}$ with boundary Dirichlet boundary condition.

$$
\begin{array}{r}
\Delta u=\Delta_{g} u=-g^{-1 / 2} \partial_{j}\left(g^{1 / 2} g^{j k} \partial_{k} u\right) \\
\mathcal{D}(\mathcal{A})=H^{2}(\mathcal{M}) \cap H_{0}^{1}(\mathcal{M}), \\
d V=V_{g}=g^{1 / 2} d x^{1} \wedge \ldots \wedge d x^{n},
\end{array}
$$

here $H^{2}, H_{0}^{1}$ are Sobolev spaces, $g$ is a metric tensor of the manifold $\mathcal{M}$ is a real-valued smooth function on $\mathcal{M}$. Denoting the eigenvalues and the orthonormal eigenfunctions of $\Delta$ by $\lambda_{j}$ and $\varphi_{j}, j=1,2, \ldots$, correspondingly, we have the following definition. 


\section{Definition 4.1 (Boundary spectral data (BSD))}

The collection

$$
\left\{\Gamma \subset \partial \mathcal{M}, \lambda_{j},\left.\partial_{\nu} \varphi_{j}\right|_{\partial \mathcal{M}}, j=1,2 \ldots\right\}
$$

is the set of boundary spectral data of $(\mathcal{M}, \Delta), B S D(\Delta, \Gamma)$ where $\left.\partial_{\nu} \varphi\right|_{\Gamma}=\left.\nu^{j} \partial_{j} \varphi\right|_{\Gamma}$ are the traces of normal derivatives of eigenfunctions, $\quad \nu=\left\{\nu^{j}\right\}$ is a unit inward normal to $\partial \mathcal{M}$.

Problem 4.1 Let $\left\{\partial \mathcal{M}, \lambda_{j},\left.\partial_{\nu} \varphi_{j}\right|_{\partial \mathcal{M}}, j=1,2 \ldots\right\}$ be the given $\operatorname{BSD}(\Delta, \partial \mathcal{M})$ of a Laplace-Beltrami operator $-\Delta_{g}$. Do this data determine uniquely the Riemannian manifold $(\mathcal{M}, g)$ ?

Statement 4.1.1.1 Assume $(\mathcal{M}, \Delta)$ and $(\widetilde{\mathcal{M}}, \widetilde{\Delta})$ are 2 pairs of smooth compact Riemannian manifolds and Laplace-Beltrami operators. Assume there are open sets $\Gamma \in$ $\partial \mathcal{M}$ and $\tilde{\Gamma} \in \widetilde{\partial \mathcal{M}}: B \boldsymbol{B D}(\Gamma)=\boldsymbol{B S D}(\widetilde{\Gamma})$ namely $\Gamma=\widetilde{\Gamma} ; \lambda_{k}=\widetilde{\lambda}_{k},\left.\partial_{\nu} \varphi_{k}\right|_{\Gamma}=\left.\partial_{\nu} \widetilde{\varphi}_{k}\right|_{\tilde{\Gamma}}$, then operators $\Delta$ and $\widetilde{\Delta}$ are equal, manifolds $\mathcal{M}$ and $\widetilde{\mathcal{M}}$ are isometric.

This statement is proved in [38], the brief description of the proof scheme is given in the following sections.

\subsubsection{Reconstruction of the Fourier coefficients of the waves}

We consider the following problem (see [46], [1], [32] [38], [27])

$$
\begin{cases}\square_{g} u=\partial_{t}^{2} u-\Delta_{g} u=0 & \text { in } \quad Q^{T}=\mathcal{M} \times[0, T], \\ \left.u\right|_{\Sigma^{T}}=f(t, x), & \Sigma^{T}=\partial \mathcal{M} \times[0, T], \\ \left.u\right|_{t=0}=\left.\partial_{t} u\right|_{t=0}=0, & \end{cases}
$$

so we can find the smoothness classes for the solution of (4.5) as follows depending on the smoothness of the boundary source $f$ : 
- $u \in C\left([0, T] ; H^{1}(\mathcal{M})\right) \cap C^{1}\left([0, T] ; L^{2}(\mathcal{M})\right)$ when $f \in H^{1}\left(\Sigma^{T}\right) \quad$ and $\left.\quad f\right|_{t=0}=0$

- $u \in C\left([0, T] ; L^{2}(\mathcal{M})\right)$ when $f \in L^{2}\left(\Sigma^{T}\right)$.

Notation 4.1 (Wave produced by boundary source) We denote by $u^{f}(t)$ the solution to problem (4.5) with boundary source function $f(t)$.

We can represent our function as a sum of its Fourier coefficients $u_{k}^{f}(t)$ over our "eigenfunctional" basis $\varphi_{k}$ :

$$
u^{f}(t)=\sum_{k=1}^{\infty} u_{k}^{f}(t) \varphi_{k}
$$

The Fourier coefficients $u_{k}^{f}(t)$ are smooth functions:

- $u_{k}^{f}(t) \in C^{1}([0, T])$ if $f \in H^{1}\left(\Sigma^{T}\right)$ and $\left.f\right|_{t=0}=0$,

- $u_{k}^{f}(t) \in C([0, T])$ if $f \in L^{2}\left(\Sigma^{T}\right)$.

Assuming that $f \in C^{\infty}\left(\Sigma^{T}\right)$ and $\left.\partial_{t}^{p} f\right|_{t=0}=0$, for all $p=0,1, \ldots$, and differentiating $u_{k}^{f}(t)$ twice over $t$ we have:

$$
\frac{d^{2}}{d t^{2}} u_{k}^{f}(t)=\int_{\mathcal{M}} \partial_{t}^{2} u^{f}(\mathbf{x}, t) \varphi_{k}(\mathbf{x}) d V_{g}
$$

Integrating (4.7) by parts, taking into account that all the derivatives of our solution, we obtain the following problem (ODE and initial conditions) for the Fourier coefficients $u_{k}^{f}(t)$ :

$$
\left\{\begin{array}{l}
\frac{d^{2}}{d t^{2}} u_{k}^{f}(t)+\lambda_{k} u_{k}^{f}(t)=-\int_{\partial \mathcal{M}} f(\mathbf{x}, t) \partial_{\nu} \varphi_{k}(\mathbf{x}) d S_{g}, \quad f \in L^{2}\left(\Sigma^{T}\right) \\
u_{k}^{f}(0)=\partial_{t} u_{k}^{f}(0)=0
\end{array}\right.
$$

Solving this ordinary differential equation together with boundary conditions we obtain for smooth $f^{\prime} s$ the following representations:

$$
u_{k}^{f}(t)=\int_{0}^{t} \int_{\partial \mathcal{M}} f\left(\mathbf{z}, t^{\prime}\right) s_{k}\left(t-t^{\prime}\right) \partial_{\nu} \varphi_{k}(\mathbf{z}) d S_{g}(\mathbf{z}) d t^{\prime},
$$




$$
s_{k}(t)= \begin{cases}\frac{\sin \sqrt{\lambda_{k} t}}{\sqrt{\lambda_{k}}}, & \lambda_{k}>0 \\ t, & \lambda_{k}=0 \\ \frac{\sinh \sqrt{\left|\lambda_{k}\right| t}}{\sqrt{\left|\lambda_{k}\right|}}, & \lambda_{k}<0\end{cases}
$$

where $d S_{g}$ is the volume elements of $\partial \mathcal{M}$ which is induced by the metric $g$. Using $u_{k}^{f}(t) \in C([0, T])$ if $f \in L^{2}\left(\Sigma^{T}\right)$ and as even $C_{0}^{\infty}\left(\Sigma^{T}\right)$ is dense $L^{2}\left(\Sigma^{T}\right)$, representation (4.9) is valid for any $f \in L^{2}\left(\Sigma^{T}\right)$.

Result 4.1.2.1 Given the $\operatorname{BSD}(\partial \mathcal{M})$ the Laplace-Beltrami operator and $d S_{g}$ it is possible to find Fourier coefficients of any $u^{f}(t)$.

Let $u^{f}(t)$ and $u^{h}(t)$ be the solutions of (4.5) with $f, h \in L^{2}\left(\Sigma^{T}\right)$, then for any $0 \leq$ $t, s \leq T$ the inner products of the waves may be found by formula:

$$
\left\langle u^{f}(t), u^{h}(s)\right\rangle=\sum_{k=1}^{\infty} u_{k}^{f}(t) \overline{u_{k}^{h}(s)}
$$

where the Fourier coefficients can be found by formula (4.9).

Result 4.1.2.2 (Inner product of two waves) We can find the inner products of any two waves only via the boundary spectral data on $(\partial \mathcal{M})$, for $\eta \in C^{\infty}(\partial \mathcal{M}), \eta>$ $0, d \mu(\mathbf{z})=\eta(\mathbf{z}) d S_{g}$ is some positive boundary measure, $\mathbf{z} \in \partial \mathcal{M}$ :

$$
\begin{array}{r}
<u^{\eta f}(t), u^{\eta h}(s)>=\sum_{k=1}^{\infty} u_{k}^{\eta f}(t) \overline{u_{k}^{\eta h}(s)} \\
\left.u_{k}^{\eta f}(t)=\int_{0}^{t} \int_{\partial \mathcal{M}} f\left(\mathbf{z}, t^{\prime}\right) s_{k}\left(t-t^{\prime}\right) \partial_{\nu} \varphi_{k}(\mathbf{z})\right) d \mu d t^{\prime}
\end{array}
$$




\subsubsection{Domains of influence. Complete system of functions.}

Tataru's theorems. Wave basis.

Orthogonal projectors

Let $\Gamma \subset \partial M$ be non-empty open set,

$$
\begin{aligned}
L^{2}(\Gamma \times[0, T]) & =\left\{f \in L^{2}\left(\Sigma^{T}\right): \operatorname{supp} f \subset \vec{\Gamma} \times[0, T]\right\} \\
L^{2}(U) & =\left\{f \in L^{2}(\mathcal{M}): \operatorname{supp} f \subset \bar{U}\right\}
\end{aligned}
$$

Definition 4.2 (The domain of influence)

Let

$$
\mathcal{M}(\boldsymbol{y}, \tau)=\{x \in \mathcal{M}: d(\boldsymbol{x}, \boldsymbol{y}) \leqslant \tau\}
$$

be called the domain of influence of point $y \in \partial \mathcal{M}$ of time $\tau$, where $d(\boldsymbol{x}, \boldsymbol{y})$ is the distance between $\boldsymbol{x}, \boldsymbol{y}$ in $(\mathcal{M}, g)$. Let

$$
\mathcal{M}(\Gamma, \tau)=\{x \in \mathcal{M}: d(x, \Gamma) \leqslant \tau\}
$$

be called the domain of influence of subset $\Gamma \subset \partial \mathcal{M}$.

The following result is obtained in [38].

Result 4.1.3.1 Let $u^{f}(t)$ be the solution of (4.5), let $f \in L^{2}(\Gamma \times[0, T])$, then

$$
\operatorname{supp}\left(u^{f}(\tau)\right) \subset \mathcal{M}(\Gamma, \tau)
$$

Then

$$
u^{f}(\tau) \in L^{2}(\mathcal{M}(\Gamma, \tau))
$$

here

$$
L^{2}(\Omega)=\left\{f \in L^{2}(\mathcal{M}): f=0 \quad \text { in } \quad \mathcal{M} \backslash \Omega, \quad \Omega \subset \mathcal{M}\right\}
$$


Consider the space of the waves $u^{f}(t), \quad f \in L^{2}(\Gamma \times[0, T])$. It is a linear subspace of $L^{2}(\mathcal{M}(\Gamma, \tau))$. The following results are important for the construction procedure.

Let

$$
u \in H^{1}(\mathcal{M} \times[-T, T]), u=u(\mathrm{x}, t)
$$

be a weak solution of the hyperbolic equation

$$
\square_{g} u+q u=0, \quad \text { in } \mathcal{M} \times[-T, T]
$$

Assume that the Cauchy data of $u$ vanish on $\Gamma \times[-T, T]$,

$$
\left.u\right|_{\Gamma \times[-T, T]}=0, \quad \text { and }\left.\quad \partial_{\nu} u\right|_{\Gamma \times[-T, T]}=0,
$$

where $\Gamma \subset \partial \mathcal{M}$ is an open set.

\section{Definition 4.3 (double cone of influence)}

Let $K \subset \mathcal{M} \times[-T, T]$ be the double cone of influence of $\Gamma \times[-T, T]$,

$$
K=K_{\Gamma, T}=\{(x, t) \in \mathcal{M} \times[-T, T]: d(x, \Gamma) \leq T-|t|\} .
$$

The first Theorem 4.1 is a famous result of D. Tataru, [56], the proof can be found in [38] (see also [57], [58], [34], [33], [49]).

Theorem 4.1 (Tataru's theorem) Assume that coefficients of the d'Alambert operator $\square_{g}$ are from $C^{\infty}\left(B_{\delta}^{\prime}\right)$ and $\left[g^{j k}(x)\right]$ is a real, symmetric, positive definite matrix, $B_{\delta}^{\prime}$ is a ball of radius $\delta$ in local coordinates chart $U^{\prime}$. Assume, in addition, that the surface $\Gamma \in B_{\delta}$ is non-characteristic. Then if $u \in H^{1}\left(B_{\delta}\right)$ is a solution of the wave equation

$$
\left(\square_{g}+q\right) u=0, \quad \text { in } \quad U^{\prime} \times[-\delta, \delta]
$$

which is equal to 0 on one side of $\Gamma$, i.e. $y=(x, t)=\left(y^{1}, y^{0}\right) \in \mathbb{R}^{n+1} ; y^{1}=\left(y^{1}, . ., y^{n}\right)$,

$$
\operatorname{supp}(u) \subset\{\boldsymbol{y}: \psi(\boldsymbol{y}) \leq 0\}, \Gamma=\{\boldsymbol{y}: \psi(\boldsymbol{y})=0\}
$$

then $\operatorname{supp}(u) \cap \Gamma=\varnothing$. 
Theorem 4.2 (Global Holmgren-John Uniqueness theorem) Let $u=u(x, t)$ such that $u \in H^{1}(\mathcal{M} \times[-T, T])$, be a weak solution of the hyperbolic equation (4.14) with (4.15). Then $u$ vanish in double cone $K_{\Gamma, T}$.

Theorem 4.3 (Local unique continuation result) Let

$$
u \in C\left([0,2 \tau] ; H^{1}(\mathcal{M})\right) \cap C^{1}\left([0,2 \tau] ; L^{2}(\mathcal{M})\right)
$$

be a solution in $Q^{2 r}$ of the wave equation $\square_{g} u=0$, such that for an open set $\Gamma \subset \partial \mathcal{M}$

$$
\left\{\begin{array}{l}
\left.u\right|_{\Gamma \times[0,2 \tau]}=0 \\
\left.\partial_{\nu} u\right|_{\Gamma \times[0,2 \tau)}=0 .
\end{array}\right.
$$

Then, at time $t=\tau$ the function $u$ and its derivative $\partial_{t} u$ vanish in the domain of influence of $\Gamma$,

$$
u(\boldsymbol{x}, \tau)=0, \quad \partial_{t} u(\boldsymbol{x}, \tau)=0 \quad \text { for } \quad \boldsymbol{x} \in \mathcal{M}(\Gamma, \tau)
$$

Theorem 4.4 For any $\tau>0$ the linear subspace $\left\{u^{f}(t) \in L^{2}(\mathcal{M}(\Gamma, \tau)): \quad f \in\right.$ $\left.L^{2}(\Gamma, \tau)\right\}$ is dense in $\left.L^{2}(\mathcal{M}(\Gamma, \tau))\right)$.

Lemma 4.1 Let $\tau>0$. Given the BSD it is possible to construct boundary sources $f_{j} \in L^{2}(\Gamma \times[0, \tau])$ such that

$$
v_{j}=u^{\eta f_{j}}(\tau), j=1,2, \ldots
$$

form an orthonormal basis of $L^{2}(\mathcal{M}(\Gamma, \tau))$.

The proof of the latter lemma and theorem 4.2 can be found in section 3.4 of [38], the proof of theorem 4.3 follows from that proof. So, as a result of Lemma 4.1 and Theorem 4.4, we have $\left\{u^{\eta f}(\tau) \in L^{2}(\mathcal{M}(\Gamma, \tau)): \quad f \in L^{2}(\Gamma \times[0, \tau])\right\}$ is also dense in $L^{2}(\mathcal{M}(\Gamma, \tau))$. Thus we can choose a complete set of functions $f_{j} \in C_{0}^{\infty}(\Gamma \times[0, T]), j=$ $1,2 \ldots$, such that $\left\{u^{\eta f_{j}}(\tau)\right\}_{j=1}^{\infty}$ form an orthonormal basis in space $L^{2}(\mathcal{M}(\Gamma, \tau))$. 
Remark 4.1.1 (Gramm-Schmidt orthogonalization) Let us choose a complete set of functions $\left\{h_{j}\right\}_{j=0}^{\infty}$ in $L^{2}(\Gamma \times[0, T])$. Then

$$
\varrho_{j}=h_{j}-\sum_{k=1}^{j-1}\left\langle u^{\eta h_{j}}(\tau), u^{\eta f_{k}}(\tau)\right\rangle f_{k} .
$$

Then we obtain the required for the wave basis functions:

$$
f_{j}=\frac{\varrho_{j}}{\left\langle u^{\eta \varrho_{j}}(\tau), u^{\eta \varrho_{j}}(\tau)\right\rangle^{1 / 2}}
$$

We have got the orthonormalized wave basis.

Notation 4.2 (Orthogonal Projector) Let $P_{\Gamma, \tau}: L^{2}(\mathcal{M}(\Gamma, \tau))$ be the orthogonal projector in $L^{2}(\mathcal{M})$ onto $L^{2}(\mathcal{M}(\Gamma, \tau))$, then $\left(P_{\Gamma, \tau} a\right)(\mathbf{x})=\chi_{\mathcal{M}(\Gamma, \tau)}(\mathbf{x}) a(\mathbf{x})$, where $\chi_{\mathcal{M}(\Gamma, \tau)}(\mathrm{x})$ is the characteristic function such that:

$$
\chi_{\mathcal{M}(\Gamma, \tau)}(\mathbf{x})= \begin{cases}1, & \mathrm{x} \in \mathcal{M}(\Gamma, \tau) \\ 0, & \mathrm{x} \in \mathcal{M}(\Gamma, \tau)\end{cases}
$$

Then $P_{\mathbf{y}, \tau}$ is the orthogonal projector onto $L^{2}(\mathcal{M}(\mathbf{y}, \tau))$.

Result 4.1.3.2 Given two boundary source functions, say, $f, h \in L^{2}\left(\Sigma^{T}\right)$, and given $\Gamma \subset \partial \mathcal{M}$ is an open subset of the boundary, $y \in \partial \mathcal{M}$ is the boundary point, then from the boundary spectral data for any $0 \leqslant t, s, \tau$ we can obtain :

$$
\begin{aligned}
& \left\langle P_{\Gamma, \tau} u^{\eta f}(t), u^{\eta h}(s)\right\rangle=\int_{\mathcal{M}(\Gamma, \tau)} u^{\eta f}(\mathbf{x}, t) \overline{u^{\eta h}(\mathbf{x}, s)} d V_{g} \\
& \left\langle P_{\mathbf{y}, \tau} u^{\eta f}(t), u^{\eta h}(s)\right\rangle=\int_{\mathcal{M}(\mathbf{y}, \tau)} u^{\eta f}(\mathbf{x}, t) \overline{u^{\eta h}(\mathbf{x}, s)} d V_{g} \\
& \left\langle P_{\mathbf{y}, \tau} \varphi_{k}, u^{\eta f}(t)\right\rangle=\sum_{j=1}^{\infty}\left\langle\varphi_{k}, u^{\eta f_{j}}(\tau)\right\rangle\left\langle u^{\eta f}(\tau), u^{\eta f_{j}}(t)\right\rangle .
\end{aligned}
$$

Due to (4.23) and Result 4.1.2.2, we can get all these inner products of the waves given just the boundary spectral data and manifold boundary $\partial \mathcal{M}$. But we don't know yet the boundary measure $\mu=\eta d S_{g}$. 


\subsubsection{On the role of Gaussian beams and boundary distance func- tions}

\section{Definition 4.4 (Geodesic)}

We call the path $\mu([a, b]) \rightarrow \mathcal{M}$ a geodesic if for any $a_{1}, b_{1} \in[a, b]$ with sufficiently small $\left|b_{1}-a_{1}\right|$ the path $\mu\left(\left[a_{1}, b_{1}\right]\right)$ is a shortest path between its endpoints, i.e. arclength $\left|\mu\left(\left[a_{1}, b_{1}\right]\right)\right|=d\left(\mu\left(a_{1}\right), \mu\left(b_{1}\right)\right)$.

Denote a geodesic path $\mu$ by $\gamma$ and parameterize $\gamma$ with its arclength $s$ from a point $\mathbf{y}=\mu(a)$, so that $|d \gamma / d s|_{g}=1$. Let $\mathbf{x}(s)=\left(x^{1}(s), \ldots, x^{m}(s)\right)$ be the representation of $\gamma$ in local coordinates, then $\mathrm{x}(s)$ satisfies the second-order differential equations

$$
\frac{d^{2} x^{k}(s)}{d s^{2}}=-\Gamma_{i j}^{k}(\mathbf{x}(s)) \frac{d x^{i}(s)}{d s} \frac{d x^{j}(s)}{d s}
$$

where

$$
\Gamma_{i j}^{k}(\mathbf{x})=\frac{1}{2} g^{k p}\left(\frac{\partial g_{j p}}{\partial x^{i}}+\frac{\partial g_{i p}}{\partial x^{j}}-\frac{\partial g_{i j}}{\partial x^{p}}\right)
$$

are the Christoffel symbols. Equations (4.24) supplemented with the initial conditions:

$$
x(0)=\mathbf{y} \in \mathcal{M}, \frac{d x(0)}{d s}=\mathbf{w} \in T_{\mathbf{y}} \mathcal{M},|\mathbf{w}|_{g}=1
$$

determine the unique geodesic $\gamma_{\mathbf{y}, \mathbf{w}}$ that starts at the point $\mathbf{y}$ in the direction $\mathbf{w}$.

From now on in this section let $\mathbf{y} \in \partial \mathcal{M}$ be the boundary point, and $\nu$ be the unit inward normal from the boundary point $y \in \partial \mathcal{M}$.

\section{Definition 4.5 (A Critical Value)}

There is a critical value $\tau(z)=\tau_{\partial \mathcal{M}}(z)$ of the geodesic $\gamma_{z, \nu}(t)$, such that for $t<$ $\tau_{\partial \mathcal{M}}(z)$, the geodesic $\gamma_{z, \nu}([0, t])$ is the unique shortest geodesic $\gamma_{z, \nu}(t)$ to $\partial \mathcal{M}$ and, for $t>\tau_{\partial \mathcal{M}}(z)$, it is no more the shortest one. By $l\left(z_{0}\right)$ we denote the maximal arclength of the normal geodesic, which starts at point $z_{0} \in \partial \mathcal{M}$ until it hits the boundary.

Clearly, $l\left(\mathbf{z}_{0}\right)>\tau_{\partial \mathcal{M}}\left(\mathbf{z}_{0}\right)$. 
Gaussian beams can be used to find the distance between any point on a normal geodesic and any boundary point. For any $\mathbf{z}_{0}, \mathbf{y} \in \partial \mathcal{M}$ and $s \in\left[0, l\left(\mathbf{z}_{0}\right)\right]$ we can have the following results. Let the geodesic starts at $\mathbf{z}_{0}$ and be outward normal to the boundary. Denote $U^{f}(\varepsilon ; \mathbf{z}, t)$ Gaussian beam corresponding to that geodesic the solution of the following system (see (3.2)):

$$
\left\{\begin{array}{l}
\partial_{t}^{2} U-\Delta_{g} U=0 \\
\left.U\right|_{t=0}=\left.\partial_{t} U\right|_{t=0}=0 \\
\left.U\right|_{\partial \mathcal{M}}=f(\varepsilon ; \mathbf{z}, t)
\end{array}\right.
$$

where $f(\varepsilon ; \mathbf{z}, t)=(\pi \varepsilon)^{-\frac{m}{4}} \chi(\mathbf{z}, t) \exp \left\{i \varepsilon^{-1} \Theta(\mathbf{z}, t)\right\} V(\mathbf{z})$, see (3.3). Let $\mathbf{z}_{0}, \mathbf{y} \in \partial \mathcal{M}$, $t_{0}>0, \mathrm{z}$ be the local coordinate system on $\partial \mathcal{M}$. Let $U_{0,0}^{f}\left(t_{0}\right)=V\left(\mathbf{z}_{0}\right)$, and then

$$
U_{0,0}^{f}(t)=\operatorname{det}(Y(t))^{\frac{1}{2}}\left[\frac{g\left(\mathbf{z}_{0}, 0\right)}{g\left(\mathbf{z}_{0}, t-t_{0}\right)}\right]^{\frac{1}{4}} V\left(\mathbf{z}_{0}\right) .
$$

Let $U^{f}(\varepsilon ; t)$ be the Gaussian beam propagating along the normal geodesic, and so does the wave $U^{\eta f}(\varepsilon ; t)$ corresponding to the boundary source $\eta f(\varepsilon ; \cdot)$. We have the following result:

Lemma 4.2 For any $\Gamma \subset \partial \mathcal{M}, t_{0}<t<t_{0}+l\left(z_{0}\right)$ and $\tau_{1}>0$,

$$
\lim _{\varepsilon \rightarrow 0}\left\langle P_{\Gamma, \tau_{1}} U_{\varepsilon}(\cdot, t), U_{\varepsilon}(\cdot, t)>= \begin{cases}\alpha\left(z_{0}\right), & \text { if } x(t) \in \mathcal{M}^{\text {int }}\left(\Gamma, \tau_{1}\right), \\ 0, & \text { if } x(t) \in \mathcal{M} \backslash \mathcal{M}\left(\Gamma, \tau_{1}\right),\end{cases}\right.
$$

where

$$
\alpha\left(z_{0}\right)=\frac{\left|V\left(z_{0}\right)\right|^{2}\left[g\left(z_{0}, 0\right)\right]^{\frac{1}{2}}}{\sqrt{\operatorname{det}(\Im H(t))}|\operatorname{det}(Y(t))|}=\frac{\left|V\left(z_{0}\right)\right|^{2}\left[g\left(z_{0}, 0\right)\right]^{\frac{1}{2}}}{\sqrt{\operatorname{det}(\Im H(0))}|\operatorname{det}(Y(0))|}>0 .
$$

Then as $V(z)=\rho(z)$ for the Gaussian beam $U^{\eta f}(\varepsilon ; t)$ we have for $s<l\left(z_{0}\right)$ :

$$
\lim _{\varepsilon \rightarrow 0}\left\|P_{\boldsymbol{y}, \tau} U^{\eta f}\left(\varepsilon ; s+t_{0}\right)\right\|= \begin{cases}\left|\eta\left(z_{0}\right)\right|^{2}\left(g\left(z_{0}, 0\right)\right)^{\frac{1}{2}} h_{Y}(t), & d\left(\gamma_{z_{0}, \nu}(s), y\right)<\tau \\ 0, & d\left(\gamma_{z_{0}, \nu}(s), y\right)>\tau\end{cases}
$$

where $\left.h_{Y}=[\operatorname{det}(\Im H(t)))\right]^{\frac{-1}{2}}|\operatorname{det}(Y(t))|^{-1}$. As $\left|\eta\left(z_{0}\right)\right|^{2}\left(g\left(z_{0}, 0\right)\right)^{\frac{1}{2}} h_{Y}(t)$ is strictly positive, we can find $d\left(\gamma_{z_{0}, \nu}, y\right)$. 
Thus the boundary spectral data uniquely determines $d\left(\gamma_{\mathbf{z}_{0}, \nu}(s), \mathbf{y}\right)$, where $\nu(s)$ is a normal to the boundary at point $z_{0} \in \partial \mathcal{M}, \gamma$ is the geodesic, going from $z_{0}$ in the direction of the normal $\nu$.

Result 4.1.4.1 (Distance to the boundary) Minimizing $\left\{\mathbf{y} \rightarrow d\left(\gamma_{\mathbf{z}_{0}, \nu}(s), \mathbf{y}\right)\right\}$ we can find $d\left(\gamma_{\mathrm{z}_{0}, \nu}(s), \partial \mathcal{M}\right)$. So, we know the distance from $\gamma_{z_{0}, \nu}$ to the boundary and nearest points on $\partial \mathcal{M}$ to $\gamma_{z_{0}, \nu}(s)$.

By increasing $s$ we can find the arclength $l\left(\mathbf{z}_{0}\right)$, when the geodesic $\gamma_{\mathbf{z}_{0}, \nu}(s)$ hits the boundary for the first time. Let us denote by

$$
\tau_{\partial \mathcal{M}}\left(\mathbf{z}_{0}\right)=\sup _{s \geq 0}\left\{s \mid d\left(\gamma_{\mathbf{z}_{0}, \nu}(s), \mathbf{z}_{0}\right)=d\left(\gamma_{\mathbf{z}_{0}, \nu}(s), \partial \mathcal{M}\right)\right\}
$$

This is the maximal time for which you can go from the boundary point $\mathbf{z}_{0}$ in the direction $\nu$ and this point $\mathrm{z}_{0}$ will be still the nearest point from the boundary.

Notation 4.3 (Boundary distance functions) The boundary distance function is determined as the distance from an arbitrary fixed point $\mathrm{x} \in \mathcal{M}$ to any point of the boundary $r_{\mathbf{x}}: \partial \mathcal{M} \rightarrow \overline{\mathbf{R}}_{+}$as $r_{\mathbf{x}}(\mathbf{y})=d(\mathbf{x}, \mathbf{y}), \quad \mathbf{y} \in \partial \mathcal{M}$. We will call $\mathcal{R}: \mathcal{M} \rightarrow$ $L^{\infty}(\partial \mathcal{M}), \quad \mathrm{x} \rightarrow r_{\mathbf{x}}$ the function which assigns to any point $\mathrm{x} \in \mathcal{M}$ the corresponding boundary distance function, i.e. $r_{\mathbf{x}}(\mathrm{y})=d(\mathrm{x}, \mathrm{y}), \mathrm{y} \in \partial \mathcal{M}$.

Notation 4.4 (The set of boundary distance functions) Let

$$
\mathcal{R}(\mathcal{M})=\left\{r_{\mathbf{x}} \in L^{\infty}(\partial \mathcal{M}): \mathbf{x} \in \mathcal{M}\right\}
$$

be the set of boundary distance functions.

The norm of $L^{\infty}(\mathcal{M})$ is $\|r\|_{L^{\infty}}=\sup _{\mathbf{z} \in \partial \mathcal{M}}|r(\mathbf{z})|$. We know $d\left(\gamma_{\mathbf{z}, \nu}(s)\right.$,y) for every $s \in$ $\left[0, \tau_{\partial \mathcal{M}}(\mathbf{z})\right]$ thus we can find

$$
r_{\mathbf{z}, s}=d\left(\gamma_{\mathbf{z}, \nu}(s), \mathbf{y}\right), \mathbf{y} \in \partial \mathcal{M} \text {, i.e. } r_{\mathbf{z}, s}=r_{\mathbf{x}} \text { for } \mathbf{x}=\gamma_{\mathbf{z}, \nu}(s)
$$


Result 4.1.4.2 The boundary spectral data $\left\{\partial \mathcal{M}, \lambda_{j},\left.\partial_{\nu} \varphi_{j}\right|_{\partial \mathcal{M}}, j=1,2, ..\right\}$ determine the set $\mathcal{R}(\mathcal{M})=\left\{r_{\mathbf{z}, s} \in L^{\infty}(\partial \mathcal{M}): \mathbf{z} \in \partial \mathcal{M}, s \in\left[0, \tau_{\partial \mathcal{M}}(\mathbf{z})\right]\right\}$.

Remark 4.1.2 (Manifold reconstruction) In order to reconstruct differentiable manifold $(\mathcal{M}, g)$, we can determine $(\mathcal{R}(\mathcal{M}), \widetilde{g})$, so that $\mathcal{R}$ becomes an isometry. Just the knowledge of $\mathcal{R}(\mathcal{M}) \subset L^{\infty}(\partial \mathcal{M})$ is sufficient to find the differentiable and Riemannian structures on $\mathcal{M}$. The mapping $\mathcal{R}: \mathcal{M} \rightarrow \mathcal{R}(\mathcal{M})$ is a homeomorphism. As this fails completely for a Riemannian polyhedron we omit this part of the reconstruction in this thesis. In [38] it is shown that supplying the $\mathcal{R}(\mathcal{M})$ by the structure of differentiable manifold is enough to make it diffeomorphic to $\mathcal{M}$.

Result 4.1.4.3 (Boundary measure) Using the boundary spectral data, and applying results of part two of the (4.23), we can determine uniquely $\left\|u_{\varepsilon, \rho}(\cdot, t)\right\|$. Then we can evaluate

$$
\lim _{\varepsilon \rightarrow 0}\left\|u_{\varepsilon, \eta}(t)\right\|^{2}=\frac{\left|\eta\left(\mathbf{z}_{0}\right)\right|^{2} \sqrt{g\left(\mathbf{z}_{0}, 0\right)}}{\sqrt{\operatorname{det}(\Im H(t))}|\operatorname{det}(Y(t))|},
$$

using (4.31). Now as metric $g$ is known (see Remark 4.1.2), recalling that $H_{0}$ is in our disposal, then we can find $\Im H(t), Y(t)$ (see Lemma 3.1). Then we can find $\eta\left(\mathbf{z}_{0}\right)>0$. Thus we found $d \mu=\eta d S_{g}$. 


\subsection{IP with data given on a part of the boundary}

In this section we describe briefly the method used in [38] to prove Statement 4.1.1.1 for the boundary spectral data given on a part $\Gamma \subset \partial \mathcal{M}$. We will describe a procedure of constructing an isometric copy of $(\mathcal{M}, g)$.

Remark 4.2.1 The construction of $\mathcal{M}$ will be given by iterating local constructions.

\subsubsection{First submanifold reconstruction}

First, we construct the manifold $\mathcal{M}$ near the given set $\Gamma \subset \partial \mathcal{M}$. Let $\mathrm{z} \in \Gamma$ and $\gamma_{\mathrm{z}, \nu}$ be the normal geodesic starting at $\mathbf{z}$. The analogue of the function $\tau_{\partial \mathcal{M}}$, is the following upper semicontinuous function:

$$
\tau_{\Gamma}(\mathbf{z})=\left\{\sup s>0: d\left(\gamma_{\mathbf{z}, \nu}(s), \Gamma\right)=s\right\}
$$

Let

$$
\Omega_{\Gamma}=\left\{(\mathbf{z}, s) \in \Gamma \times \mathbb{R}_{+}: s<\tau_{\Gamma}(\mathbf{z})\right\}
$$

be the largest open set that lies under the graph of $\tau_{\Gamma}$. Clearly, $\Gamma \times\left(0, \inf _{\mathbf{z} \in \Gamma} \tau_{\partial \mathcal{M}}\right) \subset \Omega_{\Gamma}$. The mapping

$$
\exp _{\partial \mathcal{M}}: \Omega_{\Gamma} \rightarrow \mathcal{M}
$$

is a diffeomorphism between $\Omega_{\Gamma}$ and $\mathcal{M}_{\Gamma}$,

$$
\mathcal{M}_{\Gamma}=\exp _{\partial \mathcal{M}}\left(\Omega_{\Gamma}\right) \subset \mathcal{M}
$$

Let $\widetilde{g}=\left(\exp _{\partial \mathcal{M}}\right)_{*} g$ be the metric on $\Omega_{\Gamma}$, so that $\exp _{\partial \mathcal{M}}$ is an isometry between $\left(\Omega_{\Gamma}, \widetilde{g}\right)$ and $\left(\mathcal{M}_{\Gamma}, g\right)$. In the following we denote the first step of iteration

$$
\mathcal{M}^{1}=\mathcal{M}_{\Gamma}
$$

Our first aim is to construct $\left(\Omega_{\Gamma}, \widetilde{g}\right)$. Consider the inverse boundary value problem (4.5). As before, we will use an arbitrary positive smooth measure $d \mu$ on $\Gamma$. Then 
there exists a function $\eta \in C^{\infty}(\partial \mathcal{M}), \eta>0$, such that $d \mu=\eta d S_{g}$. Using results of subsection 4.1.2.2 we can compute the inner product

$$
\int_{\mathcal{M}} u^{\eta f}(t) \overline{u^{\eta h}(s)} d V_{g}
$$

from the boundary spectral data on $\Gamma$ for any $f, h \in C_{0}^{\infty}(\Gamma \times(0, \infty))$ and $t, s \geq 0$. At this stage function $\eta$ is still unknown. We obtain results similar to Theorem 4.2 and (4.23):

- Let $\Gamma_{1} \subset \Gamma$ be an open set and $\tau>0$. Given the boundary spectral data on $\Gamma$, it is possible to construct boundary sources $f_{j} \in C_{0}^{\infty}(\Gamma \times(0, \infty)), j=1,2, \ldots$ such that

$$
v_{j}=u^{\eta f_{j}}(\tau)
$$

form an orthonormal basis of $L^{2}\left(\mathcal{M}\left(\Gamma_{1}, \tau\right)\right)$.

- Let $f, h \in C_{0}^{\infty}(\Gamma \times(0, \infty))$ and $\Gamma_{1} \subset \Gamma$ be an open set. Then, given the boundary spectral data on $\Gamma$, it is possible to find the inner product

$$
<P_{\Gamma_{1}, \tau} u^{\eta f}(t), u^{\eta h}(s)>=\int_{\mathcal{M}\left(\Gamma_{1}, \tau\right)} u^{\eta f}(\mathbf{x}, t) \overline{u^{\eta h}(\mathbf{x}, s)} d V_{g},
$$

for any $t, s, \tau>0$.

Now we need to construct the function $\tau_{\Gamma}$. To this end, we observe that, for $\Gamma_{1} \subset$ $\Gamma, s, t>0$ we have $\mathcal{M}\left(\Gamma_{1}, s\right) \subset \mathcal{M}(\Gamma, t)$ if and only if $\left\|P_{\Gamma, \tau} u^{\eta f}(s)\right\|=\left\|u^{\eta f}(s)\right\|$ for all $f \in C_{0}^{\infty}\left(\Gamma_{1} \times(0, s)\right)$. This is the effect of Lemma 4.4. On the other hand, $s<\tau_{\Gamma}\left(\mathbf{z}_{0}\right)$ if and only if, for any $t<s$ and neighborhood $\Gamma_{1} \subset \Gamma$ of $\mathrm{z}_{0}$, we have $\mathcal{M}\left(\Gamma_{1}, s\right) \nsubseteq \mathcal{M}(\Gamma, t)$. Hence, the boundary spectral data on $(\Gamma)$ determine $\tau_{\Gamma}$ and, therefore, $\Omega_{\Gamma}$. Using local coordinates $\mathbf{z}=\left(z^{1}, . ., z^{(n-1)}\right)$ on $\Gamma$, we obtain local coordinates $\left(z^{1}, . ., z^{(n-1)}, s\right)$ on $\Omega_{\Gamma}$. To construct the metric $\tilde{g}$ on $\Omega_{\Gamma}$, we use the technique of Gaussian beams.

As we have shown in the previous section, we see that if $\mathrm{z}, \mathrm{y} \in \Gamma$ and $(\mathrm{y}, s) \in \Omega_{\Gamma}$, then boundary spectral data on $\Gamma$ determine $d\left(\gamma_{\mathbf{y}, \nu}(s), \mathbf{z}\right)$. 
Thus we are able to construct the analogs of the evaluation functions $E(\mathbf{z})$, i.e., the functions

$$
\begin{gathered}
E_{\mathbf{z}}^{\Gamma}: \Omega_{\Gamma} \rightarrow \mathbb{R}, \\
E_{\mathbf{z}}^{\Gamma}(\mathbf{y}, s)=d\left(\mathbf{z}, \gamma_{\mathbf{y}, \nu}(s)\right),
\end{gathered}
$$

where $\mathbf{z} \in \Gamma$. Evaluating differentials $d_{\mathbf{y}, s} E_{\mathbf{z}}^{\Gamma}$ at a point $\left(\mathbf{y}_{0}, s_{0}\right) \in \Omega_{\Gamma}$ and using the same considerations as in section 4.1 , with $r$ replaced by $(y, s)$, we can find the metric tensor $\widetilde{g_{i j}}\left(\mathbf{y}_{0}, s_{0}\right)$. As $\left(\Omega_{\Gamma}, \widetilde{g}\right)$ is isometric to $\left(\mathcal{M}_{\Gamma}, g\right)$, we obtain the following results:

Result 4.2.1.1 (Subset $\mathcal{M}_{\Gamma}$ reconstruction) Let the boundary spectral data on $\Gamma$ be given, then it is possible to construct the Riemannian manifold $\left(\mathcal{M}_{\Gamma}, g\right)$.

Result 4.2.1.2 (Inner products) As the metric tensor $g$ on $\mathcal{M}_{\Gamma}$ (and, therefore, $d S_{g}$ ) are already found, we can find the function $\eta$ (see Result 4.1.2 for the procedure). Thus for any $f, h \in C_{0}^{\infty}(\Gamma \times(0, \infty))$ and any $t, s \geqslant 0$, we can evaluate the inner product

$$
\int_{\mathcal{M}} u^{f}(t) \overline{u^{h}(s)} d V_{g} .
$$

The boundary spectral data of $(\Delta, \Gamma)$ determine uniquely the restrictions on $\mathcal{M}_{\Gamma}$ of eigenfunctions $\varphi_{j}, j=1,2, \ldots$.

\subsubsection{Recalculation of the boundary spectral data of $\left(\Delta_{\mathcal{D}}, \partial \mathcal{D}\right)$}

To continue the construction, let $\mathcal{D} \subset \mathcal{M}_{\Gamma}$ be an open domain with smooth boundary $\partial \mathcal{D}$. Consider sub-manifold $\mathcal{M} \backslash \mathcal{D}$ with boundary $\partial(\mathcal{M} \backslash \mathcal{D})=\partial \mathcal{M} \cup \partial \mathcal{D}$. Let $\Delta_{\mathcal{D}}$ be the Dirichlet Laplace-Beltrami operator $-\Delta_{g}$, on $\mathcal{M} \backslash \mathcal{D}$. We are going to find the boundary spectral data of $\left(\Delta_{\mathcal{D}}, \partial \mathcal{D}\right)$.

Lemma 4.3 (Data recalculation) Assume that we are given an open part $\Gamma \subset$ $\partial \mathcal{M}$ and the boundary spectral data of $(\Delta, \Gamma)$. Assume, in addition, that we know the Riemannian manifold $\left(\mathcal{M}_{\Gamma}, g\right)$ and the restrictions of the eigenfunctions $\left.\varphi_{j}\right|_{\mathcal{M}_{\Gamma}}, j=$ $1,2, \ldots$. Then these data determine the boundary spectral data of $\left(\Delta_{\mathcal{D}}, \partial \mathcal{D}\right)$. 
The complete proof of this lemma can be found in [38], or [5] and we omit it here.

\subsubsection{Reconstruction of $\mathcal{M}^{2}$}

In this subsection we complete the proof of Statement 4.1.1.1. Consider again $\mathcal{M} \backslash \mathcal{D}$ and a Laplace-Beltrami operator $\Delta_{\mathcal{D}}$ on it. Then, the boundary spectral data of $\left(\Delta_{\mathcal{D}}, \partial \mathcal{D}\right)$ are given on a part $\partial \mathcal{D}$, of the boundary $\partial(\mathcal{M} \backslash \mathcal{D})$ of $\mathcal{M} \backslash \mathcal{D}$. Using the same constrictions, as in subsection 4.1.1, with $\Gamma$ replaced by $\partial \mathcal{D}$ and $\mathcal{M}$ replaced by $\mathcal{M} \backslash \mathcal{D}$, we find a manifold $\mathcal{M}_{\partial \mathcal{D}} \subset \mathcal{M} \backslash \mathcal{D}$ and the restrictions of metric $g$ and all eigenfunctions $\varphi_{j}^{\mathcal{D}}$ on $\mathcal{M}_{\partial \mathcal{D}}$. Now $\varphi_{j, k}^{\mathcal{D}}$ can be considered in two ways: on one hand, they are the Fourier coefficients of the zero-continuations of the eigenfunctions $\varphi_{j}^{\mathcal{D}}$ with respect to the basis $\left.\varphi_{k}\right|_{\mathcal{M}}$ of $L^{2}(\mathcal{M})$; on the other hand, they are the Fourier coefficients of $\left.\varphi_{k}\right|_{\mathcal{M} \backslash \mathcal{D}}$, i.e.

$$
\varphi_{k}(\mathbf{x})=\sum_{j=1}^{\infty} \varphi_{j, k}^{\mathcal{D}} \varphi_{j}^{\mathcal{D}}(\mathbf{x}), \quad \mathrm{x} \in \mathcal{M} \backslash \mathcal{D} .
$$

As we know $\varphi_{j}^{\mathcal{D}}(\mathbf{x}), \mathbf{x} \in \mathcal{M}_{\partial \mathcal{D}}$, we can find $\varphi_{k}(\mathbf{x})$ in $\mathbf{x} \in \mathcal{M}_{\partial \mathcal{D}}$. So far, for any $\mathcal{D} \subset \mathcal{M}$, we have constructed a manifold $\mathcal{M}_{\partial \mathcal{D}} \subset \mathcal{M} \backslash \mathcal{D}$, and the eigenfunctions $\varphi_{k}, \varphi_{j}^{\mathcal{D}}$, and metric tensor $g$ on it.

Let $\mathcal{D}$ and $\mathcal{D}^{\prime}$ be subsets of $\mathcal{M}$. In the manifolds $\mathcal{M}_{\partial \mathcal{D}}$ and $\mathcal{M}_{\partial \mathcal{D}^{\prime}}$ we identify the points $\mathbf{x} \in \mathcal{M}_{\partial \mathcal{D}}$ and $\mathbf{x}^{\prime} \in \mathcal{M}_{\partial \mathcal{D}^{\prime}}$, such that $\varphi_{j}(\mathbf{x})=\varphi_{j}\left(\mathbf{x}^{\prime}\right)$ for all $j=1,2, \ldots$ In this case, the points $\mathbf{x}$ and $\mathbf{x}^{\prime}$ correspond to the same point on $\mathcal{M}$. Analogously, we identify points on $\mathrm{x} \in \mathcal{M}_{\partial \mathcal{D}}$ and $\mathcal{M}_{\Gamma}=\mathcal{M}^{1}$ that correspond to the same point on $\mathcal{M}$. Using these identifications, we can construct the manifold $\mathcal{M}^{2} \subset \mathcal{M}$,

$$
\mathcal{M}^{2}=\bigcup_{\mathcal{D} \subset \mathcal{M}} \mathcal{M}_{\partial \mathcal{D}} \cup \mathcal{M}^{1}
$$

It also follows from the previous considerations that we have constructed the restrictions of the metric $g$ and the eigenfunctions on these manifolds. Next we show that, for sufficiently large $m$,

$$
\mathcal{M}^{m}=\mathcal{M}^{\text {int }}
$$




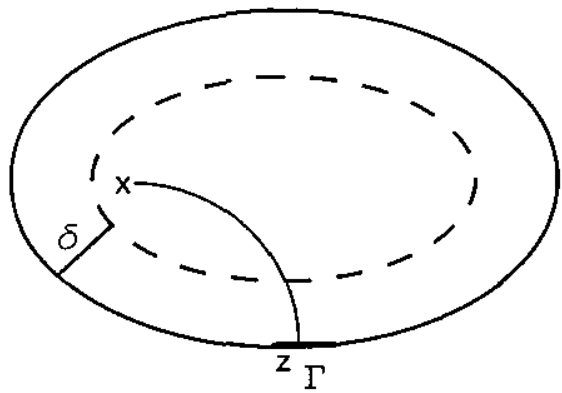

Figure 4.1: We can reach any point on the manifold by path, which does not go into the collar neighborhood of the boundary.

\subsubsection{Iterating procedure; $\mathcal{M}^{m}=\mathcal{M}^{\text {int }}$}

To show (4.42), consider a compact manifold $\mathcal{N}$ without boundary, such that $\mathcal{M} \subset \mathcal{N}$. By compactness of $\mathcal{N}$, there is $\delta>0$ such that

$$
\delta<\min \left\{\min _{\mathbf{z} \in \partial \mathcal{M}} \tau_{\partial \mathcal{M}}(\mathbf{z}), \min _{\mathbf{y}, \mathbf{w} \in S \mathcal{N}} \tau(\mathbf{y}, \mathbf{w})\right\}
$$

here $\tau_{\partial \mathcal{M}}(\mathbf{z})$ and $\tau(\mathbf{y}, \mathbf{w})$ are the critical values of the functions that correspond to the boundary exponential map on $\mathcal{M}$ and the exponential map on $\mathcal{N}, S \mathcal{N}=\{(\mathrm{y}, \mathrm{w}) \in$ $\left.T \mathcal{N}:|\mathbf{w}|_{g}=1\right\} ; \gamma(0)=\mathrm{y} \in \mathcal{N}, \frac{d \gamma(0)}{d s}=\mathrm{w} \in T_{y} \mathcal{N}, \gamma$ is a normal geodesic, see (4.4), (4.26), Figure 4.1. Next, we consider the set

$$
\mathcal{M} \backslash \mathcal{M}^{\delta}=\{x \in \mathcal{M}: d(x, \partial \mathcal{M}) \geq \delta\}
$$

Due to definition (4.43) of $\delta, \mathcal{M} \backslash \mathcal{M}^{\delta}$ is a manifold with smooth boundary that is homotopic to $\mathcal{M}$ and, therefore, connected. Thus there is a constant $T_{\Gamma}>0$, such that any $\mathrm{x} \in \mathcal{M}$ can be connected with $\Gamma$ by a smooth path $\mu \subset \mathcal{M}$ of length $L, L \leq T_{\Gamma}$. Moreover, if $\mu$ is parameterized with its arclength, then the following conditions are satisfied.

i) $\mu(0)=\mathrm{z} \in \Gamma, \mu(L)=\mathrm{x}$, 
ii) $\mu[0, \delta]$ coincides with the normal geodesic to $\mathbf{z}$,

iii) If $d(\mathbf{x}, \partial \mathcal{M}) \leq \delta$, then $\mu[L-(\delta-d(\mathbf{x}, \partial \mathcal{M})), L]$ coincides with the continuation of the normal geodesic from the boundary to the point $\mathrm{x}$,

iv) When $\delta<s<L-(\delta-d(\mathbf{x}, \partial \mathcal{M}))$, then $\mu(s) \in \mathcal{M} \backslash \mathcal{M}^{\delta}$.

Let

$$
\mathbf{x}_{k}=\mu(k \delta), k=1, \ldots, K, K=\left[\frac{L}{\delta}\right], \mathbf{x}_{K+1}=\mathbf{x} .
$$

By previous constructions, $\mathrm{x}_{1} \in \mathcal{M}^{1}$. Assume that $\mathrm{x}_{k} \in \mathcal{M}^{k}, k<K$. Then, for sufficiently small $\rho>0$ and $\mathcal{D}=B_{\rho}\left(\mathrm{x}_{k}\right)$, we have $\mathcal{D} \subset \mathcal{M}^{k}$. It follows from definition (4.43) of $\delta$, that

$$
\inf _{\mathbf{z} \in \partial \mathcal{D}} \tau_{\partial \mathcal{D}}(\mathbf{z})>\delta-\rho
$$

Thus, $B_{\delta}\left(\mathbf{x}_{k}\right) \subset \mathcal{M}^{k+1}$ and, in particular, $\mathbf{x}_{k+1} \in \mathcal{M}^{k+1}$. By induction, we see that $\mathrm{x}=\mathrm{x}_{k+1} \subset \mathcal{M}^{k+1}$, which proves the assertion, that $\mathcal{M}^{m}=\mathcal{M}^{\text {int }}$, for $m$ sufficiently large. Thus we have proved statement 4.1.1.1. 


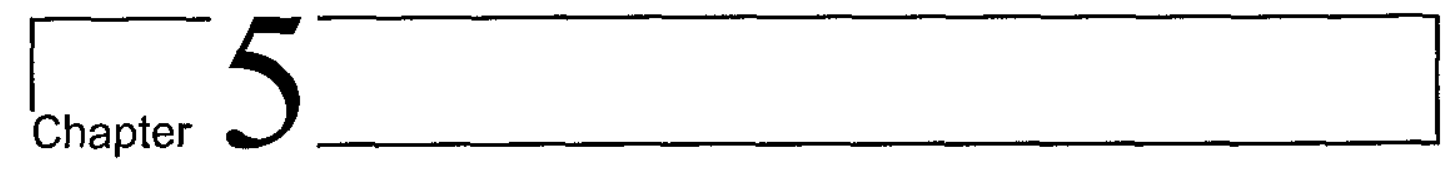

\section{Uniqueness Problem for the Polyhedron}

\subsection{Formulation of the uniqueness problem}

Consider two compact $n$-dimensional $(n \geq 1)$ admissible Riemannian polyhedra (see definition (2.19)) $\mathcal{M}, \widetilde{\mathcal{M}}$ with boundaries $\partial \mathcal{M}$ and $\partial \widetilde{\mathcal{M}}$ correspondingly. We assume, that the Dirichlet Laplace-Beltrami operators $\Delta_{g}, \Delta_{\tilde{g}}$ are defined on them (see Chapter 2). We denote by $\lambda_{k}, \tilde{\lambda}_{k}$ the eigenvalues, $\varphi_{k}, \widetilde{\varphi}_{k}$ the orthonormalized eigenfunctions of $\Delta_{g}, \Delta_{\tilde{g}}$ correspondingly. Thus we can determine the boundary spectral data $\left(\Delta_{g}, \Gamma\right), \Gamma \subset \partial \mathcal{M}$ is an open part of the boundary, and the boundary spectral data $\left(\Delta_{\tilde{g}}, \widetilde{\Gamma}\right)$, here $\widetilde{\Gamma} \subset \partial \widetilde{\mathcal{M}}$ is an open part of the boundary. Thus we can formulate main result of this thesis for them:

Theorem 5.1 (Uniqueness Theorem 1) : Let the sets of boundary spectral data of two polyhedra be equal, i.e.

$$
B S D\left(\Delta_{g}, \partial \mathcal{M}\right)=B S D\left(\Delta_{\tilde{g}}, \partial \widetilde{\mathcal{M}}\right)
$$


or, more precisely,

$$
\left\{\begin{array}{l}
\partial \mathcal{M}=\partial \widetilde{\mathcal{M}} \\
\lambda_{k}=\widetilde{\lambda}_{k} \\
\left.\partial_{\nu} \varphi_{k}\right|_{\partial \mathcal{M}}=\left.\partial_{\widetilde{\nu}} \widetilde{\varphi_{k}}\right|_{\partial \widetilde{\mathcal{M}}} .
\end{array}\right.
$$

Then polyhedron $\mathcal{M}$ is isometric to $\widetilde{\mathcal{M}}$.

Or, in more general case,

Theorem 5.2 (Uniqueness Theorem 2) : Let the sets of boundary spectral data $\left(\Delta_{g}, \Gamma \subset \partial \mathcal{M}\right)$ be isometric to the set of boundary spectral data $\left(\Delta_{\tilde{g}}, \widetilde{\Gamma} \subset \partial \widetilde{\mathcal{M}}\right)$, or , more precisely,

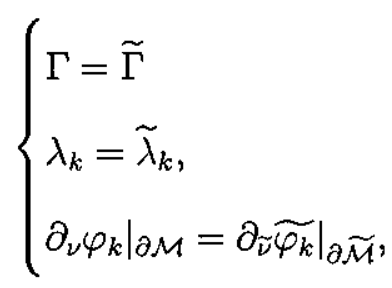

Then polyhedron $\mathcal{M}$ is isometric to $\widetilde{\mathcal{M}}$.

Theorem 5.2 is a piece-wise smooth analog of the statement 4.1.1.1. As Theorem 5.2 is more general than Theorem 5.1, we will concentrate on its proof.

Notation 5.1 As we consider the set of boundary spectral data $\left(\Delta_{g}, \Gamma \subset \partial \mathcal{M}\right)$, and the set of boundary pectral data $\left(\Delta_{\widetilde{g}}, \widetilde{\Gamma} \subset \partial \widetilde{\mathcal{M}}\right)$, we denote them $\operatorname{BSD}(\Gamma)$ and $\operatorname{BSD}(\widetilde{\Gamma})$ respectively.

Remark 5.1.1 (Restrictions on $\Gamma$ ) Without any loss of generality we assume that each of $\Gamma$ and $\widetilde{\Gamma}$ belongs strictly to one chamber, say $\Omega_{1}$ and $\widetilde{\Omega_{1}}$ and does not contain any wedge points. 


\subsection{The Holmgren-John uniqueness theorem}

Consider $\mathcal{M}$ to be an admissible Riemannian polyhedron. Let $\Gamma \subset \partial \mathcal{M}, \Gamma \neq 0$, be an open set,

$$
\Sigma^{2 T}=\Gamma \times(0,2 t), \quad Q^{2 T}=\mathcal{M} \times(0,2 T) .
$$

\section{Definition 5.1 (Weak solution)}

We call function $u \in L_{2}\left(Q^{2 T}\right)$ a weak solution to the Cauchy problem for the wave equation

$$
\square_{g} u=0, \quad Q^{2 T},
$$

with initial data

$$
\left.u\right|_{\Sigma^{2 T}}=\left.\partial_{\nu} u\right|_{\Sigma^{2 T}}=0
$$

if

$$
\int_{Q^{2 T}} u \cdot \square_{g} \psi d x d t=0
$$

for all $\psi \in C^{0, \Delta_{g}}\left(Q^{2 T}, \Sigma_{0}^{2 T}\right)$, where

$$
\begin{gathered}
C^{0, \Delta_{g}}\left(Q^{2 T}, \Sigma^{2 T}\right)=\left\{\psi: \psi \in C^{p}\left((0,2 T), \mathcal{D}\left(\Delta_{g}\right)\right) \cap C^{p+2}\left((0,2 T), L_{2}(\mathcal{M})\right)\right. \\
\left.\operatorname{supp} \psi \cap\left(\partial Q^{2 T} \backslash \Sigma^{2 T}\right)=\emptyset\right\} \\
\mathcal{D}\left(\Delta_{g}\right)=\left\{\xi \in H^{1}(\mathcal{M}): \Delta_{g} \xi \in L_{2}(\mathcal{M})\right\}
\end{gathered}
$$

The cone of influence is

$$
K^{2 T}(\Gamma)=\left\{(\mathbf{x}, t) \in Q^{2 T}:|T-t| \leq T-d_{\mathcal{M}}(\mathbf{x}, \Gamma)\right\}
$$

where

$$
d_{\mathcal{M}}(\mathbf{x}, \Gamma)=\inf _{\mathbf{y} \in \Gamma} d_{\mathcal{M}}(\mathbf{x}, \mathbf{y})
$$

(see Chapter 2), where the infinum is taken over all curves that pass through the interfaces transversally finite number of times such that they do not transverse wedge points $W P^{j}, j \leq n-2$. 
Theorem 5.3 (Holmgren-John uniqueness theorem for $\mathcal{M}=\Omega$ ) Let function $u \in L_{2}\left(Q^{2 T}\right)$ be a weak solution to problem (5.3), (5.4), where $Q^{2 T}=\Omega \times(0,2 T)$, and $\Omega$ is a chamber, the $u \stackrel{\text { a.e }}{=} 0$ in $K^{2 T}(\Gamma)$.

This is Theorem 1 from [45], the exact proof of this theorem can be found there. The proof is based on the fact that $d_{\Omega}(\mathbf{x}, \mathbf{y})$ is continuous for any $\mathbf{x}, \mathbf{y} \in \Omega$. It is shown, that for any point $\mathrm{x} \bar{\epsilon} \partial \Omega$ such that $d_{\Omega}(x, \Gamma)<T$, and for any $\varepsilon>0$ there exists a vicinity $U \ni \mathbf{x}$, such that

$$
u \stackrel{a . e .}{=} 0, \text { with }\left\{(z, t): z \in U,|T-t| \leq T-d_{\Omega}(\mathbf{x}, \Gamma)-\varepsilon\right\}
$$

Due to the results of Chapter 2, there exists a curve $z(s), 0 \leq s \leq s_{0}, z(0)=\mathrm{y} \in$ $\Gamma, z\left(s_{0}\right)=\mathbf{x}$, which is transversal to $\partial \Omega$ at the point $\mathbf{y}$, such that

$$
s_{0} \leq d_{\Omega}(x, \Gamma)+\frac{\varepsilon}{2}
$$

Assume that $\Gamma \subset \Gamma_{r}$, for some $r$, where $\partial \Omega=\cup \Gamma_{i}$. Let $U, \psi$ be the vicinity of the point $\mathrm{y} \in \Gamma$ and the coordinate mapping such that $\psi(\mathrm{y})=0$ and $\psi(U)=\{z \in W: f(z) \geq$ $0\}, f \in C^{1}(\bar{W})$, and without loss of generality, $\partial f / \partial q^{\alpha}(0)=0, \alpha=1, . ., n-1$, $z=\left(q^{\alpha}, \sigma\right)$. As $g_{i l} \in C^{p}(\overline{\psi(U)})$, there exists an open vicinity $V$, such that $0 \in V \subset W$ such that $g_{i l} \in C^{p}(\bar{V})$. Continue $u(z, t)$ by zero onto $[V \backslash \psi(U)] \times(0,2 T)$ and consider $Q_{\delta}^{2 T}=V_{\delta} \times(0,2 T)$, where

$$
V_{\delta}=\{z \in V: \sigma \geq-\delta\}
$$

Thus taking $V$ small enough the continued function $u \in L_{2}\left(Q_{\delta}^{2 T}\right)$ is a weak solution of the wave equation

$$
\square_{g} u=0, \text { in } Q_{\delta}^{2 T},
$$

satisfying

$$
\left.u\right|_{\Sigma_{\delta}^{2 T}}=\left.\partial_{\sigma} u\right|_{\Sigma_{\delta}^{2 T}}=0,
$$

where $\Sigma_{\delta}^{2 T}=\Gamma_{\delta} \times(0,2 T), \Gamma_{\delta}=\{z \in V: \sigma=-\delta\}$. Consider now curve $\tilde{z}(s)$, consisting of $z(s)$ and a part of a line, connecting points $z=(0, \ldots, 0,-\delta) \in \Gamma_{\delta}$ and 
$z=0$, then $\tilde{s}_{0} \leq s_{0}+C_{1} \delta, C_{1}>0$. Due to the compactness of $\Omega$, this curve $\tilde{z}(s)$ can be covered by a finite number of coordinate vicinities $U^{2}, \ldots, U^{K}, \mathrm{x} \in U^{K}$, such that

$$
\left\|g_{i l}\right\|_{C^{2}\left(\psi^{k}\left(U^{k}\right)\right)} \leq C_{2}, \quad g_{i l}(z) \xi^{i} \xi^{l} \geq C_{3}|\xi|^{2}
$$

where $\tilde{z} \in \overline{\psi^{k}\left(U^{k}\right)}$ and $C_{3}>0, k=2, \ldots, K$ and constants $C_{2}, C_{3}$ are constant for all vicinities. Thus the problem can be localized, i.e. we can assume that $\tilde{z}(s)$ belongs to some $\mathcal{D} \in \mathbb{R}^{n}, u \in L_{2}(\mathcal{D} \times(0,2 T))$ and

$$
\begin{gathered}
\square_{g} u=0 \text { in } \mathcal{D} \times(0,2 T), \\
\left.u\right|_{\Gamma_{0} \times(0,2 T)}=\left.\partial_{\sigma} u\right|_{\Gamma_{0} \times(0,2 T)}=0 ;
\end{gathered}
$$

here $\partial_{n+1} u=\frac{\partial u}{\partial \sigma}, 0 \ni \Gamma_{0} \subset\{(q, \sigma): \sigma=0\},(q=0, \sigma=0)=(0,0)$. Thus the proof is based on the continuation of initial data by zero along this curve from the point $\mathbf{y}$ to x. Consider now admissible Riemannian polyhedron $\mathcal{M}$.

Theorem 5.4 (Uniqueness theorem) Let $u \in L_{2}\left(Q^{2 T}\right), Q^{2 T}:=\mathcal{M} \times(0,2 T)$, be a weak solution to (5.3),(5.4), then $u=0$ a.e. in $K^{2 T}(\Gamma)$.

Proof Let us prove Theorem 5.4 in several steps:

(A) Let us fix $\mathbf{x} \in \mathcal{M}^{\text {int }} ; \mathcal{M}^{i n t}=\bigcup_{m=1}^{M} \Omega_{m}^{i n t}$, and $\varepsilon>0$. Due to (5.6) and the fact that $\Gamma$ is open, there exists $\mathbf{y} \in \Gamma, d_{\mathcal{M}}(\mathbf{x}, \mathbf{y}) \leq d_{\mathcal{M}}(\mathbf{x}, \Gamma)+\frac{\varepsilon}{4}$, moreover, $\mathbf{y} \in \Gamma \cap \gamma_{r}$, where $\gamma_{r}$ is some interface, such that $\gamma_{r} \subset \partial \Omega_{r}$, then due to results of Chapter 2, there exists a $C_{p}^{1}$ curve $z(s)$ of length $s_{0}$ :

$$
s_{0} \leq d_{\mathcal{M}}(\mathbf{x}, \Gamma)+\frac{\varepsilon}{2}
$$

connecting $\mathbf{x}$ and $\mathbf{y}: z(0)=\mathbf{y}, z\left(s_{0}\right)=\mathbf{x}$, such that the following conditions are satisfied:

(i) if $z(s)$ is a wedge point of the curve, i.e. $\dot{z}(s-0) \neq \dot{z}(s+0)$, then $z(s) \in \Omega_{m}^{\text {int }}$ for some $m$; 
(ii) curve $z(s), 0<s<s_{0}$ is transversal to all $W P^{j}, j \leq n-1$;

(iii) if $\mathrm{x}$ belongs to some interface or part of the boundary, then $z(s)$ is transversal to this interface and part of the boundary (the same is true for $y$ )(see also Lemma 2.4; see [25], [45]). Then the interval $\left[0, s_{0}\right]$ can be divided into a finite number of intervals $\left[0, s_{1}\right],\left[s_{1}, s_{2}\right], . .\left[s_{k}, s_{0}\right], 0<s_{1}<\ldots<s_{0}$, such that

$$
z(s) \in \Omega_{m(k)}^{i n t}
$$

with $k=0,1, \ldots, K, s_{K+1}=s_{0}$,

$$
z\left(s_{k}\right)=\gamma_{r(k)}, k=0,1, \ldots, K
$$

(B) Consider the second step of the proof:

\section{Proposition 5.2.1}

Consider the part of the curve $z(s) \in \Omega_{m(0)}$ with $s \in\left[0, s_{1}\right]$. The restriction of the solution $u$ of the problem (5.3),(5.4), onto $\Omega_{m(0)} \times(0,2 T)$ is a weak solution in $L_{2}\left(\Omega_{m(0)} \times(0,2 T)\right)$ to the Cauchy problem

$$
\left\{\begin{array}{l}
\square u=0 \text { in } \Omega_{m(0)} \times(0,2 T) \\
\left.u\right|_{\Gamma_{0} \times(0,2 T)}=\left.\partial_{\nu} u\right|_{\Gamma_{0} \times(0,2 T)}=0,
\end{array}\right.
$$

where $\Gamma_{0}=\Gamma \cap \gamma_{r(0)} \subset \partial \Omega_{m(0)}$, (we take the only part of $\Gamma$ that intersect with the only chamber, say, $\left.\Omega_{r(0)}\right), \mathrm{y} \in \Gamma_{0}$.

Proof of the proposition. Indeed, if $\psi \in C_{0}^{\infty}\left(\Omega_{m(0)} \times(0,2 T)\right), \Gamma_{0} \times(0,2 T)$, then continuing $\psi$ by zero onto $Q^{2 T}$ one can get function $\tilde{\psi} \in C^{0, \Delta}\left(Q^{2 T}, \Sigma^{2 T}\right)$. Thus

$$
\int_{\Omega_{m(0)} \times(0,2 T)} u \cdot \square \psi d x d t=\int_{Q^{2 T} \times(0,2 T)} u \cdot \square \tilde{\psi} d x d t=0
$$

for any $\psi \in C_{0}^{\infty}\left(\Omega_{m(0)} \times(0,2 T), \Gamma_{0} \times(0,2 T)\right)$, and thus $u$ is a weak solution of $(5.8)$ by the definition. 
(C) Using the result of Theorem 5.3 we have

$$
u=0 \text { a.e. } \operatorname{in}\left\{(\mathbf{z}, t): \mathbf{z} \in \Omega_{m(0)} ;|T-t| \leq T-d_{\Omega_{m(0)}}\left(\mathbf{z}, \Gamma_{0}\right)\right\},
$$

here $d_{\Omega_{m(0)}}\left(\mathbf{z}_{1}, \mathbf{z}_{2}\right)$ is a geodesic distance in metrics $g_{m(0)}$. Due to $(5.7)$ with $k=0$ $s_{1} \geq d_{\Omega_{m(0)}}\left(\mathbf{y}, z\left(s_{1}\right)\right)$, thus (5.9) implies that for any $\delta>0$ there exists a vicinity $U_{1} \ni z\left(s_{1}\right)$ such that

$$
u=0 \text { a.e. in }\left\{(\mathbf{z}, t): \mathbf{z} \in U_{1} \cap \Omega_{m(0)} ;|T-t| \leq T-s_{1}-\delta\right\} .
$$

(D) Consider the second part of the curve $z(s)$ for $s \in\left[s_{1}, s_{2}\right]$ belonging to $\Omega_{m(1)}$. We need to show that (5.10) implies that $u$, restricted onto $\Omega_{m(1)} \times\left(s_{1}+\delta, 2 T-s_{1}-\delta\right)$ is a solution to the Cauchy problem

$$
\left\{\begin{array}{l}
\square u=0 \text { in } \Omega_{m(1)} \times\left(s_{1}+\delta, 2 T-s_{1}-\delta\right) ; \\
u=\left.\partial_{\nu} u\right|_{\Gamma_{1} \times\left(s_{1}+\delta, 2 T-s_{1}-\delta\right)}=0,
\end{array}\right.
$$

for some open set $\Gamma_{1} \subset \partial \Omega_{m(1)}$ :

$$
\Gamma_{1} \subset U_{1} \cap \gamma_{(m(1))}, z\left(s_{1}\right) \in \Gamma_{1} .
$$

(E) Denote now $\Omega_{-}:=\Omega_{m(0)}, \Omega_{+}:=\Omega_{m(1)}$, and consider another notations. Consider $\mathcal{M}=\Omega_{-} \cup \Omega_{+}$to be our manifold, $\gamma$ is a common interface between $\Omega_{ \pm}$as we considered them for Gaussian beams. We use the following notations:

$$
\Gamma_{0} \subset \partial \mathcal{M} \cap \partial \Omega_{-}, \quad \Gamma_{0} \neq \emptyset
$$

is an open subset of the boundary,

$$
\Sigma_{0}^{2 T}=\Gamma_{0} \times(0,2 T)
$$

is a boundary lane,

$$
Q^{2 T}=\mathcal{M} \times(0,2 T)
$$

the cone of influence is

$$
K^{2 T}\left(\Gamma_{0}\right)=\left\{(\mathrm{x}, t) \in Q^{2 T}:|T-t| \leq T-d_{\Omega}\left(\mathbf{x}, \Gamma_{0}\right)\right\}
$$

where $d_{\mathcal{M}}\left(\mathbf{x}, \Gamma_{0}\right)=\inf _{\mathbf{y} \in \Gamma_{0}} d_{\mathcal{M}}(\mathbf{x}, \mathbf{y})$. 
Notation 5.2 Here we used the following notations:

$$
\begin{gathered}
C_{0}^{\infty}(R, B)=\left\{f \in C^{\infty}(\bar{R}): \operatorname{supp} f \cap(\partial R \backslash B)=\emptyset\right\}, \\
C^{0, \Delta_{g}}\left(W \times\left(\tau_{1}, \tau_{2}\right)\right)=C\left(\left(\tau_{1}, \tau_{2}\right), \mathcal{D}\left(\Delta_{g}\right)\right) \cap C^{2}\left(\left(\tau_{1}, \tau_{2}\right), L_{2}(W)\right), \\
\mathcal{D}\left(\Delta_{g}\right)=\left\{\xi \in H^{1}(W): \Delta_{g} \xi \in L_{2}(W)\right\} .
\end{gathered}
$$

Lemma 5.1 (Lemma 2 from [45]) Consider $\gamma \cap \Gamma_{0}=\emptyset, \gamma \cap W P^{j}=\emptyset, j \leq n-2$. Then if $\psi \in C_{0}^{\infty}\left(\Omega_{-} \times\left(\tau_{1}, \tau_{2}\right), \gamma \times\left(\tau_{1}, \tau_{2}\right)\right),\left(\tau_{1}, \tau_{2}\right) \subset(0,2 T)$, there exists a function $\widetilde{\psi} \in C_{0}^{0, \Delta_{g}}\left(Q^{2 T}, \Sigma_{0}^{2 T}\right)$ such that

$$
\left.\widetilde{\psi}\right|_{\Omega_{-} \times\left(\tau_{1}, \tau_{2}\right)}=\psi
$$

Moreover, if $\gamma \Subset \widetilde{U}$, where $\widetilde{U}$ is some neighborhood, then $\tilde{\psi}$ can be chosen such that

$$
\operatorname{supp}(\widetilde{\psi}-\psi) \subset \widetilde{U} \times \dot{\left(\tau_{1}, \tau_{2}\right)}
$$

Proof of Lemma 5.1. We consider $\gamma \subset \Omega_{-} \cup \Omega_{+}$, such that $\gamma \Subset U$, where $U$ is a coordinate neighborhood such that $\phi(U)=W$ is a domain in $\mathbb{R}^{n}$, where

$$
\phi\left(U \cap \Omega_{-}\right)=W_{-}, \phi\left(U \cap \Omega_{+}\right)=W_{+}, \phi(\gamma) \Subset W_{0}
$$

where

$$
\begin{gathered}
W_{+}=\left\{\mathbf{q} \in W: q^{n}=\sigma \geq 0\right\}, W_{0}=\left\{\mathbf{q} \in W: q^{n}=\sigma=0\right\}, 0 \in W, \phi(\mathbf{y})=0, \\
W_{-}=\left\{\mathbf{q} \in W: q^{n}=\sigma \leq 0\right\} .
\end{gathered}
$$

Besides that we can assume that $\psi \in C_{0}^{\infty}\left(\Omega_{-} \times\left(\tau_{1}, \tau_{2}\right)\right)$ is such that

$$
\operatorname{supp} \psi \subset\left\{U \cap \Omega_{-}\right\} \times\left(\tau_{1}, \tau_{2}\right)
$$

In coordinates (5.14) we have

$$
\psi \in C_{0}^{\varsigma}\left(W_{-} \times\left(\tau_{1}, \tau_{2}\right)\right), g_{ \pm}^{i l} \in C^{\varsigma-1}\left(W_{ \pm}\right), i, l=1, \ldots, n
$$


Consider function $\widetilde{\psi}$ on $W \times\left(\tau_{1}, \tau_{2}\right)$ of the following form

$$
\widetilde{\psi}(\mathbf{q}, t)=\left\{\begin{array}{l}
\psi_{-}(\mathbf{q}, t):=\psi(\mathbf{q}, t), \sigma \leq 0 \\
\psi_{+}(\mathbf{q}, t), \sigma \geq 0 \\
\left.\psi_{-}\right|_{\sigma=0}=\left.\psi_{+}\right|_{\sigma=0},\left.\sqrt{g^{-}} \partial_{\sigma} \psi_{-}\right|_{\sigma=0}=\left.\sqrt{g^{+}} \partial_{\sigma} \psi_{+}\right|_{\sigma=0}
\end{array}\right.
$$

where

$$
\psi_{+}\left(\mathbf{q}^{\alpha},-\sigma, t\right)=\left[\kappa_{1}\left(\mathbf{q}^{\alpha}\right) \psi\left(\mathbf{q}^{\alpha}, \sigma, t\right)+\kappa_{2}\left(\mathbf{q}^{\alpha}\right) \psi\left(\mathbf{q}^{\alpha}, 2 \sigma, t\right)\right] \chi(\sigma)
$$

where $q^{\alpha}=\left(q^{1}, . ., q^{n-1}\right)$, and $\chi$ is a cut-off function such that $\chi(0)=1$, and $\chi^{\prime}(0)=0$, and the interface continuity conditions imply that

$$
\begin{gathered}
\kappa_{1}\left(\mathbf{q}^{\alpha}\right)+\kappa_{2}\left(\mathbf{q}^{\alpha}\right)=1 \\
\left.\frac{\sqrt{g_{+}}}{\sqrt{g_{-}}}\right|_{\sigma=0}-1=\kappa_{2}\left(\mathbf{q}^{\alpha}\right) .
\end{gathered}
$$

Thus

$$
\text { supp } \widetilde{\psi} \Subset W \times\left(\tau_{1}, \tau_{2}\right) \text { and } \widetilde{\psi} \in C_{0}^{0, \Delta_{g}}\left(W \times\left(\tau_{1}, \tau_{2}\right)\right)
$$

If $\widetilde{U} \subset U$ is some neighborhood of $\phi(\gamma)$, then using a special choice of $\chi$ one can have

$$
\operatorname{supp}(\widetilde{\psi}-\psi) \subset \widetilde{U} \times\left(\tau_{1}, \tau_{2}\right) \text {. }
$$

Finally, continuing $\widetilde{\psi}$ by zero outside $U \times\left(\tau_{1}, \tau_{2}\right)$, we get the function (we still denote it by $\widetilde{\psi}) \widetilde{\psi}$ such that $\widetilde{\psi} \in C^{0, \Delta_{g}}\left(Q^{2 T}, \Sigma_{0}^{2 T}\right)$ :

$$
\left.\widetilde{\psi}\right|_{\Omega_{-} \times\left(\tau_{1}, \tau_{2}\right)}=\psi
$$

Thus we have proven the lemma.

(F) We can show that lemma implies the following result:

Lemma 5.2 (Corollary) If $u$ is a weak solution to (5.3),(5.4), such that $u=0$ a.e. in $\left\{\widetilde{U} \cap \Omega_{-}\right\} \times\left(\tau_{1}, \tau_{2}\right)$, where $\widetilde{U}$ is some neighborhood of $y$, such that

$$
y \in \gamma \cap \widetilde{U}
$$


then there exists an open set $\gamma_{0} \subset \gamma$, such that function $u$, restricted onto $\Omega_{+} \times\left(\tau_{1}, \tau_{2}\right)$ is a weak solution to the problem

$$
\left\{\begin{array}{l}
\square_{g} u=0, \text { in } \Omega_{+} \times\left(\tau_{1}, \tau_{2}\right), \\
\left.u\right|_{\gamma_{0} \times\left(\tau_{1}, \tau_{2}\right)}=\left.\partial_{\nu} u\right|_{\gamma_{0} \times\left(\tau_{1}, \tau_{2}\right)}=0 .
\end{array}\right.
$$

Proof We should notice, that without a loss of generality, we can assume that $u \subset$ $\Omega_{-} \cup \Omega_{+}$. Let $\gamma_{0} \subset \gamma$ be an open set, such that $\mathrm{y} \in \gamma_{0} \Subset U$; the existence of $\gamma_{0}$ is guaranteed by the choice of $\mathbf{y}$. If $\tilde{\psi} \in C_{0}^{\infty}\left(\Omega_{+} \times\left(\tau_{1}, \tau_{2}\right)\right)$, then due to Lemma 5.1, there exists its continuation $\tilde{\psi} \in C^{0, \Delta_{g}}\left(Q^{2 T}, \Sigma_{0}^{2 T}\right)$ such that

$$
\operatorname{supp}(\tilde{\psi}-\psi) \subset \tilde{U} \times\left(\tau_{1}, \tau_{2}\right)
$$

As $u$ is the weak solution to $(5.3),(5.4)$, and $u=0$ a.e. in $\left\{\tilde{U} \cap \Omega_{-}\right\} \times\left(\tau_{1}, \tau_{2}\right)$, then

$$
\int_{Q^{2 T}} u \cdot \square_{ \pm} \widetilde{\psi} d x d t=\int_{\Omega_{+} \times\left(\tau_{1}, \tau_{2}\right)} u \cdot \square_{ \pm} \psi d x d t=0
$$

for all $\psi \in C_{0}^{\infty}\left(\Omega_{+} \times\left(\tau_{1}, \tau_{2}\right), \gamma_{0} \times\left(\tau_{1}, \tau_{2}\right)\right)$.

Next, we return to our considerations of the curve $z(s)$, considering again $\Omega_{m(1)}$ to be a smooth manifold with piece-wise smooth boundary and assuming that

$$
s_{2}-s_{1} \geq d_{\Omega_{m(1)}}\left(z\left(s_{1}\right), z\left(s_{2}\right)\right)
$$

we get that there exists a vicinity $U_{2} \ni z\left(s_{2}\right)$ such that

$$
u=0 \text { a.e. in }\left\{(\mathbf{z}, t): \mathbf{z} \in U_{2} \cap \Omega_{m(1)} ;|T-t| \leq T-s_{2}-2 \delta\right\}
$$

(G) We continue the process $K+1$ times and show that there exists a vicinity $U_{x} \ni \mathbf{x}$ such that

$$
u=0 \text { a.e. } \operatorname{as}\left\{(\mathbf{z}, t): \mathbf{z} \in U_{x} ;|T-t| \leq T-s_{0}-(k+1) \delta\right\},
$$


where we assume that $\mathbf{x} \in \mathcal{M}^{\text {int }}$. Then taking $\delta=[2(K+1)]^{-1} \varepsilon$ one gets

$$
u=0 \text { a.e. } \operatorname{as}\left\{(\mathrm{z}, t): \mathbf{z} \in U_{x} ;|T-t| \leq T-d_{\mathcal{M}}(\mathbf{x}, \Gamma)-\varepsilon\right\} .
$$

(H) As $d_{\Omega}(\mathrm{x}, \mathrm{y})$ is Lip continuous with respect to $(\mathrm{x}, \mathrm{y})$, then last relation in Theorem (5.4) follows from (5.20) as $\varepsilon>0$ is arbitrarily chosen and as $\overline{\Omega^{i n t}}=\Omega$. Thus we have proven Theorem 5.4.

That was a generalization of Theorem 4.2. 

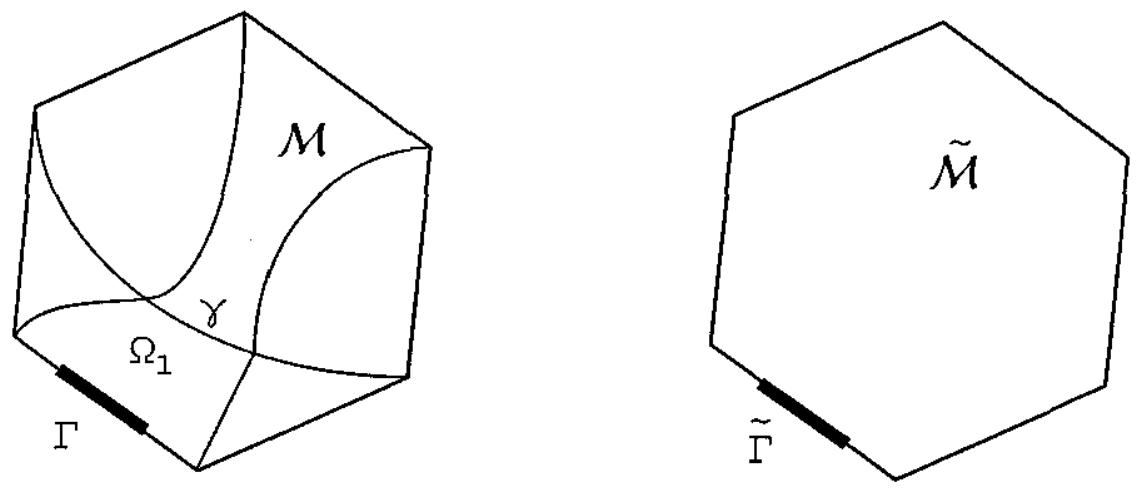

Figure 5.1: Two admissible Riemannian polyhedra with equal BSD

\subsection{Uniqueness inverse problem}

Now we are ready to show that boundary spectral data given on an open part of polyhedron boundary $\Gamma$ determine the polyhedron uniquely. To this end consider two admissible Riemannian polyhedra $\mathcal{M}$ and $\widetilde{\mathcal{M}}$, such that

$$
\operatorname{BSD}\left(\Gamma, \Delta_{g}\right)=\operatorname{BSD}\left(\widetilde{\Gamma} \subset \partial \widetilde{\mathcal{M}}, \widetilde{\Delta}_{\tilde{g}}\right)
$$

where $\Gamma \subset \partial \mathcal{M}$, and $\widetilde{\Gamma} \subset \partial \widetilde{\mathcal{M}}$ are open subsets. In other words, see (5.2), $\Gamma$ is diffeomorphic to $\widetilde{\Gamma}$,

$$
\begin{aligned}
\lambda_{k} & =\tilde{\lambda}_{k}, \\
\left.\varphi_{k}\right|_{\Gamma} & =\left.\tilde{\varphi}_{k}\right|_{\tilde{\Gamma}} .
\end{aligned}
$$

We assume that $\mathcal{M}$ is given and we use $\widetilde{\mathcal{M}}$ to show that it is isometric to $\mathcal{M}$. We start from the open subset $\Gamma$ which is assumed (without a loss of generality) to have nonzero intersection with the only chamber boundary, say, $\partial \Omega_{1}$, see Figure 5.1. We find the same picture on $\widetilde{\mathcal{M}}$ as well.

There exist $\tau_{\Gamma}, \widetilde{\tau}_{\widetilde{\Gamma}}$ (critical value function with respect to points of $\Gamma, \widetilde{\Gamma}$, see (4.33)), corresponding to $\Gamma, \widetilde{\Gamma}$ on each polyhedra. We can find them, as both polyhedra 

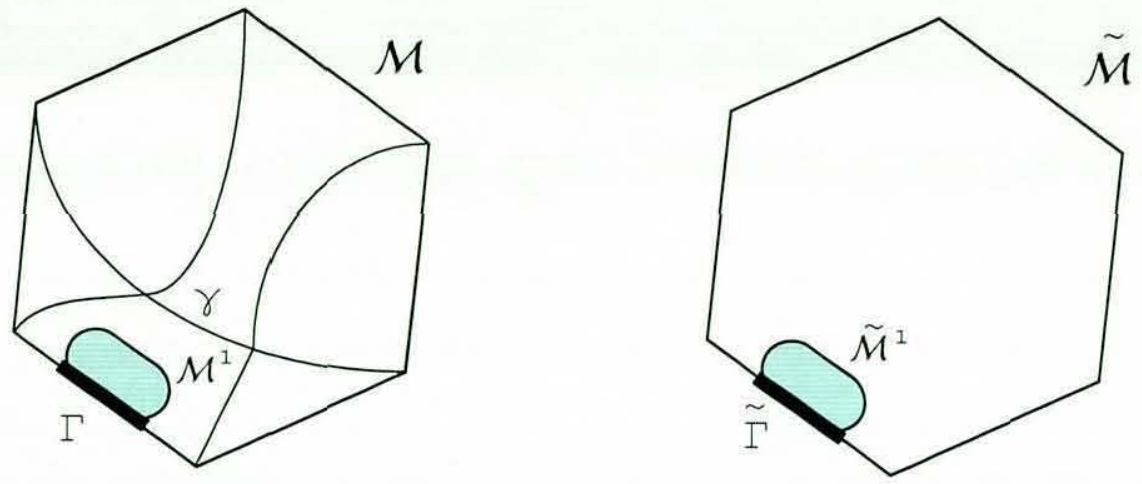

Figure 5.2: First subset identical on both polyhedra

are at our disposal. Consider some smooth coordinates $\left\{\mathbf{z}^{\alpha}\right\} \in \Gamma$, as $\Gamma$ is isometric to $\widetilde{\Gamma}$, we can choose equal smooth coordinates $\left\{\tilde{\mathbf{z}}^{\alpha}\right\} \in \widetilde{\Gamma}$. Then there exist regular boundary normal coordinates $(\mathbf{z}, s)$ and $(\tilde{\mathbf{z}}, \tilde{s})$, see Notation 2.12 , starting from $\Gamma$ (and $\widetilde{\Gamma}$ correspondingly) at least for $s<\tau_{0}$. Here

$$
\tau_{0}=\min \left(\tau_{\Gamma}, \tilde{\tau}_{\widetilde{\Gamma}}\right),
$$

is the minimal value between two.

Consider the first subset $\mathcal{M}^{1}=\mathcal{M}_{\Gamma} \subset \mathcal{M}$, see (4.35), see Figure 5.2. We are in the situation described for the smooth manifold in [38], (see also Chapter 4, subsections 4.2.1 and 4.2.2). We continue eigenfunctions $\varphi_{k}, \widetilde{\varphi}_{k}$ inside $\mathcal{M}_{\Gamma}, \widetilde{\mathcal{M}}_{\widetilde{\Gamma}}$, where $\tau<\tau_{0}$, using Results 4.2.1.1 and 4.2.1.2. Thus $\widetilde{\mathcal{M}}_{\widetilde{\Gamma}}$ should be identical to $\mathcal{M}_{\Gamma}$. Thus, $\mathcal{M}^{1}$ is isometric to $\widetilde{\mathcal{M}^{1}}$.

We remove $\Gamma, \widetilde{\Gamma}$ now, and consider $\mathcal{M}_{\Gamma} \backslash \Gamma$ and $\widetilde{\mathcal{M}}_{\Gamma} \backslash \widetilde{\Gamma}$, the open subsets (domains) inside chambers $\Omega_{1}$ and $\widetilde{\Omega}_{1}$. Next we choose any two subsets with smooth boundaries $\mathcal{D} \subset \mathcal{M}_{\Gamma}$ and $\widetilde{\mathcal{D}} \subset \widetilde{\mathcal{M}}_{\tilde{\Gamma}}$, see Figure 5.3. There exists $\delta$ for both polyhedra (not necessarily small) such that $\mathcal{D}_{\delta} \subset \Omega_{1}$ and $\widetilde{\mathcal{D}}_{\delta} \subset \widetilde{\Omega}_{1}$ and, besides that, the boundary normal coordinates $(2.12)$, based on $\partial \mathcal{D}(\partial \widetilde{\mathcal{D}})$, are regular. Using procedures from [38] (and also their descriptions in Chapter 4.2.3, Subsection 4.2.1, replacing $\Gamma$ by $\partial \mathcal{D}$ 


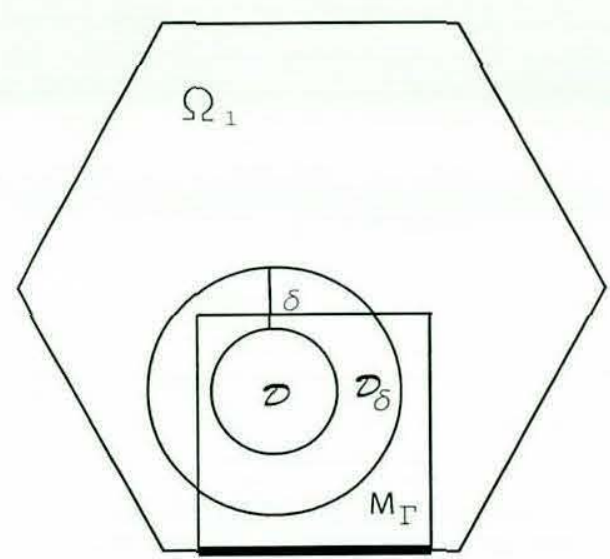

$\Gamma$

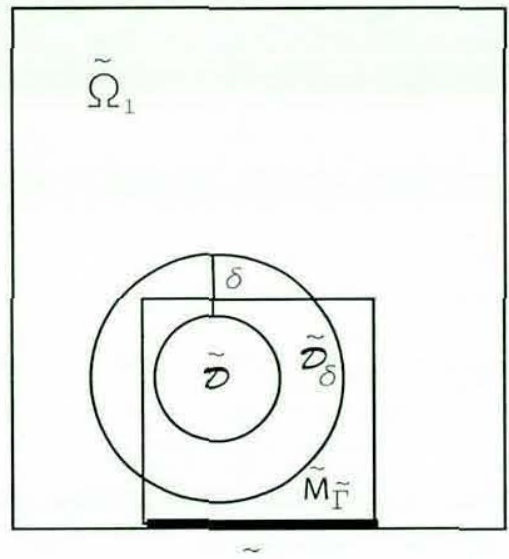

$\Gamma$

Figure 5.3: First chamber on both manifolds. We choose open domains inside first chambers.

for $\mathcal{M}$ and by $\partial \widetilde{\mathcal{D}}$ for $\widetilde{\mathcal{M}}$ and applying Lemma 4.3), we can obtain results, similar to Results 4.2.1.1 and 4.2.1.2, we reconstruct eigenfunctions $\left.\varphi_{k}\right|_{\mathcal{D}_{\delta}}=\left.\widetilde{\varphi}_{k}\right|_{\widetilde{\mathcal{D}}_{\delta}}\left(\varphi_{k}, \widetilde{\varphi}_{k}\right.$ inside $\left.\mathcal{D}_{\delta}, \widetilde{\mathcal{D}}_{\delta},\right)$ as $\partial \mathcal{D}_{\delta} \subset \mathcal{M}_{\Gamma}$, and $\partial \widetilde{\mathcal{D}}_{\delta} \subset \widetilde{\mathcal{M}}_{\tilde{\Gamma}}$. Then $\mathcal{D}_{\delta}$ is isometric to $\widetilde{\mathcal{D}}_{\delta}$.

Moreover, the subset $\mathcal{M}_{\Gamma} \cup \mathcal{D}_{\delta}$ is isometric to $\widetilde{\mathcal{M}}_{\widetilde{\Gamma}} \cup \widetilde{\mathcal{D}}_{\delta}$, as $\left.\varphi_{k}\right|_{\mathcal{M}_{\Gamma} \cup \mathcal{D}_{\delta}}$ are equal to corresponding $\left.\widetilde{\varphi}_{k}\right|_{\widetilde{\mathcal{M}}_{\tilde{\Gamma}} \cup \widetilde{\mathcal{D}}_{\delta}}$. The procedure of eigenfunctions continuation from $\mathcal{M}_{\Gamma}$ to $\mathcal{D}_{\delta}$ is described section 4.4 of [38] (also in the Section 4.2.3). As we are now in dealing with smooth parts of our polyhedra, we can apply this procedure of previous chapter.

Consider $\mathcal{M}_{\Gamma} \cup \mathcal{D}_{\delta}$ as a union of intersecting sets, then we "glue" by the procedure, introduced in [38] and described in Subsection 4.2.3.

We identify points $\mathrm{x} \in \mathcal{M}_{\Gamma}$ and $\hat{\mathrm{x}} \in \mathcal{D}_{\delta}$ such that $\varphi_{k}(\mathrm{x})=\varphi_{k}(\hat{\mathrm{x}})$, for any $k=$ $1,2, \ldots$, see Chapter 2 for details. In this case points $\mathbf{x}$ and $\hat{\mathbf{x}}$ correspond to the same point on $\mathcal{M}$. We can choose another subsets $\mathcal{D}_{\delta}^{\prime}$ and $\widetilde{\mathcal{D}}_{\delta}^{\prime}$ and repeat the procedure on their reconstruction. Thus we also identify points $\mathrm{x} \in \mathcal{D}_{\delta}$ with points of similarly constructed $\mathrm{x}^{\prime} \in \mathcal{D}_{\delta}^{\prime}$, and with $\hat{\mathrm{x}} \in \mathcal{M}_{\Gamma}$ if the eigenfunctions of these points coincide. 

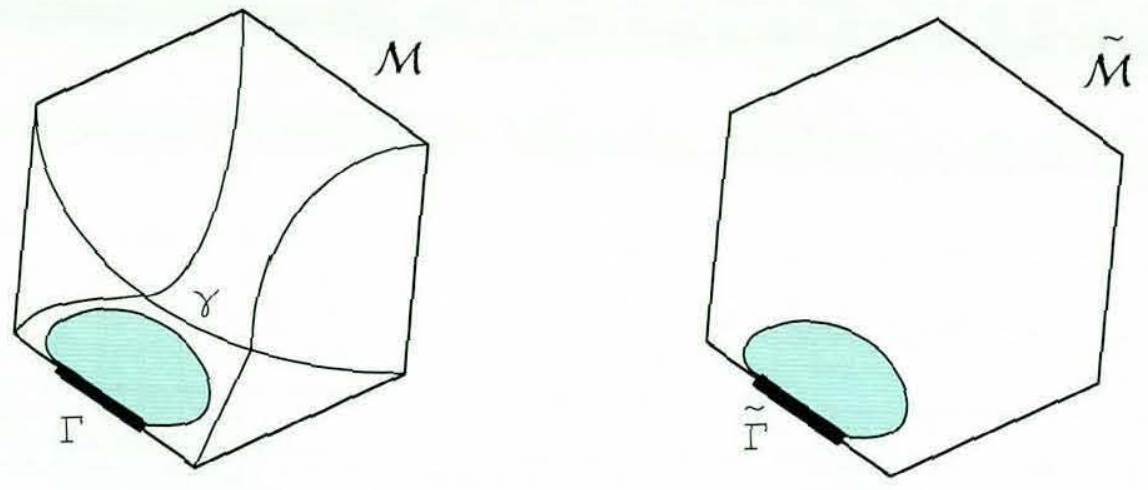

Figure 5.4: Increasing subsets identical on both polyhedra

We apply the same procedure on $\tilde{\mathcal{M}}$ and as the eigenfunctions $\varphi_{k}$ and $\widetilde{\varphi}_{k}$ are equal on $\mathcal{D}_{\delta}$ and $\widetilde{\mathcal{D}}_{\delta} ; \mathcal{D}_{\delta}^{\prime}$ and $\widetilde{\mathcal{D}}_{\delta}^{\prime} ; \mathcal{M}_{\Gamma}$ and $\widetilde{\mathcal{M}}_{\tilde{\Gamma}}$, we form the isometric subsets $\mathcal{M}^{2}$ and $\widetilde{\mathcal{M}}^{2}$ as a maximal union of equal points obtained for all $\mathcal{D} \subset \mathcal{M}_{\Gamma}$ and $\widetilde{\mathcal{D}} \subset \widetilde{\mathcal{M}}_{\widetilde{\Gamma}}$, such that the eigenfunctions are equal on $\mathcal{D}$ and $\widetilde{\mathcal{D}}$.

As we used the procedure described in Section 4.2.3, which allows to recalculate eigenfunctions uniquely on each step, and as we identified all equal points, thus $\mathcal{M}^{2}$, constructed subset of $\Omega_{1} \subset \mathcal{M}$ as isometric to $\widetilde{\mathcal{M}}^{2}$, subset of $\widetilde{\Omega}_{1} \subset \widetilde{\mathcal{M}}$, see Figure 5.4.

We continue increasing our subsets step by step. Let us denote by $\Omega_{1}^{0}$ the maximal subset inside $\Omega_{1}$ and $\widetilde{\Omega}_{1}^{0}$ to be the maximal $\widetilde{\Omega}_{1}$, such that $\Omega_{1}^{0}$ is isometric to $\widetilde{\Omega}_{1}^{0}$. 

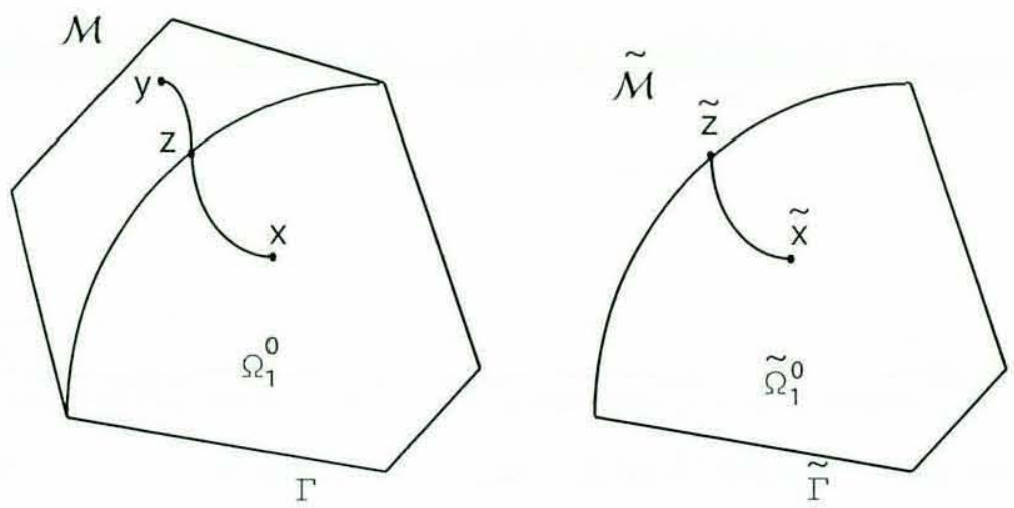

Figure 5.5: We assumed that the maximal subset is "less" than the first chamber.

\subsection{Meeting the interface}

In this section we prove, that the first chambers $\Omega_{1}$ and $\widetilde{\Omega}_{1}$ are isometric, i.e. that maximal subsets $\Omega_{1}^{0}$ and $\widetilde{\Omega}_{1}^{0}$ coincide with $\Omega_{1}$ and $\widetilde{\Omega}_{1}$.

Assume that $\Omega_{1} \backslash \Omega_{1}^{0} \neq \emptyset$. Consider points

$$
\mathrm{y} \in \Omega_{1} \backslash \Omega_{1}^{0}
$$

and $\mathrm{x} \in \Omega_{1}^{0}$. Let also $\tilde{\mathrm{x}}$ be the point corresponding to $\mathrm{x}$ in $\widetilde{\Omega}_{1}^{0}$ (we can find it as we have already shown, that $\Omega_{1}^{0}$ is isometric to $\widetilde{\Omega}_{1}^{0}$.)

Choose now some curve $\eta$ in $\Omega_{1}$ joining points $\mathbf{y}$ and $\mathbf{x}$. Let $\mathrm{z}$ be the first point of $\eta$ from $\mathrm{x}$ which is outside $\Omega_{1}^{0}$. Then support of the part of the curve $\eta(\mathrm{x}, \mathrm{z})$ should belong to $\Omega_{1}^{0}$, (otherwise, $\mathrm{z}$ is not the first point outside), see Figure 5.5. Then the corresponding to it part of the curve $\widetilde{\eta}(\tilde{\mathbf{x}}, \tilde{\mathbf{z}})$ belongs to $\widetilde{\Omega}_{1}^{0}$. Then necessarily

$$
\tilde{\mathbf{z}} \in \partial \widetilde{\Omega}_{1} .
$$



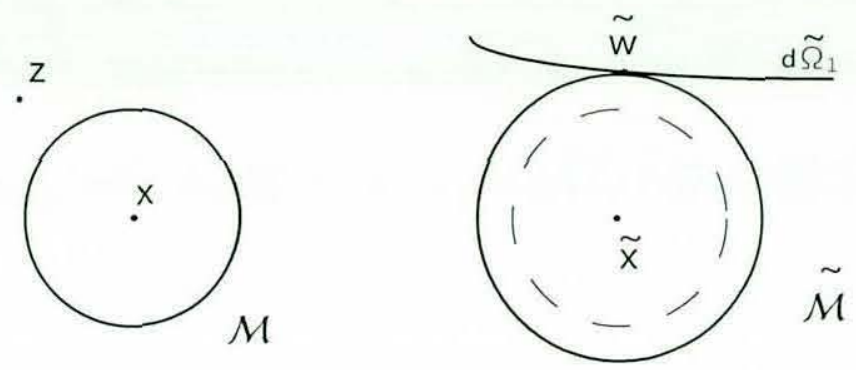

Figure 5.6: Interface point on the second polyhedron.

Indeed, otherwise, $\Omega_{1}^{0}$ and $\widetilde{\Omega}_{1}^{0}$ are not maximal, we are able to add two ball $B, \tilde{B}$ with centers at $\mathbf{z}$ and $\tilde{\mathbf{z}}$, and continue procedures of the previous subsection.

Next we construct a ball with center in $\mathrm{x}$ (we can choose $\mathrm{x}$ to be close to $\mathrm{z}$, if we need) see Figure 5.6. With some radius $r$, such that $r \leq d(\mathrm{x}, \mathrm{z})$, the ball in $\widetilde{\mathcal{M}}$ will touch $\partial \widetilde{\Omega}_{1}$ for the first time. Let us call that point $\tilde{w} \in \widetilde{\mathcal{M}}$. We should mention, that for $r \leq d(\mathbf{x}, \mathbf{z})$, the constructed ball in $\mathcal{M}$ belongs to $\Omega_{1}^{0}$. Consider the cases of $\tilde{w} \in \partial \widetilde{\Omega}_{1}$ position.

1. Let $\tilde{w}$ be the point of the interface boundary, i.e. $\tilde{w} \in \tilde{\gamma}$, see Figure 5.6. Then the interface $\tilde{\gamma}$ touches the ball at $\tilde{w}$. The Gaussian beam, normal to the ball $\tilde{B}$ of radius $\frac{r}{2}$ reflects with close to $\frac{\pi}{2}$ angle on $\widetilde{\mathcal{M}}$ ( see reflection angle formula (3.63)). But that would not be the case for ball $B \subset \mathcal{M}$ (Consider formulae (3.72), or (3.73), we compare the reflection and transmission coefficients 3.3 for the case with interface and without interface. One can see, that in our case, when the Gaussian beam starting from the ball $B$, it does not meet the interface in time $\frac{r}{2}$, thus the reflection coefficient $\mathcal{R}=0$. Oppositely, the Gaussian beam on $\widetilde{\mathcal{M}}$, starting from $\tilde{B}$, will reflect to $\tilde{B}$ with non-zero $\mathcal{R}$, compare Parts (C), (B) with (A) on Figure 5.8.) Comparing $\varphi_{k}$ and $\widetilde{\varphi}_{k}$ (that are found from the information obtained from Gaussian beams, see Subsection 4.2.1 for details), we will understand, that there is a reflection in one case and there is no reflection 

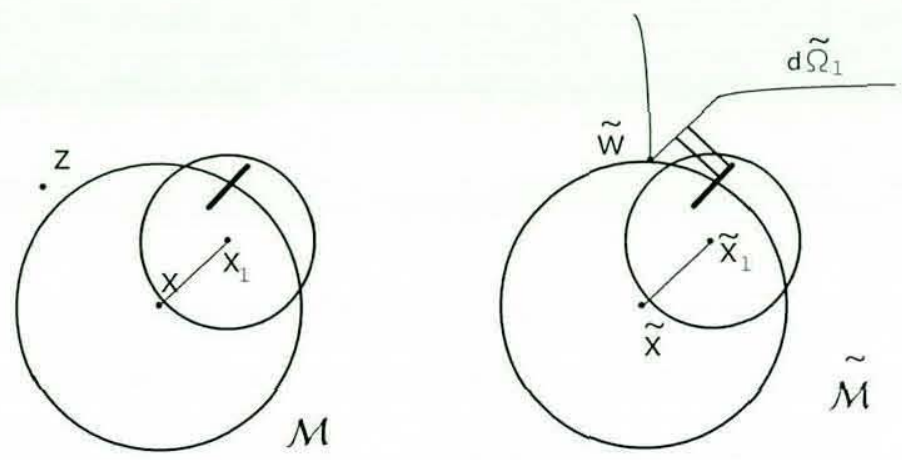

Figure 5.7: Wedge point on the second polyhedron.

in the second case. But we have previously shown that $\varphi_{k}$ and $\widetilde{\varphi}_{k}$ are equal on $\Omega_{1}^{0}$ and $\widetilde{\Omega}_{1}^{0}$.

2. Point $\tilde{w} \in W P^{j}, j \leq n-2$, can not be a wedge point, because we do not have an atrificial interfaces on $\mathcal{M}$, see Definition 2.21. Picture of the type like Figure 5.7 can not appear on an admissible Riemannian polyhedron.

3. Let $\tilde{w} \in \partial \widetilde{M}$, be a point on the global boundary of the polyhedron. This case is similar to the first one, the only difference is that the reflection coefficient $\mathcal{R}=1$ and $\mathcal{T}=0$ on $\widetilde{\mathcal{M}}$, see schematic pictures (A), (B), (C) and (D) on Figure 5.8 .

Thus we came to the contradiction with assumption that $\mathrm{y} \in \Omega_{1} \backslash \Omega_{1}^{0}$ and thus $\Omega_{1}$ is isometric to $\widetilde{\Omega}_{1}$ in the inner metric. 


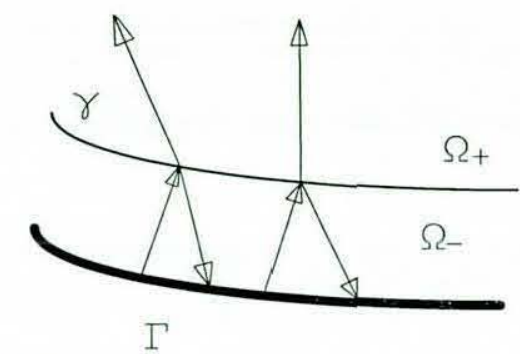

(A)

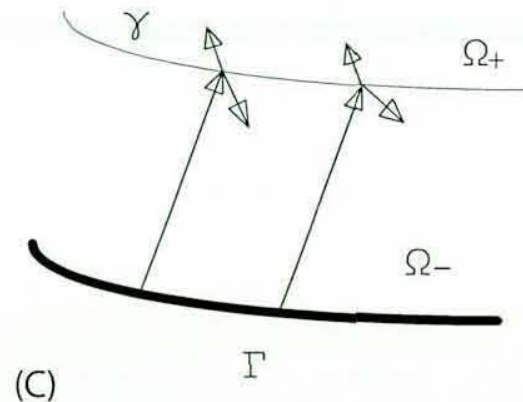

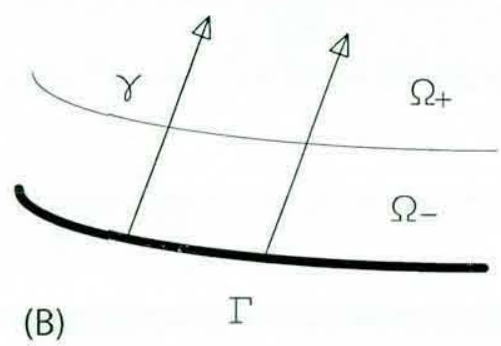

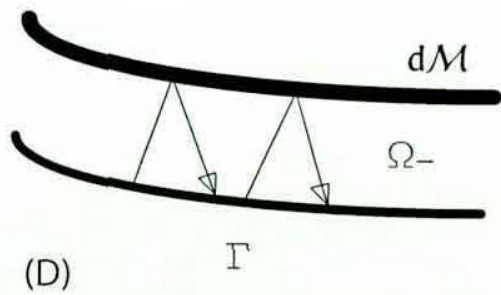

Figure 5.8: Various types of Gaussian beams reflections. 


\subsection{Crossing the interface}

We want to choose the equal parts the interfaces boundaries of $\Omega_{1}, \widetilde{\Omega}_{1}$, say $\gamma$ and $\tilde{\gamma}$. As we have obtained above, chamber $\Omega_{1}$ is isometric to $\widetilde{\Omega}_{1}$, metric tensors are equal, and eigenfunctions $\left.\varphi_{k}\right|_{\Omega_{1}}$ are equal to $\left.\tilde{\varphi}_{k}\right|_{\tilde{\Omega}_{1}}$. We also know, that inside each chamber the eigenfunctions identify points, see [59], also see Lemma 2.6. We choose Cauchy sequences of "equal" points.

Notation 5.3 (Equal points) We call two points $\mathrm{x} \in \mathcal{M}$ and $\tilde{\mathrm{x}} \in \widetilde{\mathcal{M}}$ equal if the set of eigenfunctions values at these points are equal, i.e. $\varphi_{k}(\mathrm{x})=\widetilde{\varphi}_{k}(\tilde{\mathrm{x}}), k=1,2, \ldots$. In the case when such points belong to the same polyhedron, we identify these points and say, that this is one point.

Define by $q \in \partial \Omega_{1}$ the limit point of a Cauchy sequence $\left\{x_{i}\right\} \in \Omega_{1}^{\text {int }}$, such that these sequence does not have a limit in $\Omega_{1}^{i n t}$. The Cauchy sequence $\left\{\tilde{x}_{i}\right\}$ of equal points has a limit point $\tilde{q} \in \partial \widetilde{\Omega}_{1}$. We can consider the distance between the points of two Cauchy sequences on the same polyhedron. Let $\left\{x_{i}\right\}$ and $\left\{x_{i}^{\prime}\right\}$ be two Cauchy sequences, then if $\lim _{m \rightarrow 0} d_{\Omega_{1}^{i n t}}\left(x_{m}, x_{m}^{\prime}\right)=0$, then these two sequences are equivalent, and they have the same limit point $q \in \partial \Omega_{1}$.

Thus now we consider the Cauchy sequences on $\mathcal{M}$, such that they have their limit points on the open part $\gamma$ of $\partial \Omega_{1}$. We can also consider "equal" Cauchy sequences on $\widetilde{\mathcal{M}}$. These sequences are also Cauchy sequences which define $\tilde{\gamma}$ isometric to $\gamma$. (Measuring distances between points of these Cauchy sequences, we can see that the limit points will belong to some open part $\tilde{\gamma} \subset \widetilde{\Omega}_{1}$, isometric to $\gamma$.) Each point $\tilde{q}$ will be equal to $q$, (see Remark 2.5.3).

The parts of the interface boundaries $\gamma$ and $\tilde{\gamma}$ equal for both polyhedra. 

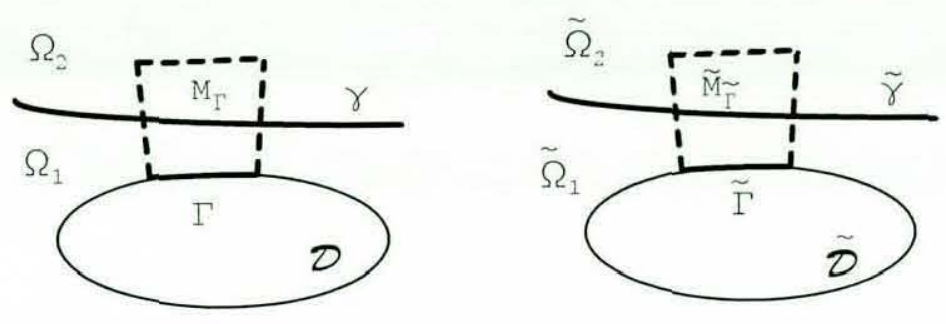

Figure 5.9: Crossing the interface on both polyhedra.

Consider now equal parts of the interfaces $\gamma$ and $\tilde{\gamma}$ obtained above. Let us construct two open subsets $\mathcal{D} \subset \Omega_{1}$ and $\widetilde{\mathcal{D}} \subset \widetilde{\Omega}_{1}$ with smooth boundaries $\partial \mathcal{D}$ and $\partial \widetilde{\mathcal{D}}$. We can choose them to be close to $\gamma$ and $\tilde{\gamma}$ correspondingly. Let us choose the "upper" parts of their boundaries $\Gamma$ and $\tilde{\Gamma}$, that are parallel and close to $\gamma$ and $\tilde{\gamma}$, see Figure 5.9.

Then there exists some $\tau_{0}=\min \left\{\tau_{\Gamma}, \tau_{\tilde{\Gamma}}\right\}$ (see (4.33)), such that the beam of extremal rays gives us regular coordinates $\mathbf{z}^{\alpha} \in \Gamma, \tilde{\mathbf{z}}^{\alpha} \in \tilde{\Gamma}$ and $s=\left\{s^{-}, s^{+}\right\}, \tilde{s}=\left\{\tilde{s}^{-}, \tilde{s}^{+}\right\}$in the part $M_{\Gamma}$ and $\tilde{M}_{\tilde{\Gamma}}$ of the "upper" chamber $\Omega_{2}$ (and $\widetilde{\Omega}_{2}$ ) as well, see Notation 2.12. Let us reconstruct the eigenfunctions into that part of $\Omega_{2}$ (and $\widetilde{\Omega}_{2}$ ), thus $M_{\Gamma}$ is isometric to $\tilde{M}_{\tilde{\Gamma}}$ as eigenfunctions are equal in them and eigenfunctions determine metrics.

Now we are ready to continue the procedure of the Section 5.3 , replacing $\Gamma$ and $\tilde{\Gamma}$ by new equal parts of $\partial \Omega_{2}$ and $\partial \widetilde{\Omega}_{2}$ interfaces. 


\subsection{Polyhedra isometry}

Step by step comparing two admissible Riemannian polyhedra $\mathcal{M}$ and $\widetilde{\mathcal{M}}$, we came to the conclusion that all chambers' interiors are identical and the interfaces are identical too, thus we can show that $\mathcal{M}$ and $\widetilde{\mathcal{M}}$ are equivalent as simplicial complexes.

What is the guarantee that we have not missed any chamber or interface? Assume that we have missed some chamber on one of the polyhedra, say we have an extra chamber $\widetilde{\Omega}_{l}$ on $\widetilde{\mathcal{M}}$. "Extra" means that there is no isometric chamber on $\mathcal{M}$ to it after all our procedures. We should mention here, that as a simplicial complexes, our polyhedra are $n-1$ chainable, thus any chamber can be reached from any point through interfaces. Also, we recall, that there are just a finite number of chambers on the polyhedron. Consider any interface $\tilde{\gamma}$, that belongs to $\widetilde{\Omega}_{l}$, this interface should have another common chamber, say, $\widetilde{\Omega}_{l-1}$. If this $\widetilde{\Omega}_{l-1}$ has an isometric copy on $\widetilde{\mathcal{M}}$, thus we can use $\tilde{\gamma}$ for our constructions and see, that $\widetilde{\Omega}_{l}$ should be isometrical to some chamber on $\mathcal{M}$. If there is no isometrical chamber for $\widetilde{\Omega}_{l-1}$ as well, we can consider the next interface and continue the procedure we will conclude, that there are no isometrical chambers on $\mathcal{M}$ for all chambers on $\widetilde{\mathcal{M}}$ (as there are just finite number of them).

Assume now, that we have missed an interface, say on $\tilde{\gamma}_{m l} \subset \widetilde{\mathcal{M}}$ such that there is no isometrical interface on $\mathcal{M}$. By the definition of admissible Riemannian polyhedra, there are always chambers, that are adjacent to that interface, say $\widetilde{\Omega}_{m}$ and $\widetilde{\Omega}_{l}$. As we have shown, that all chambers on $\mathcal{M}$ and $\widetilde{\mathcal{M}}$ are isometric, thus there are chambers $\Omega_{m}$ and $\Omega_{l}$ isometric to $\widetilde{\Omega}_{m}$ and $\widetilde{\Omega}_{l}$ on $\mathcal{M}$. Thus the interface $\tilde{\gamma}_{m l}$ should be isometric to some interface, common for $\Omega_{m}$ and $\Omega_{l}$.

Thus we have proved that $\mathcal{M}$ and $\widetilde{\mathcal{M}}$ are equivalent as simplicial complexes.

On each step we have chosen the interface to cross arbitrarily as each chamber's 


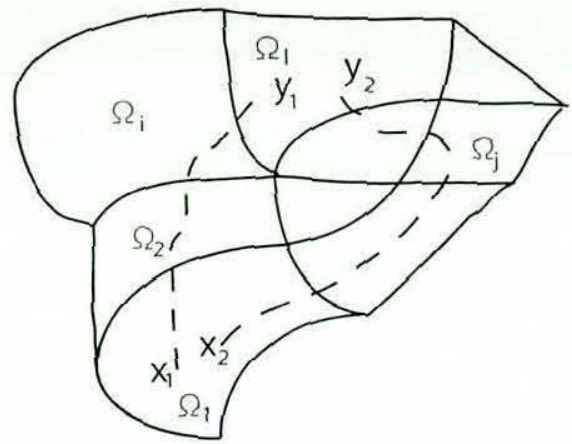

Figure 5.10: Two different paths.

boundary could consist of a finite number of interfaces. Thus we have to show now, that the choice of the path on our polyhedron would not affect on the identification of points and chambers. Consider any two possible paths $l_{1}$ and $l_{2}$ starting from $\mathrm{x} \in \Omega_{1}$ and finishing in, say $\mathrm{y} \in \Omega_{l}$, see Figure 5.10. How can we understand that they came to the same chamber? We can see that once we reached some chamber, that the eigenfunctions depend uniquely on the given boundary data. The eigenfunctions identify points on polyhedra. We compare eigenfunction values for points inside chamber and conclude, whether we are inside the same chamber (if there are the same eigenfunction values, thus identical points) or not.

We identify points on the polyhedra by the following procedure: We know that the eigenfunctions $\varphi_{k}$ of our problem distinguish points on polyhedron, i.e. if $\varphi_{k}\left(\mathrm{x}_{1}\right)=$ $\varphi_{k}\left(\mathrm{x}_{2}\right), k=1,2, \ldots$ then $\mathrm{x}_{1}=\mathrm{x}_{2}$. We also know that all eigenfunctions are identical for both admissible Riemannian polyhedra $\mathcal{M}$ and $\widetilde{\mathcal{M}}$, by our procedure. We use these properties to compare points on them.

We compare points on both polyhedra by the following rule (see subsection 4.2.3, or [38]). Let $\widetilde{\mathcal{N}}$ and $\widetilde{\mathcal{N}}^{\prime}$ be subsets of $\widetilde{\mathcal{M}}$. In the manifolds $\mathcal{M}_{\partial \tilde{\mathcal{N}}}$ and $\mathcal{M}_{\partial \widetilde{\mathcal{N}}^{\prime}}$ we say, that the points are equal if $\widetilde{\mathrm{x}} \in \mathcal{M}_{\partial \tilde{\mathcal{N}}}$ and $\widetilde{\mathrm{x}}^{\prime} \in \mathcal{M}_{\partial \widetilde{\mathcal{N}}^{\prime}}$, such that $\widetilde{\varphi}_{j}(\widetilde{\mathrm{x}})=\widetilde{\varphi}_{j}\left(\widetilde{\mathrm{x}^{\prime}}\right)$ for all $j=1,2, \ldots$ In this case, the points $\widetilde{\mathbf{x}}$ and $\widetilde{\mathbf{x}^{\prime}}$ correspond to the same point on $\widetilde{\mathcal{M}}$. As 
we have shown, there is a point $\mathrm{x}$ on $\mathcal{M}$ such that $\varphi_{j}(\mathrm{x})=\widetilde{\varphi}_{j}(\widetilde{\mathrm{x}})$, analogously, we identify points these points.

There is a one-to-one correspondence between the chambers and interfaces and portions of the boundary with their inner metrics. Thus $(\mathcal{M}, g)$ is isometric to $(\widetilde{\mathcal{M}}, \tilde{g})$, with equal distances as an approximations of minimizing curve (see (2.20)). Thus we have shown, that two polyhedra with equal BSD given on isometric parts of the boundaries are necessarily isometric. 
$\Gamma_{\text {Chapter }}$

\section{Conclusions and Outlook}

The following results were obtained in the thesis:

- Following Fuglede B. and Eells J., [25], we described the geometric structure of a class of admissible Riemannian polyhedra (ARP) and introduced a length and a geodesic spaces on it. We introduced a Laplace operator and considered its spectral properties on the admissible Riemannian polyhedron.

- Generalizing procedures by Babich V., Ulin V., [6] and Katchalov A., [36], we developed a theory of non-stationary Gaussian beams on the admissible Riemannian polyhedron. The main novelty is the description of the behavior of Gaussian beam which hits the interface. We proved anisotropic analogues of Snell's and Frenel's laws for the reflected and transmitted beams. These results were published in [41].

- We gave a solution to the inverse boundary spectral problem, namely we proved the uniqueness for this problem. We considered inverse boundary spectral problem for a Riemannian polyhedron under some mild geometric and analytical assumptions. We have proven that two Riemannian polyhedra having equal 
boundary spectral data on open parts of the boundary are isometric. This significantly generalizes results obtained earlier for the smooth inverse boundary spectral problem.

Let us define important open questions.

- Generalize the obtained result for the piece-wise smooth anisotropic Schrodinger operator, corresponding to the admissible Riemannian polyhedron. Next, obtain uniqueness theorem for the general elliptic second-order differential operator.

- Consider some more general structure of the Riemannian polyhedron without analytical and geometric restrictions, namely, allow the presence of artificial interfaces, allow the metric to be continuous having jumps in its derivatives.

- Obtain the reconstruction procedure for an admissible Riemannian polyhedron $\mathcal{M}$, for the potential of the Schrodinger operator $q$, and metric tensor $g$ from given boundary spectral problem on the open part of the boundary $\Gamma \subset \partial \mathcal{M}$.

- Consider the dynamical formulation of the problem. 


\section{Bibliography}

[1] M. Agranovich, Elliptic boundary problems, Encyclopedia Math. Sci., 79, Partial Differential Equations, IX, 1-144, Springer, Berlin, 1997.

[2] G. Alessandrini, V. Isakov, Analicity and uniqueness for the inverse conductivity problem, Rend.Istit.Mat.Univ.Trieste 28(1997), no.1-2, 351-369.

[3] N. Akhiezer, I. Glazman, Theory of Linear Operators in Hilbert Space. Dover Publications, Inc. New York, 1993.

[4] K. Astala, M. Lassas, L. Päivärinta, Calderon's inverse problem for anisotropic conductivity in the plane, Communications in Partial Differential Equations $30(2005)$, no. $1-3,207-224$.

[5] M. Anderson, A.Katsuda, Ya. Kurylev, M. Lassas, M. Taylor, Boundary Regularity for the Riccati equation, Geometry Convergence and Gelfand's Inverse Boundary Problem, Inventiones Mathematicae 158(2004), 261-321.

[6] V. Babich, V. Ulin, The complex space-time ray method and "quasiphotons", (Russian) Zap. Nauchn. Sem. LOMI, 117(1981), 5-12.

[7] V. Babich, V. Buldyrev, I. Molotkov, The space-time Ray Method, Linear and Nonlinear waves, (Russian), Leningrad Univ., Leningrad, 1985. 
[8] W. Ballman, A volume estimate for piecewise smooth metrics on simplicial complexes, Rendiconti Sem. Mat. Fis. Milano 66(1996), 323-331.

[9] M. Belishev, An approach to multidimensional inverse problems for the wave equation, (Russian) Dokl. Akad. Nauk SSSR 297(1987), no.3, 524-527; translated in Soviet Math. Dokl. 36(1988),no.3 481-484.

[10] M. Belishev, Wave basis in multidimensional inverse problems, (Russian) Mat.Sb. 180(1989), 584-602.

[11] M. Belishev, A. Blagoveschenski, Dynamical inverse problems of wave theory, Izd-vo S.-Pet. Universiteta, 1999.

[12] M. Belishev, A. Katchalov, A boundary control and quasiphotons in a problem of the reconstruction of a Riemannian manifold from dynamic data, (Russian) Zap.Nauchn. Sem. POMI 203(1992), 21-50; translated in J.Math.Sci. 79(1996), no.4, 1172-1190.

[13] M. Belishev, Ya. Kurylev, To the construction of a Riemannian manifold via its spectral data (BC-method), Communications in Partial Differential Equations 17(1992), no.5-6, 591-594.

[14] M. Birman, M. Solomyak, Spectral theory of self-adjoint operators in Hilbert space, D.Reidel Publishing, Dordrecht, 1987.

[15] A. Blagoveschenski, A one-dimensional inverse boundary value problem for a second order hyperbolic equation, (Russian) Zap. Nauchn. sem. LOMI, 15(1971), 85-90.

[16] A. Bogoljubov, V. Kravtsov, Problems on Mathematical Phisics, (Russian), Izdatelstvo Moskovskogo Universiteta, 1998.

[17] D. Burago, Yu. Burago, S. Ivanov S, A Course of Metric Geometry, AMS, Graduate Studies in Mathematics, Volume 33, 1998. 
[18] Yu. Burago, V. Zalgaller, Geometry III Theory of Surfaces Series: Encyclopaedia of Mathematical Sciences, 48(1992).

[19] G. De Cecco, G. Palmeri, On the reqularity of eigenfunctions of the Laplace operator on a Lipschitz manifold, Journal of Math. Pures Appl., (9), 68(1989), no.1, 121-134.

[20] G. De Cecco, G. Palmeri, Harmonic forms on compact Lipschitz manifolds, Boll.Un.Mat.Ital. A(7), 2(1988), no.1, 101-108.

[21] G. De Cecco, G. Palmeri, Length of curves on Lip manifolds, Atti Accad.Naz.Lincei Cl. Sci.Fis.Mat.Natur.Rend.Licei (9) Mat.Appl, 1(1990), no.3, 215-221.

[22] G. De Cecco, G. Palmeri, Intrinsic distance on a Lipschitz Riemannian manifold, rend. Sem.Mat.Univ.Politec.Torino, 46(1988), no.2, 157-170; Distanza intrinseca su una varieta Riemanniana di Lipschitz, Rend.sem.Mat.Univ.Torino, 46(1988), 157-170.

[23] A. Connel, Modern Differential Geometry for Physicists, Mac.Application of Tensor Analysis, (in Russian), Gos. Izd-vo fiz-mat literatury, 1963.

[24] A. Edmonds, R. Fintushel, Singular circle fiberings, Math. Z. 151(1976), 89-99.

[25] J. Eells, B. Fuglede, Harmonic maps between Riemannian polyhedra. With a preface of Gromov M., Cambridge Tracts in Mathematics, 142, Cambridge University Press, Cambridge, 2001.

[26] Y. Egorov, V. Kondratiev, On Spectral Theory of Elliptic Operators, Birkhauser Verlag, 1996.

[27] L. Evans, Partial Differential Equations, AMS, Graduate Studies in Mathematics, Volume 19, 1998. 
[28] H. Federer, Geometric Measure Theory, Springer-Verlag, Berlin, Heidelberg, New York, 1996.

[29] B. Fuglede, Finite energy maps from Riemannian polyhedra to metric spaces, Ann.Acad.Sci.Fenn.Math., 28(2003), no.2, 433-458.

[30] B. Fuglede, Holder continuity of harmonic maps from Riemannian polyhedra to spaces of upper bounded curvature, Cal. Var., 2002.

[31] I. Gelfand, B. Levitan, On the determination of a differential equation from its spectral function, Iz.Akad.Nauk SSSR, Ser.Mat. 15(1951), 309-360.

[32] L. Hörmander, The analysis of linear partial Differential Operators, Vol. I-IV. Springer-Verlag, Berlin, 1985.

[33] L. Hörmander, Linear partial differential operators, Die Grundlehren der mathematischen Wissenschaften, 116, Springer-Verlag, Berlin-Gottingen-Heidelberg, 1963.

[34] L. Hörmander, A uniqueness theorem for second order hyperbolic differential equations, Comm. Part. Diff. Equations 17(1992), no. 5-6, 699-714.

[35] V. Isakov, On uniqueness of recovery of a discontinuous conductivity coefficient, Comm.Pure Appl.Math., 41(1988), no.7, 865-877.

[36] A. Kachalov, Gaussian beams, Hamilton-Jacobi equations and Finsler geometry, Zapiski Nauchn. Semin.POMI, 297(2003), 66-92.

[37] A. Kachalov, A system of coordinates for describing the quasiphoton", Zap. Nauchn. Sem LOMI, 140(1984), 73-76.

[38] A. Kachalov, Ya. Kurylev, M. Lassas, Inverse Boundary Spectral Problems, Chapman Hall / CRC 123, 2001. 
[39] Katchalov A., Kurylev Ya., Lassas M., Mandache N., Equivalence of time-domain inv.prob. and bound.spectral problem, Inv.prob. 20(2004), 419-436.

[40] M. Kervaire, A manifold which does not admit any differentiable structure, New York (USA), Commentarii mathematici Helvetici, 34(1960), 257-270.

[41] A. Kirpichnikova, Propagation of a Gaussian beam near an interface in an anisotropic medium, Zapiski Nauchn. Sem. POMI, 324(34)(2005), 77-109.

[42] A. Kirsch, L. Päivärinta, On recovering obstacles inside inhomogeneities, Math.Meth.Appl.Sci., 21(1998), 619-651.

[43] H. Koch, D. Tataru, Carleman estimates and unique continuation for the second order elliptic equations with nonsmooth coefficients, 2000.

[44] M. Krein, Solution of the inverse Sturm-Liouville problem, Doklady Akad.Nauk SSSR, 76(1951), 21-24.

[45] Ya. Kurylev, On the Holmgren-John Uniqueness theorem, Zap.Nauchn.Sem.LOMI, 203(1992).

[46] O. Ladyzhenskaja, The Boundary Problems of Mathematical Physics, (in Russian), Izd. Nauka, Glavnaja redakcija Fiz-mat literatury, Moskva, 1973. Also: Appl. Math. Sciences 49, 1985, Springer-Verlag.

[47] M. Popov, Ray Theory and Gaussian Beam Method for Geophysicists, Edufba, Salvator-Bahia, 2002.

[48] J. Ralston, Gaussian beams and propagation of singularities, Studies in PDE, MAA Studies in Mathematics, 23, Walter Littman ed, 1983.

[49] L. Robbiano, C. Zhuily, Uniqueness in the Cauchy problem for operators with partially holomorphic coefficients, Invent. Math., 131(1998), no.3, 493-539. 
[50] S. Sobolev, Equations of Mathematical Physics, (in Russian), Moskva, Izd-vo Nauka, Glavnaja redakcija fiz-mat literatury, 1966.

[51] H. Seifert, W. Threlfall, Lehrbuch der Topologie, Chelsea Publishing Co., New York, 1945.

[52] E. Stein, Topics in harmonic analysis related to the Littlewood-Raley theory, Princeton Univ. Press, Princeton, N.Y., 1970

[53] K. Sturm, On the $L^{p}$-spectrum of uniformly elliptic operators on Riemannian manifolds, J.Func.Anal., 118(1993), no.2, 442-453.

[54] K. Sturm, Metric spaces of lower bounded curvature. Exposition Math., 17(1999), no.1, 35-47.

[55] K. Sturm, The geometric aspects of Dirichlet forms, New directions in Dirichlet forms, AMS/IP Srud.Adv.Math., 8(1998), Amer.Math.Soc., Providence, RI, 233277.

[56] D. Tataru, Unique continuation for solutions to PDE: between Hormander's theorem and Holmgren's theorem, Comm. PDE, 20(1995), 855-884.

[57] D. Tataru, Boundary observability and controllability for evolutions governed by higher order PDE, J. Math. Anal. Appl., 193(1995), no. 2, 632-658.

[58] D. Tataru, Unique continuation for operators with partially analytic coefficients, J. Math. Pures Appl. (9), 78(1999), no.5, 505-521.

[59] M. Taylor, Tools for PDE, AMS, Providence, R.I., 2000.

[60] M. Taylor, Partial Differential Equations, Vols. 1-3, Springer-Verlag, New York, 1996.

[61] H. Triebel, Function Spaces, Birkhauser, Boston, 1983.

[62] V. Zorich, Mathematical Analysis I, II, Series: Universitext, Springer, 2004. 